- Dual-Active-Bridge Isolated DC-DC Converter With Variable Inductor for Wide Load Range Operation

\author{
Sarah Saeed ${ }^{\circledR}$, Student Member, IEEE, Jorge Garcia ${ }^{\circledR}$, Senior Member, IEEE, \\ and Ramy Georgious (-) Member, IEEE
}

\begin{abstract}
This article explores the use of a variable inductor as a reactive element for energy transfer in a dual-active-bridge (DAB) converter. By using a controlled variable inductor, the optimal switching region in the operation of the phase-shift DAB can be extended, and thus, high efficiencies can be achieved over wider load ranges compared to the traditional solution. Moreover, the combined use of the variable inductance together with the phase shift, as two control parameters, allows for the linearization of the DAB converter transfer function, which improves the stability and the performance of the controller. And finally, due to the controlled saturation of the device, it is feasible to reduce the magnetic core size, yielding a size optimization of the full converter. This article develops a circuit-based model to simulate the converter system, aiming to study the proposed improvements. Furthermore, a 2-kW SiC-based DAB converter prototype is constructed, including a controlled variable inductor. The experimental results presented in this article validate the studies carried out for the different operation conditions.
\end{abstract}

Index Terms-Dual-active-bridge, efficiency, hysteresis losses, variable magnetics, zero voltage switching.

\section{$\mathbf{T}$} HE electrical utility network of today is facing a significant change driven by the need for improved efficiency, reliability, and safety. To achieve these requirements, there is a growing interest toward the smart grid systems which incorporate different systems using power electronics technologies. The most important of these systems is the solid-state transformer (SST)

Manuscript received October 12, 2020; accepted December 18, 2020. This work was supported in part by the European Union's H2020 Research and Innovation Programme under Grant 864459 (UE-19-TALENT-864459), in part by the Spanish Government (Innovation Development and Research Office-MEC) under Research Grant ENE2016-77919, "Conciliator" Project, "B2B" Project Nuevas vías hacia la gestión descentralizada de la energía de edificio a edificio PID2019-111051RB-100, and “Torres Quevedo" Grant PTQ2019-010579, and in part by the Government of the Principality of Asturias under Grant FC-GRUPIN-IDI/2018/000241, and in part by the "Severo Ochoa" Program of Predoctoral Grants for Training in Research and University Teaching under Grants PA-17-PF-BP16-133 and PA-13-PF-BP13-138. Recommended for publication by Associate Editor R. Ayyanar. (Corresponding author: Sarah Saeed.)

Sarah Saeed and Jorge Garcia are with the Lemur group, Electrical Engineering Department, University of Oviedo, 33204 Gijon, Spain (e-mail: saeedsarah@uniovi.es; garciajorge@uniovi.es).

Ramy Georgious is with the Lemur group, Electrical Engineering Department, University of Oviedo, 33203 Gijon, Spain, and also with the Electrical Engineering Department, Port Said University, Port Said 42526, Egypt (e-mail: eng.ramy.georgious@hotmail.com).

Color versions of one or more figures in this article are available at https: //doi.org/10.1109/TPEL.2020.3048928.

Digital Object Identifier 10.1109/TPEL.2020.3048928 which can radically change the utility power distribution by more efficient routing of power, voltage regulation, and flexibility. The basic and most popular structure of the SST is the dual-active bridge (DAB). DAB converter allows bidirectional power flow by means of regulating the phase shift (PS) between the converter bridges. However, this modulation scheme can achieve high efficiencies only at voltage levels that match the transformer turns ratio; significant mismatches lead to transformer RMS currents, inductor and transformer copper losses, and increased semiconductor conduction losses [1]-[3]. Also, circulating currents take place due to the PS between the voltage and the current applied to the transformer [4]. Another limitation is the loss of zero-voltage switching (ZVS) at light loading conditions [5], [6]. For tackling those limitations, many alternative modulation schemes have been proposed in [3], [4], and [6]-[8]. As the complexity of the modulation scheme increases, it compromises the ease and time of implementation of the control function [9].

Another approach to tackle the limitations of the DAB operation is to use the variation in the switching frequency as an alternative parameter to control the power flow in the converter. However, the frequency variation has a significant effect on the converter efficiency specifically in the presence of energy storage systems (ESS) [10], [11]. The switching frequency variation range is also limited by the design of magnetic components, namely, the series inductor and the high-frequency (HF) transformer. Finally, it is not straightforward to change the frequency online during the converter operation since it implies a change in the sampling period of the programmer, as well as the interrupt periods for acquiring correct measurements of the sensors data. Consequently, to widen the soft-switching range and reduce circulating currents, most of the literature works have explored modulation strategies which maintained a fixed switching frequency [12]. To this motivation, the inductance variation, achieved throughout the use of variable inductors (VI), is proposed in this work as a promising control parameter of the power flow in the DAB converter [13].

VI is an electromagnetic device that is based on changing the inductive impedance of a winding placed around a saturable magnetic core [14]. This mechanism is achieved by changing the saturation level of the magnetic core [14]. These devices, therefore, allow for additional degrees of freedom in the design and control of power electronic converters (PECs) [14]. This is particularly useful in resonant converters, where the output power is generally controlled by varying the switching frequency due to the power versus frequency characteristic of 
the converter [15], [16]. This standard frequency control presents some drawbacks due to electromagnetic interference (EMI) issues, complex filtering, or variable sampling time, among others, especially for a wide range of variation. However, if variable magnetics are used, the same control margins can be obtained at a constant switching frequency, therefore, allowing for an optimization of the EMI filters and sampling procedures [14], [17].

Also for $\mathrm{dc}-\mathrm{dc}$ bridge converters, such as the DAB or the dual-half-bridge (DHB), the addition of a new degree of freedom to the control scheme by means of the VI can enhance the operational performance of the system, for instance, by increasing the soft-switching margins, as reported in preliminary works [1], [2]. Specifically, in [1], where the VI concept was proposed as the main energy transfer element for DHB converter, the study concluded that the ZVS operation region of such topologies is controlled by means of PS control by employing an adaptive inductor. Therefore, high efficiency can be achieved over wider load ranges compared to the conventional converter. On the other hand, for the DAB converter, the authors in [2] adopted a solution based on inserting an additional series inductor at light load conditions, aiming to decrease circulating currents and thus, allowing for an increase in the converter efficiency. Consequently, these studies reinforce the idea of introducing an adaptive power transfer inductor in the DAB converter to reduce the overall power losses. However, the solution proposed in [2] is based on mechanical switching of a combination of fixed inductance values. The obvious benefits of mechanical switching are the simplicity and low cost. However, it allows only stepwise control, and it is too slow to provide a stable operation. On the other hand, the magnetically controlled VI allows continuous variation of the inductance; therefore, it will be used in the proposed study herein.

The improvement of the DAB converter efficiency is not the only feature that the VI can provide, there are several potential features that can be explored. Another feature is the use of the inductance variation to linearize the control-to-output transfer function. This has implications in the static gain characteristic, which can allow several advantages to the converter control system. For example, in solar converters, the maximum power point tracking (MPPT) technique can be smoother without introducing dithering or instability due to nonlinear power flow characteristics.

Another potential improvement that the VI can add to the DAB converter is the possibility to extend the maximum power level, which results from decreasing the inductance as a function of the PS to allow linear phase-to-power transfer function. This implies that, by means of controlled saturation of the magnetic core, the same magnetic core size can be used for a higher power level. This could allow optimizing the size of the magnetic element. However, the possibility to boost the converter power density by using VI is limited by the additional volume and losses of the power electronic devices which are needed for controlling the magnetic core saturation [18].

Moreover, it is worth to notice that replacing the fixed power transfer inductance by a variable one, the dynamic performance of the control scheme can also be modified. In fact, it is

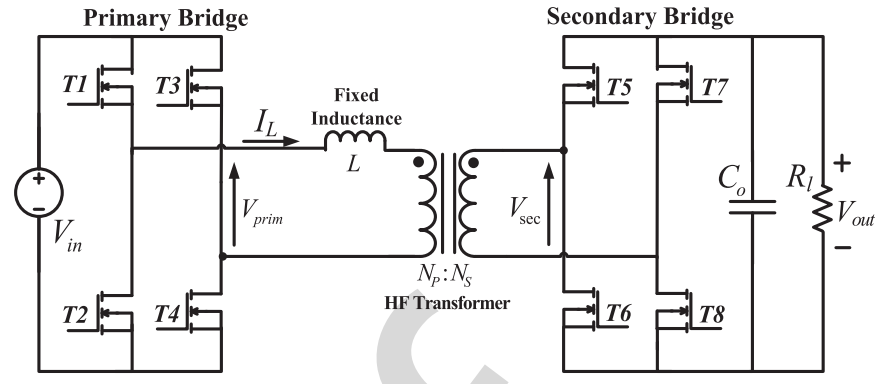

Fig. 1. Schematic of DAB converter

postulated that regarding the disturbance rejection upon closedloop control of the converter, the adaptive inductor adds an extra control parameter, which can be employed to assist the main control scheme in riding through the transient event. In all cases, it is necessary to investigate the overall PEC figures of merit incorporating the VI.

The aim of this article is to study the potential enhancements to the operation of the DAB converter by using a VI. The focus of this article is to present the improvements in the converter efficiency under light loads (by extending the ZVS range) and heavy loads (by reducing circulating currents), as well as the linearization of the power flow characteristics, and finally, the improvement of the dynamic response of the controlled system.

The analysis is carried out using an electromagnetic system simulation model which takes into consideration different component losses, such as switching and conduction losses, magnetic device core and winding losses, etc. In this context, Section II states the theoretical background that discusses the structure and the principle of operation of the conventional DAB converter. Also, the latter section provides a briefing about the VI structure, operation, and model. Section III explains the models for different system devices. The models are adjusted with the electrical characteristics of the test setup, in order to develop real simulations and particularize the analysis for the constructed prototype. In Section IV, several potential aspects are studied for extending the operation range of the DAB converter by using a VI. In Section V, the test setup and the experimental results are presented. Finally, Section VI concludes the article.

\section{Proposed DAB Using VI}

\section{A. Principle of Operation of Conventional DAB Converter}

The DAB converter is a bidirectional isolated dc-dc power conversion topology which is composed of two full-bridge inverter circuits, interfaced across a constant power transfer inductor that usually includes an HF transformer, as clarified by Fig. 1 .

The flexibility of the primary and secondary active full bridges in this topology allows for various modulation methods to minimize the losses and to maximize the efficiency over a wide load and voltage operation ranges [6], [19]. Using the simplest modulation technique, named single-PS (SPS) scheme, the full-bridge converters are operated at a constant switching frequency, with 50\% maximum duty cycle. After a thorough 


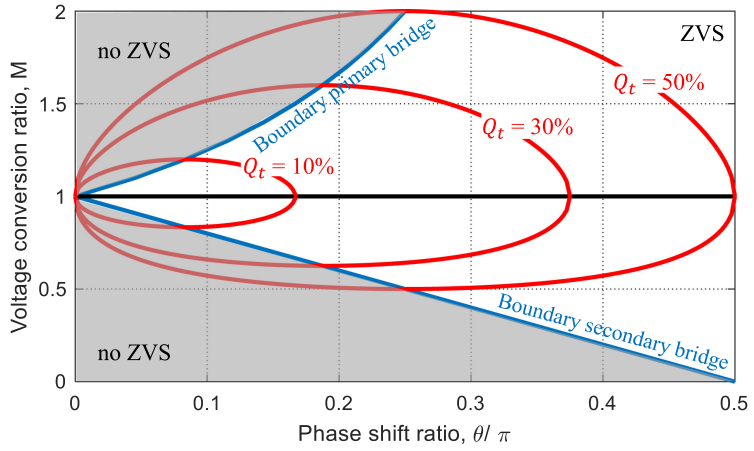

Fig. 2. ZVS boundaries of the DAB converter.

study of the circuit operation, it can be demonstrated that the bridge leading by a phase angle sends power to the lagging one [20]. The analytical expression for the power flow in the converter under SPS modulation scheme is

$$
P_{\mathrm{DAB}}=\frac{V_{\text {in }} V_{\text {out }}}{X_{L} r_{t}} \theta\left(1-\frac{\theta}{\pi}\right) .
$$

From (1), the control parameters that affect the power transfer are the input and output voltage levels, $V_{\text {in }}$ and $V_{\text {out }}$, respectively, the transformer turns ratio $r_{t}=N_{s} / N_{p}$, the PS between the primary and secondary bridges $\theta$, and the impedance $X_{L}=2 \pi f L$, $f$ being the switching frequency, and $L$ the inductance of the coil.

In case of having a constant voltage source at the output of the DAB converter, then the wide-voltage and wide-load are distinctive terms that need to be handled carefully. The voltage range indicates the relationship between the input and output voltage levels, while the loading condition indicates the amount of power demanded either by the input or the output sources, i.e., in forward or reverse direction. On the other hand, in the case of having a resistive load at the output, the resistance value determines the voltage level for a certain amount of demanded power, in this case, only to the resistive load, i.e., in the forward direction. In this latter case, the signification of the wide-voltage and wide-load terms is the same and can be interchangeable.

\section{B. Limitations of the Converter Operation Under Wide-Voltage and Wide-Load Ranges}

To achieve ZVS in the converter switching devices, the inductor current should be enough to charge and discharge the parasitic output capacitors of the switches [21]. Analyzing the inductor current waveform in analogy with the switching waveforms at both bridges, the ZVS conditions for the primary and the secondary bridges can be formulated in terms of the voltage conversion ratio $M$ and the PS ratio $\theta / \pi$ as explained in detail in [13], [20], and [21]. The ZVS boundaries of the DAB converter can thus be represented as shown in Fig. 2.

It can be observed that, ideally, for $M=1$, ZVS can be achieved over the full range of operation. For values of $M$ different than 1, the PS must be large enough to provide the required energy for achieving the ZVS condition. Operation under small PSs, i.e., upon low load conditions, is thus limited by the ZVS range, which implies that the converter should be designed to operate at the maximum PS in order to allow higher values of current and facilitate ZVS.

On the other hand, higher values of PS imply that the phase angle between the current and the voltage applied to one side of the HF transformer increases as well. Consequently, the reactive circulating currents are higher. Those currents result in an increase in the conduction losses and elevate the reactive power losses [4], [22] which eventually cause a drop in the converter efficiency. To emphasize the effect of the PS on the reactive currents, the total percentage reactive currents in the converter is formulated again in terms of $M$ and $\theta / \pi$, as explained in detail in [21].

It can be concluded that under mismatches in the voltage levels $(M \neq 1)$, the soft-switching range is significantly constrained, which limits the operation of the converter at low PS angles. Additionally, since the PS angle between the bridges is the only control parameter, therefore, under higher PS angles, the reactive currents are higher.

Consequently, to widen the ZVS range and reduce circulating currents, most of the literature has explored new modulation strategies other than the SPS. Those alternative modulation schemes consist in introducing extra degrees of freedom to control the power transfer. This is achieved by inserting an extra PS angle between the modulation pulses of two legs in a full bridge [3], [4], [6]-[8]. Therefore, the resulting ac voltages will be three-level with a zero-voltage period during the switching transitions, which allows increasing ZVS and reducing circulating currents. However, the increase in the number of variables, introduced by these modulation schemes, results in cumbersome and time-consuming computations which might restrict the resources of digital processors in real-time programming [9].

This article hereafter proposes a different solution instead of changing the operational margins, that is, to change the operation point of the converter itself. This can be carried out by introducing another control parameter, which is the inductance variation in combination with the PS angle. From (1), it is clear that replacing the fixed inductor by a variable one can serve as an extra control parameter for the power transfer in the converter.

\section{VI: Structure and Operation}

From the study of the state of the art regarding possible physical structures to implement the VI, the double E-core setup, depicted in Fig. 3(a), is considered to be the most appropriate and most comprehend in the literature [23]-[25]. The structure consists of two E-cores stacked on top of each other to form three magnetic arms with an air gap in the center arm. The main winding is wound around the center arm, and the control windings are wound around the lateral left and right arms; they are serially connected in opposite polarity so as to cancel out the ac voltages induced by the center leg [25].

In this arrangement, the current flowing through the main winding $N_{c}$ generates an ac flux $\phi_{C}$ which circulates through the center arm and splits to the outer left and right arms. Applying a relatively small dc current $I_{b}$ to the bias control windings $N_{b}$, a dc flux is produced in the right $\operatorname{arm} \phi_{R}$ and the left $\operatorname{arm} \phi_{L}$, which tends to circulate mainly through the outer, ungapped, 


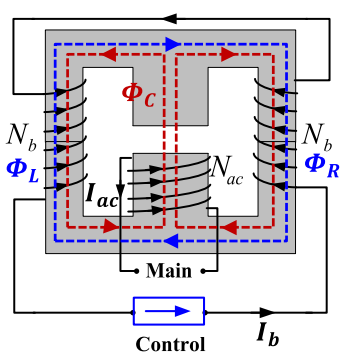

(a)

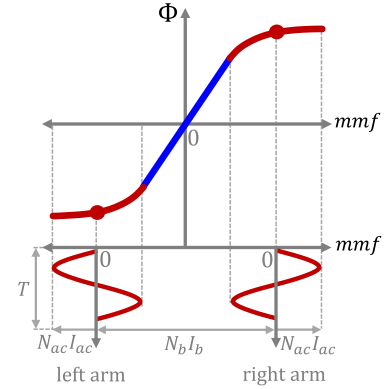

(b)

Fig. 3. Variable inductor based on double E-core structure. (a) Structure of the device and windings. (b) Operation of VI in nonlinear region of $B(H)$ curve.

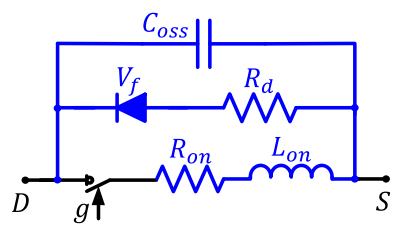

Fig. 4. SiC MOSFET model.

circumference of the core [25]. The dc flux will bias the operation point of the magnetic material within the $B(H)$ curve toward the nonlinear region, causing the inductance seen from the main winding terminals to vary as a function of the dc bias current [23], [26]. The operation is clarified by Fig. 3(b), which shows the operation points on the $B(H)$ curve for the left and right arms. The constant dc control current will produce a constant $m m f$ in the left and right arms, which will bring these sections into the nonlinear region of the magnetization curve.

\section{Electromagnetic System Model}

The following paragraphs aim to model the full system, which includes the DAB converter, the VI, and the HF transformer. Also, specific details and electrical characteristics of the devices used in the constructed prototype are added to the model. This will allow an accurate representation of the full system in order to analyze the performance of the converter when the conventional inductor is replaced by a variable one.

\section{A. Power Switches Model}

To account for the switching and conduction losses, the $\mathrm{SiC}$ MOSFETS are modeled as shown by the circuit diagram in Fig. 4. Specific to this application, the SCT2280KE devices are used [27], with the characteristic parameters indicated in Table I.

\section{B. HF Transformer Model}

The transformer used for the study is an HF planar transformer. The specifications and ratings of the transformer are stated in Table I. To characterize the transformer, the classic transformer circuit is used. The measurements and characterization were done as part of previous works [22], [28]. Nevertheless, a summary of the procedure and the obtained parameters are presented hereafter for convenience.
TABLE I

SPECIFICATIONS OF DIFFERENT DEVICES

\begin{tabular}{|c|c|c|}
\hline \multirow{6}{*}{$\begin{array}{l}\text { Power } \\
\text { Switch }\end{array}$} & Commercial Reference & SCT2280KE [27] \\
\hline & ON Resistance, $R_{o n}$ & $280 \mathrm{~m} \Omega$ \\
\hline & ON Inductance, $L_{o n}$ & $2.5 \mu \mathrm{H}$ \\
\hline & Diode Resistor, $\boldsymbol{R}_{d}$ & $300 \mathrm{~m} \Omega$ \\
\hline & Forward Voltage, $V_{f}$ & $4 \mathrm{~V}$ \\
\hline & Output Capacitance, $C_{o s s}$ & $27 \mathrm{pF}$ \\
\hline \multirow{10}{*}{$\begin{array}{c}\text { HF } \\
\text { Transformer }\end{array}$} & Commercial Reference & Himag planar 500 \\
\hline & Rated power & $10 \mathrm{~kW}$ \\
\hline & Transformer Ratio, $N_{p} / N_{s}$ & $18: 22$ \\
\hline & Primary Series Resistance, $\boldsymbol{R}_{s p}$ & $0.2 \Omega$ \\
\hline & Primary Series Inductance, $L_{s p}$ & $2.5 \mu \mathrm{H}$ \\
\hline & Secondary Series Resistance, $\boldsymbol{R}_{s s}$ & $0.2 /(22 / 18)^{2} \Omega$ \\
\hline & Secondary Series Inductance, $\boldsymbol{L}_{s s}$ & $2.5 /(22 / 18)^{2} \mu \mathrm{H}$ \\
\hline & Magnetizing Inductance, $L_{m}$ & $7.044 \mathrm{mH}$ \\
\hline & Magnetizing Resistance, $\boldsymbol{R}_{m}$ & $188.2 \mathrm{k} \Omega$ \\
\hline & Magnetizing Capacitance, $C_{m}$ & $0.2388 \mathrm{nF}$ \\
\hline \multirow{5}{*}{$\begin{array}{l}\text { Variable } \\
\text { Inductor }\end{array}$} & Magnetic Core Size & ETD49/25/16 \\
\hline & Magnetic Core Material & N87 \\
\hline & Main winding no. of Turns, $N_{c}$ & 23 turns \\
\hline & Bias winding no. of Turns, $N_{b}$ & 55 turns \\
\hline & Inductance Range & $70-140 \mu \mathrm{H}$ \\
\hline
\end{tabular}

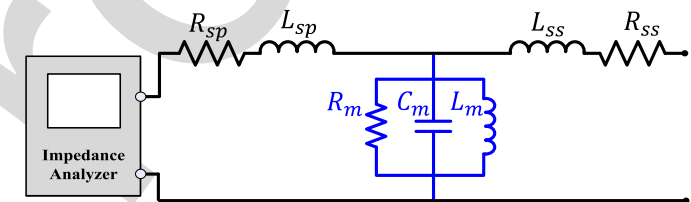

Fig. 5. No-load test to estimate magnetizing impedances of the transformer.

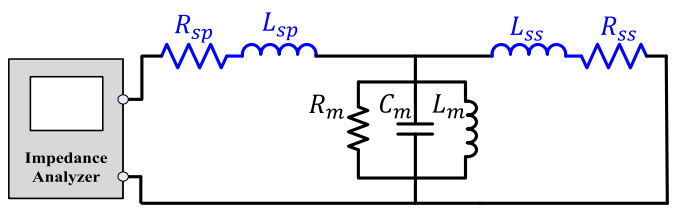

Fig. 6. Short-circuit test to estimate the series impedances of the transformer

To determine the characteristic parameters of the transformer, the conventional method is used which consists in performing open and short-circuit tests on the transformer windings. During the open-circuit test, also referred to as the no-load test, the impedance analyzer is connected to the terminals of the primary winding with the secondary winding open-circuited. Fig. 5 explains the process. The objective of this test is to estimate the magnetizing impedance of the transformer. Table I states the values of the obtained parameters. On the other hand, the short-circuit test was carried out by connecting the impedance analyzer to the primary winding with the secondary winding terminals short-circuited, as shown in Fig. 6. The objective of this test is to estimate the series inductance and resistance of primary and secondary windings. It is worth to note that the magnetizing impedance is much higher than that of the leakage path; therefore, the parallel magnetization branch can be neglected in the calculation. Table I states the values of the obtained parameters. Also, Fig. 7 illustrates the transformer circuit model along with the Bode plots illustrated in Fig. 8 . 


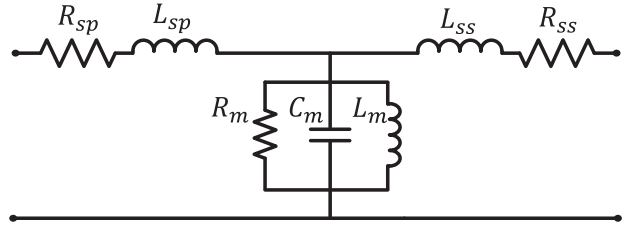

Fig. 7. HF transformer impedance model.

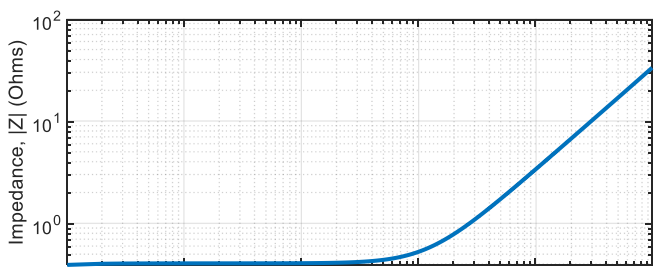

(a)

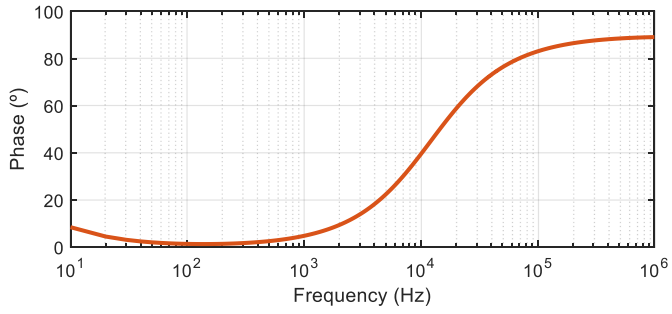

(b)

Fig. 8. Bode plots of transformer impedance model illustrating the (a) amplitude and (b) angle.

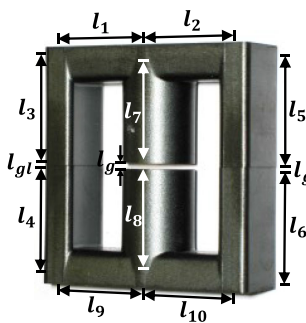

(a)

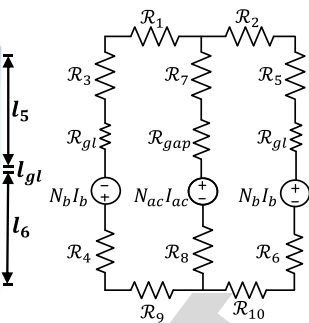

(b)

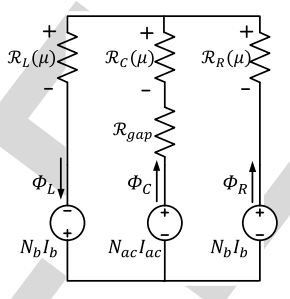

(c)

Fig. 9. Reluctance circuit of double E-core VI shown in Fig. 3. (a) Lengths of reluctance paths. (b) Corresponding circuit model. (c) Reluctance equivalent circuit.

\section{VI Model}

The VI is modeled based on the reluctance equivalent circuit [23], [24]. The magnetic core is modeled using three main elements. First, the constant reluctance, which models the nonferromagnetic material, such as the air gap, $\mathcal{R}_{\text {gap }}$. Second, the variable reluctance, which models the nonlinear behavior of ferromagnetic material, such as the left, right, and center arms, represented by $\mathcal{R}_{C}(\mu), \mathcal{R}_{L}(\mu)$, and $\mathcal{R}_{R}(\mu)$, respectively. And finally, the magnetomotive source, which models the winding electrical and magnetic interaction, such as the control windings represented by the duplicated sources $N_{b} . I_{b}$, and the main winding represented by $N_{c} . I_{c}$. The equivalent reluctance circuit model corresponding to the double-E core VI is illustrated in Fig. 9.

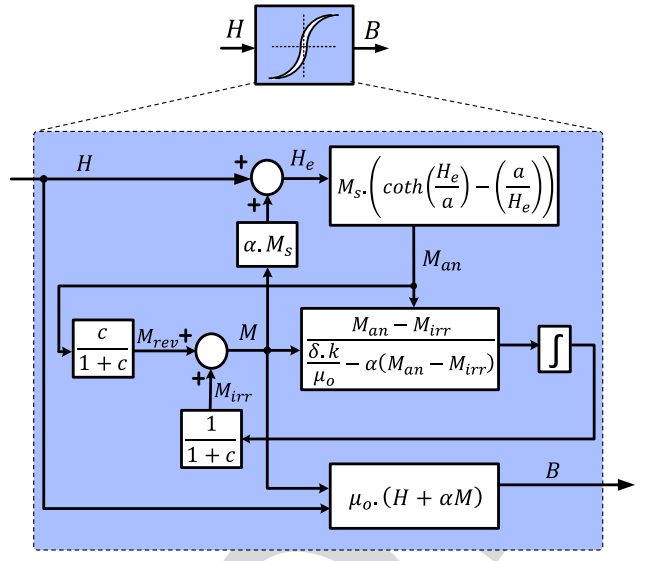

Fig. 10. Schematic of the implementation of JA model.

Therefore, the inductance can be calculated based on the reluctances of different paths in the magnetic circuit, as shown by

$$
L=\frac{N_{\mathrm{ac}}{ }^{2}}{\mathcal{R}_{C}(\mu)+\mathcal{R}_{\text {gap }}+\mathcal{R}_{L}(\mu) / / \mathcal{R}_{R}(\mu)} .
$$

The fully developed model is described in detail in previous works by the authors [24], [26]. The latter model takes into consideration a detailed description and modeling of the device losses, mainly core losses and winding eddy current losses. The necessary highlights of this loss model are presented below.

1) Model of Core Losses: The core losses in a magnetic device are divided into hysteresis loss and eddy currents loss. Previous studies in the literature [21] have shown that for a ferrite magnetic material operating in a range of frequency up to $100 \mathrm{kHz}$, the eddy current losses are a very small part of the total core losses. Therefore, for the range of frequencies under study herein, the eddy current losses in the magnetic core will be neglected for simplicity.

To calculate the hysteresis losses, the Jiles-Atherton (JA) hysteresis model is used [31]. The main strengths of the JA model compared to its counterpart models are — being the most suitable for development from a circuital simulation perspective, besides having good convergence, and acceptable accuracy among a variety of materials and operation conditions [33].

Fig. 10 shows a block diagram of the detailed implementation of the JA equations [26]. The parameters of the model are mainly five: $M_{s}, a, k, c$, and $\alpha$, which are initially estimated by an iterative procedure to fit the model to the magnetic material $B(H)$ curve data provided by the manufacturer [26]. Following this block diagram, the model equations have been implemented in Simulink. Therefore, for a given core size and magnetic material, the instantaneous magnetic flux density $(B)$ can be estimated for a certain instantaneous magnetic field intensity $(H)$ applied to the magnetic core.

2) Model of Winding Losses: The winding losses in a magnetic component are due to the resistance of the copper wire. At dc operation, or current waveforms of relatively low frequencies, this resistive component $R_{\mathrm{dc}}$ can be considered constant, and is a function of the resistivity of copper material, the temperature and 


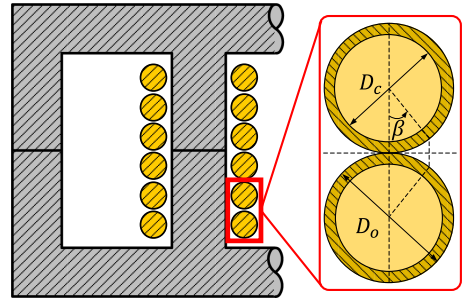

(a)

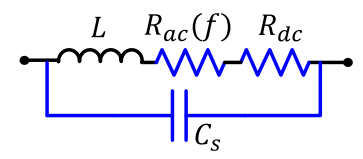

(b)

Fig. 11. Variable inductor winding model. (a) Configuration of the windings. (b) Full circuit model.

the geometry of the wire. However, as the switching frequency increases, two effects start to appear, which affects the resistance, the skin effect and proximity effect, both caused by eddy currents induced in the windings. These two effects alter the resistance of the winding and significantly contribute to the overall winding losses. It is necessary in this case to calculate the ac resistance of the winding.

Dowell provided a method that computes the equivalent winding resistance using a 1-D analytical approach [34]. It estimates the ac resistance $R_{\mathrm{ac}}$ by scaling the dc winding resistance $R_{\mathrm{dc}}$ by a factor

$$
R_{\mathrm{ac}}=R_{\mathrm{dc}}\left(M^{\prime}+\frac{\left(m^{2}-1\right) D^{\prime}}{3}\right)
$$

where $M^{\prime}$ and $D^{\prime}$ are coefficients defined based on the geometrical dimensions of the winding, material characteristics, and frequency of operation, and $m$ is the number of layers.

In order to attain a full, comprehensive model of the device, the stray capacitance of the winding is added to the model The calculation of the stray capacitance is based on an analytical approach that divides the winding into partitions [35]. It considers turn-to-turn and turn-to-core capacitances of the inductor. The overall total stray capacitance of the coil converges to the expression: $C_{s} \cong 1.366 C_{t t}$, where $C_{t t}$ is the turn-to-turn capacitance of the coil, defined by

$$
C_{t t}=\varepsilon_{0} l_{t}\left[\frac{\varepsilon_{r} \beta^{*}}{\ln \left(D_{o} / D_{c}\right)}+\cot \left(\frac{\beta^{*}}{2}\right)-\cot \left(\frac{\pi}{12}\right)\right]
$$

where $l_{t}$ is the turn length, $\beta^{*}$ is the angular coordinate, $\varepsilon_{0}$ and $\varepsilon_{r}$ are the permittivity of air and relative permittivity of the insulation medium, respectively, and $D_{0}$ and $D_{C}$ are the diameters of the wire with and without the insulation coating, respectively. The VI winding configuration is illustrated in Fig. 11(a).

Also, the full impedance model of the winding in the magnetic device is expressed by the circuit diagram in Fig. 11(b).

It is worth to note that the design of a VI for handling an ac high-frequency main current, as the case of the DAB converter in context, is different in two aspects specifically; first, the winding losses in the device will be different. The absence

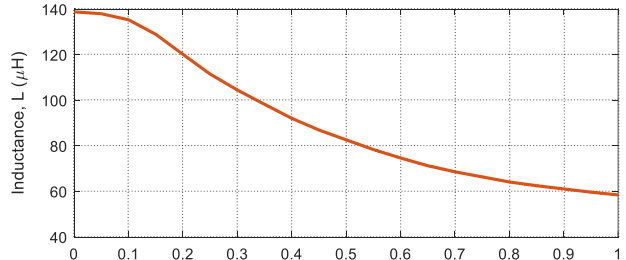

(a)

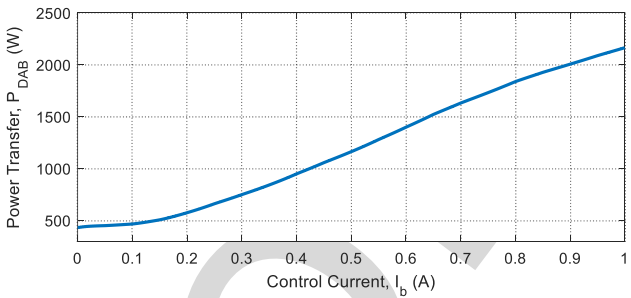

(b)

Fig. 12. Effect of inductance variation on power transfer in the proposed DAB converter. (a) Inductance as a function of bias current. (b) RMS power transfer in DAB converter as a function of bias current.

of a dc component in the current will imply lower dc power losses, while, on the other hand, a higher frequency current waveform would contribute to increase the eddy current losses due to skin and proximity effects. And second, the ac current implies that an induced voltage could be reflected from the ac main winding and generated across the dc control windings which would drastically affect the design of those windings to be able to handle higher levels of induced voltage. This, therefore, constrains the arrangement of the VI windings such that the two dc control windings be serially connected in opposite polarity to cancel the induced voltages due to the ac main winding.

In conclusion, once the significant elements of the electromagnetic circuit are defined, the developed model can thus be used to study the full dc-dc power converter system.

\section{Proposed Power Flow Scheme}

This section is intended to demonstrate the proposed structure of power flow in the DAB converter by using the variable magnetic element as well as PS modulation. Special emphasis is given to the improvements brought about by replacing the fixed inductor in a conventional DAB converter by a variable one.

\section{A. Control of Power Transfer Using VI}

As mentioned in the previous section, the value of the effective inductance in the converter can be varied by means of biasing the operation point on the $B(H)$ curve of the magnetic core, using a $\mathrm{dc}$ bias control current flowing through the control windings. For reference, Fig. 12(a) depicts the simulated inductance variation as a function of the bias control current, when varying the current from 0 to $1 \mathrm{~A}$ in steps of $0.1 \mathrm{~A}$. The inductance value is observed to decrease from a maximum value of $140 \mu \mathrm{H}$ at no saturation, to $60 \mu \mathrm{H}$ at full saturation, which is around $60 \%$ variation range. Fig. 12(b), in turn, shows the resulting variation in the power transfer in the DAB converter as a function of the dc bias control current, the power level varies from the nominal value of 500 to $2100 \mathrm{~W}$, which is nearly three times greater than the nominal. 


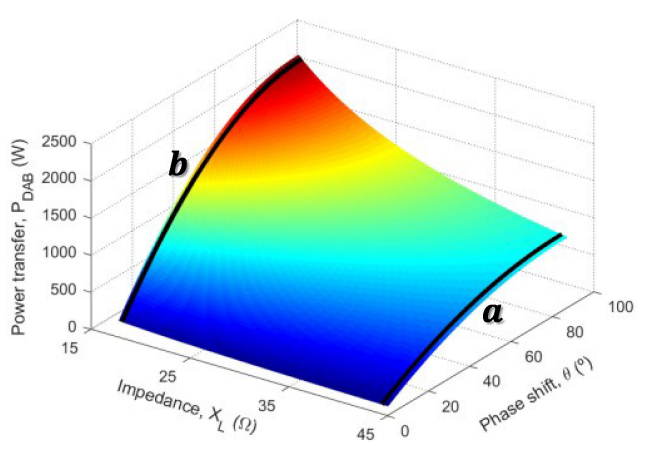

Fig. 13. Power transfer in a DAB converter as a function of inductance and PS.

This illustrates that the VI can serve as a control parameter for the power transfer in the converter.

Fig. 13 further adds a third dimension to depict the effect of PS as a control parameter, in combination with the inductance variation. As it can be observed, at a certain inductance value, as the PS increases, the power transfer in the DAB converter increases correspondingly, based on (1). However, this increase of the power becomes less significant as the inductance value increases.

This can be clarified by comparing the operation at the two different curves $\boldsymbol{a}$ and $\boldsymbol{b}$. At $0 \mathrm{~A}$ bias current, where $L$ is maximum, as the PS increases, the power transfer follows the curve $\boldsymbol{a}$. A relatively small variation in the power is possible. However, at $1 \mathrm{~A}$ bias, where $L$ is minimum, as the PS increases, the power transfer follows the curve $\boldsymbol{b}$, which shows more than double the variation of the power transfer compared to that at curve $\boldsymbol{a}$.

From another side, the figure also defines the limitations imposed by the PS control parameter on the variable inductance one. The figure clarifies that for higher PS, the decrease of the inductance can achieve larger output power compared to lower PS. In other words, the effect of the bias control current of the VI is increased as the PS between bridges increases toward the maximum. Therefore, selecting the inductor value for a specific operation range is a critical process in a $\mathrm{DAB}$ converter design.

\section{B. Linearization of the Control Function}

As explained in the previous section, the power transfer of a DAB converter is regulated by (1), as a function of several parameters. Fig. 14(a) shows the power transfer characteristics in the DAB converter as a function of the PS. It can be observed that the power transfer increase is approximately linear with the increase of PS until a certain limit, after which the rate of increase of power drops following a sinusoidal waveform until reaching the maximum power transfer at $90^{\circ} \mathrm{PS}$ angle. This characteristic operation is intrinsic to the PS DAB converter, and thus, to control the power transfer in the converter, the PS is used as the primary control parameter. By manipulating (1), the following expression is obtained:

$$
\theta=\frac{\pi}{2}\left(1 \pm \sqrt{1-\frac{4 P_{\mathrm{DAB}} X_{L} r_{t}}{\pi V_{\mathrm{in}} V_{\mathrm{out}}}}\right)
$$
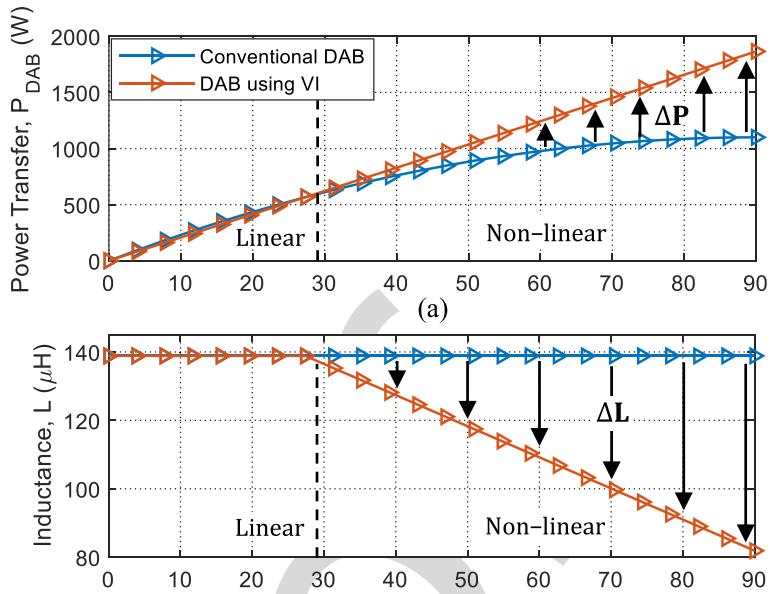

(b)

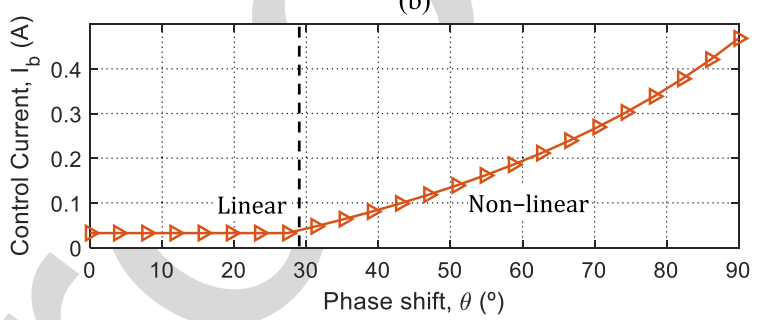

(c)

Fig. 14. Linearization of power transfer characteristics in the proposed DAB converter using VI. (a) Power transfer, (b) inductance, and (c) bias control current command as a function of PS angle.

which explicitly defines the PS angle $\theta$ as a function of the output power $P_{\mathrm{DAB}}$. As it can be observed, (5) presents a nonlinear equation, introducing difficulty in implementation and excessive processing time, as well as affecting the dynamic performance of the digital controller. Therefore, it is promising to use the inductance as an extra control parameter to allow the linearization of the power transfer function, and thus achieving a linear control function, the merits of which will be discussed as follows.

Fig. 14(a) illustrates the nonlinear power transfer curve of the conventional DAB converter, compared to the linearized one achieved by making use of the variation in the inductance. As it can be observed, the linear power transfer curve coincides, approximately, with the nonlinear curve in the range of $\theta=$ $0^{\circ}-30^{\circ}$, which is illustrated on the plots as the linear region.

Therefore, the phase-to-power relationship of the linear power curve can be defined by the slope of the straight line. Consequently, by substituting the values of the power at $0^{\circ}$ and $30^{\circ}$ in (1), the linear power-to-phase relationship can be defined by

$$
\theta_{\text {lin }}=\frac{X_{L} \cdot I_{\mathrm{out}} \cdot r_{t}}{V_{\mathrm{in}}(5 / 6)}
$$

The inductance is constant during the linear region, as demonstrated in Fig. 14(b), while in order to increase the power transfer by $\Delta P$ and keep the linearity of the function, the inductance is reduced by $\Delta L$, as illustrated in the figure. The inductance is computed as a function of the power transfer and PS angle, as 


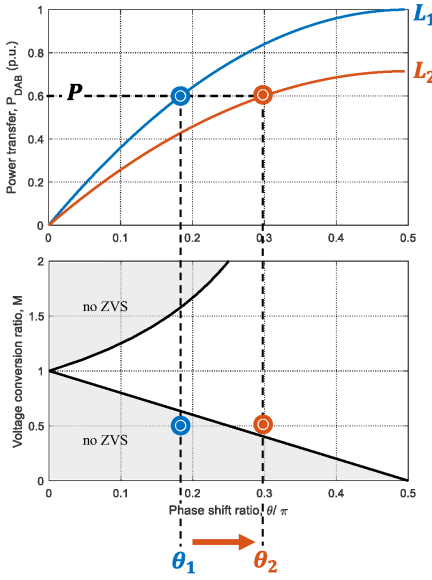

(a)

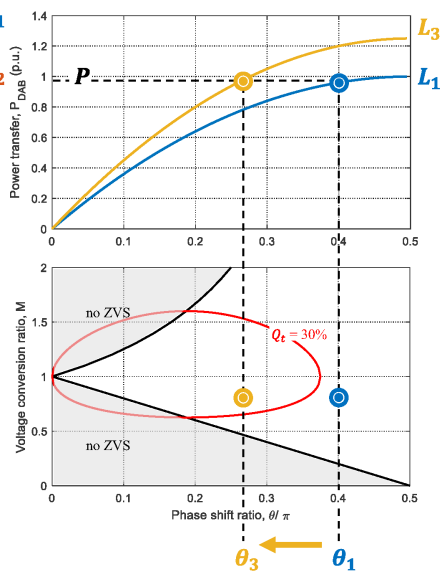

(b)
Fig. 15. Power transfer in the DAB converter (top plot), and the corresponding voltage conversion ratio (bottom plot), showing two operation conditions: (a) light load and (b) heavy load.

shown in the following, which is derived from (1):

$$
L=\frac{V_{\mathrm{in}} V_{\mathrm{out}}}{2 \pi f \cdot r_{t} \cdot P_{\mathrm{DAB}}} \theta\left(1-\frac{\theta}{\pi}\right) .
$$

In order to achieve the calculated inductance variation in the magnetic device, the bias current is increased, as shown in Fig. 14(c).

\section{Combination of Inductance Variation With Modulation Schemes to Improve the Converter Efficiency}

At light loading condition, i.e., $V_{\text {out }} \ll r_{t} V_{\text {in }}$, two issues take place which contribute significantly to increase the power losses and thus to reduce the efficiency in this operation region. First, the loss of ZVS due to insufficient energy required to charge and discharge the MOSFET capacitors causing elevated switching losses [1]. And second, relatively small PS values interfere with the deadtime range causing a phenomenon called "voltage polarity reversal" [5], [36] and limiting the minimum power transfer. Those two issues cause an increase in the reactive power component and consequently, reduce the efficiency at this load range. In addition, at high loading condition, i.e., $V_{\text {out }} \gg r_{t} V_{\text {in }}$, relatively large circulating currents take place, causing an increase in conduction losses and elevating reactive power losses [4], [22].

In this context, varying the inductance to have a relatively higher value at light load allows for increasing the PS and maintaining ZVS for a wider range, as well as avoiding the interference with the deadtime. And, on the other hand, decreasing the inductance value at heavy load allows for reducing the circulating currents.

To demonstrate the idea, Fig. 15 explains the two conditions of low and high loading. The figure shows the power transfer in the DAB represented in per unit (p.u.) values, and the corresponding voltage conversion ratio. The different boundaries of ZVS and circulating currents are indicated to clarify the sought operation zone. Fig. 15(a) shows that at a low loading, a small PS angle $\theta_{1}$ could lead the converter to operate in a no ZVS region. However,

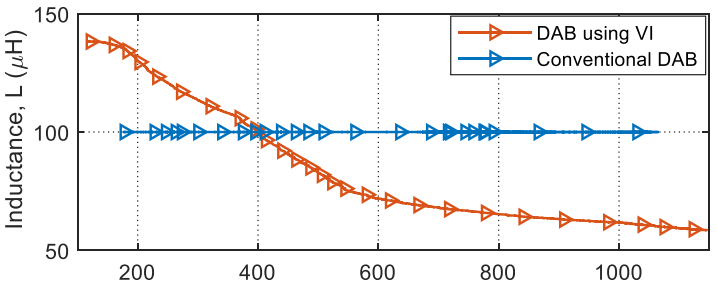

(a)

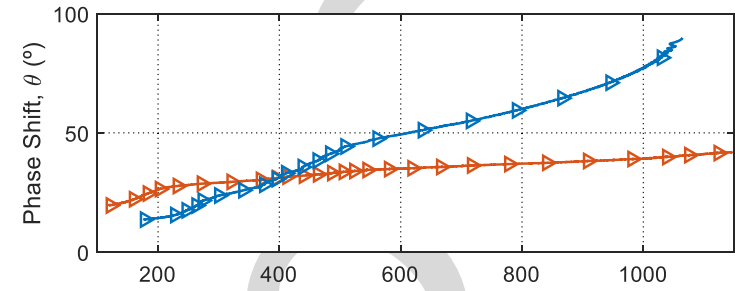

(b)

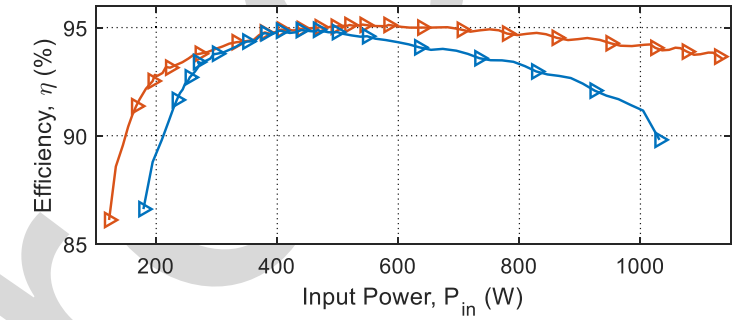

(c)

Fig. 16. Efficiency of DAB converter using VI. (a) Inductance value, (b) PS, and (c) converter efficiency as a function of output power transfer.

to achieve the same power level $P$, the inductance value can be increased from $L_{1}$ to $L_{2}$. This allows increasing the PS angle to $\theta_{2}$ to be able to operate under ZVS once again. In the same manner, Fig. 15(b) shows that at a high loading, a large PS angle $\theta_{1}$ could lead the converter to operate under high circulating currents, exceeding the required percentage reactive currents boundary. However, to achieve the same power level $P$, the inductance value can be decreased from $L_{1}$ to $L_{3}$. This allows decreasing the PS angle to $\theta_{3}$ to be able to operate within the reactive boundaries once again.

Using the simulation model, this analysis is verified by comparing the efficiency of the full DAB converter using the conventional constant inductance against that using the variable inductance. Fig. 16 illustrates the simulation results. Fig. 16(a) compares the inductance in a conventional DAB converter to that of the proposed DAB using VI. While the conventional inductance is observed to be constant at $100 \mu \mathrm{H}$, the VI changes its value from the maximum of $144 \mu \mathrm{H}$ to the minimum of around $50 \mu \mathrm{H}$. The PS angle is calculated in the simulation model by substituting $L$ in (5), as illustrated in Fig. 16(b). It can be observed that, in the case of the constant inductance, the PS varies between the minimum and maximum values to adjust the power transfer. The small PS angles applied at light loading implicate a loss in the ZVS and interference with the deadtime. While at a high loading condition, the large PS angles elevate the reactive power losses. On the other hand, observing the PS curve in the case of using variable inductance, it can be observed that the variation is limited to a small range, $20^{\circ}<\theta<50^{\circ}$. This 


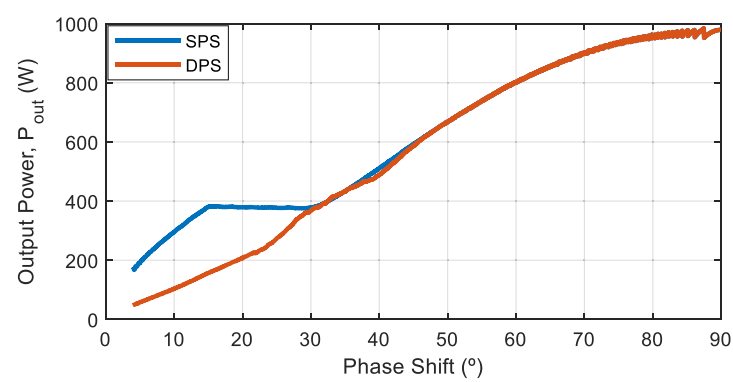

Fig. 17. Output power delivered by the DAB converter as a function of the PS angle, comparing SPS to DPS modulation strategies.

enhances the efficiency under the latter conditions of operation, as observed in Fig. 16(c).

Another feature is that the variation of the inductance can allow for achieving ZVS over the full operation range if combined with a modulation scheme such as the dual-PS (DPS) [37], [4]. This can be observed in Fig. 17, which compares the power flow in the DAB converter for the cases of SPS and DPS modulations.

More complicated modulation schemes are studied in some literature works [3], [6], [7], which aim at achieving ZVS over the full operation range of the converter. An example of those strategies is the triple-PS (TPS) [8]. Although the TPS is reported to provide the widest ZVS operation [8], it is also reported to pose more variables to the control function. This implies a more complicated implementation of the control calculations and requires higher processor capabilities [9]. In this manner, the use of VI can provide a good compromise between complexity and, on the other hand, the ease and time of implementation of the control function.

Therefore, based on the previous analysis, the proposed model takes advantage of combining the DPS modulation scheme with the variation of inductance in order to extend the range of the ZVS to the full operation of the converter.

\section{Power Flow Schemes Based on the Operation Mode}

It is necessary when using several control parameters to decide the combination of those parameters in the system control function. In the case of the DAB converter, the objective is to control the power transferred between the input and output ports. Specific to this converter, the characteristics of the power flow impose some restrictions on the operation over wide load ranges, as it has been discussed in the previous sections. Therefore, two modes of operation can be applied to combine the inductance with the PS as control parameters.

In the first mode, Mode I, the inductance value is used as the primary control parameter. This is a simplified mode where the power transfer can be adjusted throughout the full operation range by means of the variation of the inductance between the maximum and the minimum values. This mode assumes that the load, which the converter will supply, has a known (or predictable) profile as a function of time, which is the case in some applications, for example, in steel rolling mills. In such a condition, also the bias control current, which is responsible for adjusting the inductance value, can be defined as a fixed profile

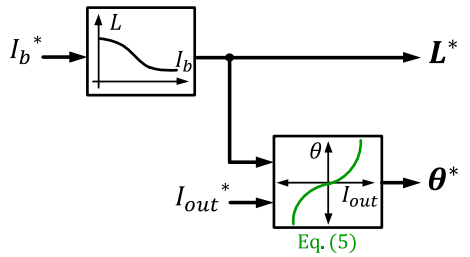

Fig. 18. Combination of variable inductance with PS to control the power flow in the DAB converter: Mode I.
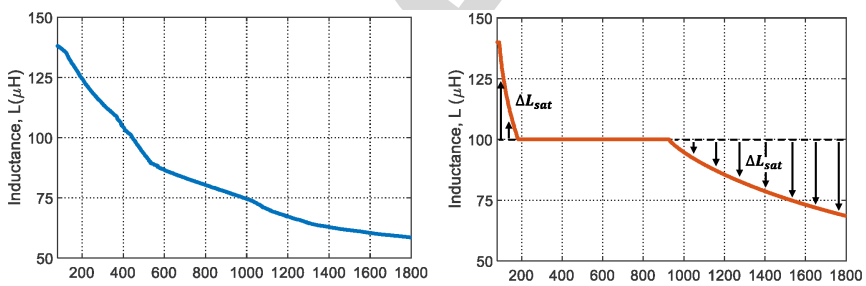

Output Power, $\mathrm{P}_{\text {out }}(\mathrm{W})$

(a)

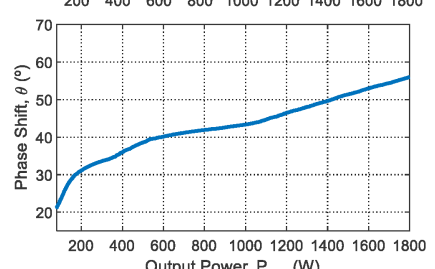

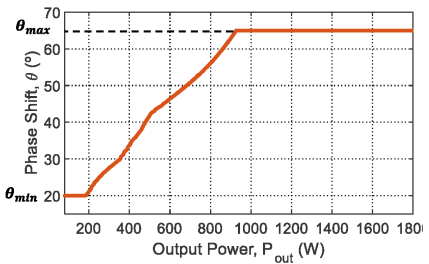

(b)
Fig. 19. Inductance (top) and PS angle (bottom) as a function of the output power in the DAB converter using VI. (a) Mode I. (b) Mode II.

as a function of time. This can allow disregarding the dc-dc converter required to control the bias current of the inductor and instead use a programmable source. Such simplification can be advantageous in some applications to reduce the number of sensors and unnecessary digital control computations. However, since the operation is based merely on open-loop calculations, some differences might occur between the required power and the provided one. This difference can be provided by the secondary control parameter, which is the PS angle between the converter bridges. To achieve a specific power, the required PS is, therefore, computed based on the current-to-phase relationship derived from (5) by replacing $P_{\mathrm{DAB}}$ by $V_{\text {out }} I_{\text {out }}$. Therefore, this mode of operation can be represented by the simplified block diagram in Fig. 18. The resulting waveforms are illustrated in Fig. 19(a). In this case, the load profile simulated is a linear ramp increasing as a function of time, which implies that the bias control current is a ramp proportional to the load profile. As $I_{b}$ is increased linearly, the inductance value is observed to reduce from maximum to minimum values, as it can be seen in the top plot. The variation of the PS angle, shown on the bottom plot, is observed to be bound in a small range, since it compensates the power difference between the designed and the required inductance values at each operation point.

If the inductor is well designed for the application, then, besides the simplicity of implementation, this mode will achieve two advantages. First, the phase-to-current relationship is always in the linear region, as it was mentioned in the previous section. And second, the value of the PS angle, compared to the conventional DAB, is higher at light loads to keep ZVS, and lower at 


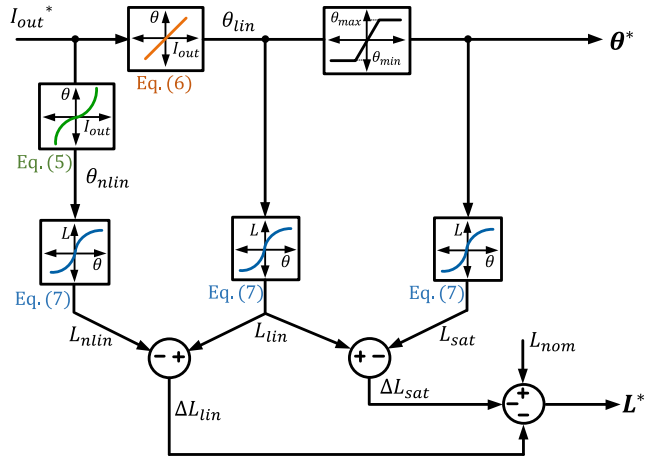

Fig. 20. Combination of variable inductance with PS to control the power flow in the DAB converter: Mode II.

heavy loads to reduce circulating currents. A better efficiency can thus be achieved over a wider load range.

On the other hand, in the second mode, Mode II, the PS angle is considered as the primary control parameter. This is more generic where the power transfer in the DAB converter is adjusted by means of the PS angle, while the inductance is used to achieve some enhancements to the power flow characteristics. This mode assumes that the load can vary in wide and unpredictable profiles, for example, in grid-tied applications, such as the SST-based microgrid paradigm under study, where the system exhibits disturbances of the demanded power and is susceptible to fault conditions. In such a condition, the reference PS angle $\theta_{l \text { in }}$ is calculated as a function of the demanded output current, $I^{*}$ out. This is done by using the linear current-to-phase relationship, previously expressed in (6), to keep the simplicity in digital implementation. The nonlinearity is thus compensated by the inductance variation. For this, the required variation of the inductance $\Delta L_{\text {lin }}$ value is computed by using the phase-toinductance relationship in (7). Meanwhile, the reference of the PS angle is always saturated to a minimum and a maximum value $\theta_{\min }$ and $\theta_{\max }$, respectively, whether to the limits of ZVS and reactive currents or during transient events.

The minimum PS limit is defined by the deadtime and the minimum phase angle that achieves ZVS [35]. In this case, since the proposed model applies DPS to achieve ZVS at light load, then the minimum PS is simply the angle corresponding to the deadtime $1 \mu \mathrm{s}$, which corresponds to $18^{\circ}$ at $50 \mathrm{kHz}$ switching frequency. And, the maximum PS is $65^{\circ}$. Previous works [21] in the literature have limited the PS angle to $65^{\circ}$, due to the nonlinearity of the phase-power function, as it can be recalled from Fig. 14(a), where it can be observed that after $65^{\circ}$, the rate of increase of power drops. The amount of saturation in the PS angle is, hence, compensated by the inductance variation $\Delta L_{\text {sat }}$, which is computed by using (7). Fig. 20 shows a block diagram of the operation in Mode II. Also, the resulting waveforms are illustrated in Fig. 19(b), which when compared to Fig. 19(a), it can be observed that the PS is, similarly, limited by $\theta_{\min }$ and $\theta_{\max }$, and has a linear relationship as a function of current. Compared to Mode I, this mode involves more computations. However, it provides the two formerly mentioned advantages keeping a linear relation between the PS and the current as well

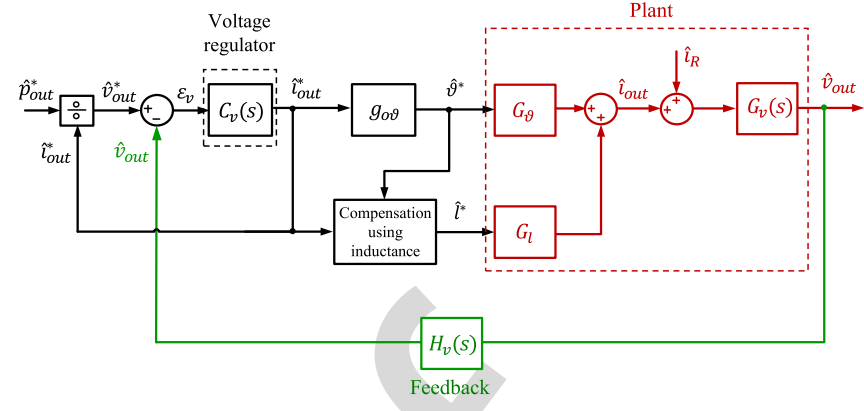

Fig. 21. Closed-loop control of DAB converter using inductance and PS as two control parameters.
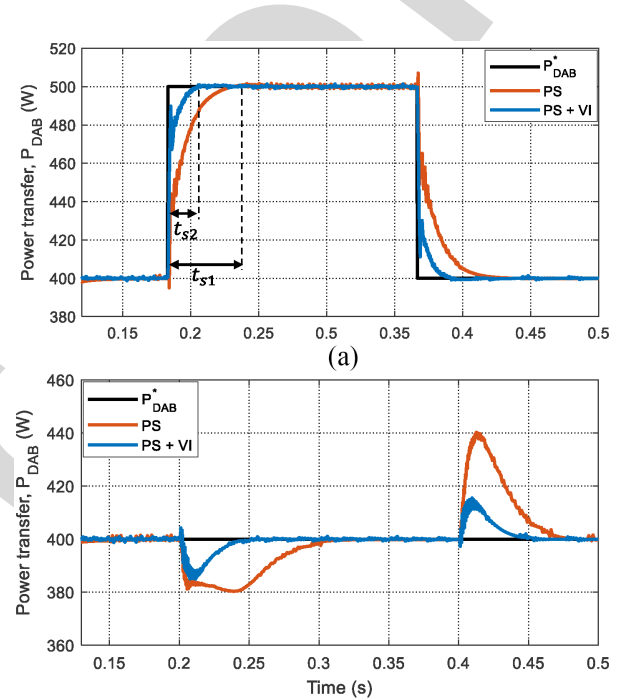

(b)

Fig. 22. Dynamics of PI controller comparing the control using the PS to that combining PS and VI.

as enhancing the efficiency over a wide load range. And, furthermore, making use of this combination of control parameters to design a closed-loop control for the system could allow to address unexpected system transients or disturbance events in an improved manner, as it will be demonstrated in the following section.

\section{E. Closed-Loop Control}

The closed-loop structure is based on the combination of the variable inductance and the PS as two control parameters. The structure is based on Mode II being the generalized operation mode, as shown in Fig. 21. The phase-current and inductancephase relationships are implemented based on (1). The dynamic performance of the controller is tested by introducing a step in the reference power. The step response of the control combining PS and VI is compared against that using PS only. Fig. 22 shows that the time response is smaller in case of adding the VI as a control parameter. This is due to the use of VI to compensate the saturation and linearization of the phase-to-current relationship as explained in the previous sections. Referring to the previous analysis, emphasis can be given to the advantages of using these modes of operation compared to the conventional DAB 


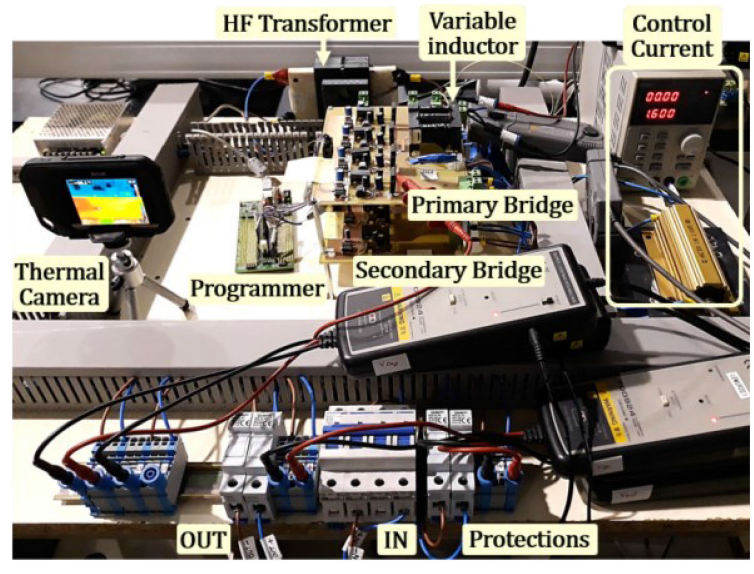

Fig. 23. Experimental setup.

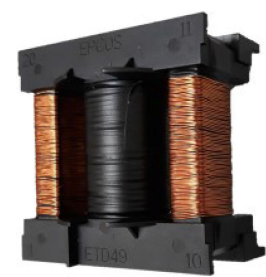

Fig. 24. Variable inductor prototype.

converter. First, the PI controller is tuned based on the linear phase-to-current relationship, which simplifies the mathematical operations and thus, decreases the processing time of the implemented control function. Second, the PS limitation does not affect the minimum power that can be transferred by the converter, since the inductance value is increased to compensate this. Third, circulating current can be significantly reduced due to the reduction of the PS angle especially at higher power, which is compensated by the decrease of the inductance value. And finally, the ZVS at light loads could be maintained by allowing the storage of enough energy in the inductor to charge and discharge the switches' capacitors.

\section{EXPERIMENTAL RESULTS}

\section{A. Experimental Platform}

A test platform has been developed to validate the electromagnetic model and the system analysis. The platform consists of a power stage and a programmer stage. The power stage includes the DAB converter as well as the VI. The DAB is composed of two full-bridge converters implemented using SiC MOSFETs. Each bridge is implemented on a separate PCB. The PCBs for the primary and secondary bridges are stacked on top of each other, with the VI prototype mounted on the primary bridge PCB, as seen in Fig. 23. The specific details of the design and operation parameters are summarized in Table II. Also, Fig. 24 shows the developed VI prototype. To supply the control winding of the VI, a variable dc voltage source is used in series with a resistor to have a maximum of $1.5 \mathrm{~A}$ dc bias control current. Additionally, a film capacitor is connected in parallel with the source terminals
TABLE II

SPECIFICATIONS OF THE TEST SETUP

\begin{tabular}{|c|c|c|}
\hline \multicolumn{3}{|c|}{ Power Stage } \\
\hline \multirow{6}{*}{$\begin{array}{c}\text { DAB } \\
\text { Converter }\end{array}$} & Rated power & $2 \mathrm{~kW}$ \\
\hline & Input voltage & $200 \mathrm{~V}$ \\
\hline & Output voltage & $250 \mathrm{~V}$ \\
\hline & Peak inductor current & $15 \mathrm{~A}$ \\
\hline & Switching frequency & $50 \mathrm{kHz}$ \\
\hline & Deadtime & $1 \mu \mathrm{s}$ \\
\hline \multirow{6}{*}{$\begin{array}{l}\text { Variable } \\
\text { Inductor }\end{array}$} & Core shape and size & ETD49/25/16 \\
\hline & Magnetic material & N87 \\
\hline & Inductance variation range & $144 \mu \mathrm{H}-50 \mu \mathrm{H}$ \\
\hline & DC voltage source & $0-30 \mathrm{~V}$ \\
\hline & Current limiting resistor & $10 \Omega$ \\
\hline & Peak DC control current & $3 \mathrm{~A}$ \\
\hline \multicolumn{3}{|c|}{ Programmer Stage } \\
\hline Hardware & $\mathrm{C2000}^{\mathrm{TM}}$ Real-time MCU & TMS320F28335 [29] \\
\hline Software & $\begin{array}{c}\text { Integrated development } \\
\text { environment }\end{array}$ & Code Composer Studio [30] \\
\hline
\end{tabular}

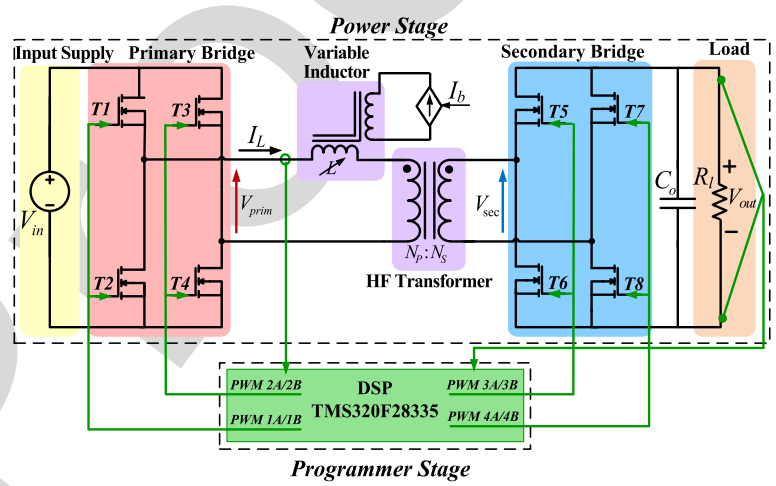

Fig. 25. Schematic of experimental setup.

to ensure that the voltage, and consequently the dc current, will be as smooth as possible.

The HF isolation is achieved by an HF planar transformer. The transformer ratings as well as the measured model parameters have been already detailed in Section III. The tests carried out consider the DAB converter feeding a resistive load. The load is connected to the output dc-link of the DAB converter.

On the other hand, the digital control stage consists of a TMS320F28335 controlCARD from Texas Instruments, which is a C2000 32-bit microcontroller unit (MCU) [29]. The controlCARD is mounted on a basis peripheral board for hardware development. The software platform used is the Code Composer Studio (CCS) from Texas Instruments [30]. The programmed code is configured to realize two main tasks-providing the switch modulation and processing the feedback measurements.

The hardware connections of the two stages, in addition to the firing pulses, are illustrated in Fig. 25.

\section{B. Impact of Inductance on Power Transfer in DAB Converter}

The inductance value is varied by means of adjusting the dc bias control current $I_{b}$. As the control current increases, the magnetic core will approach saturation. The permeability of the magnetic material will thus decrease, causing an increase in the reluctance and eventually, a decrease in the value of 


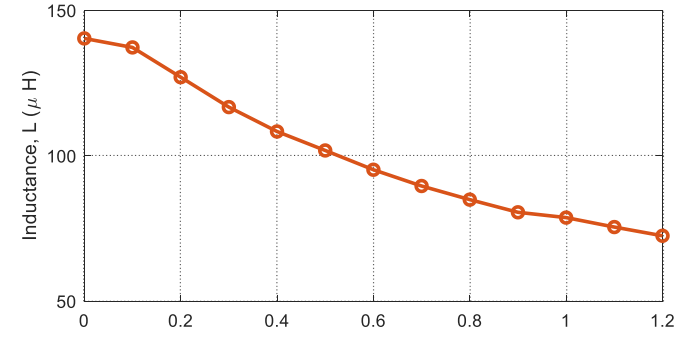

(a)

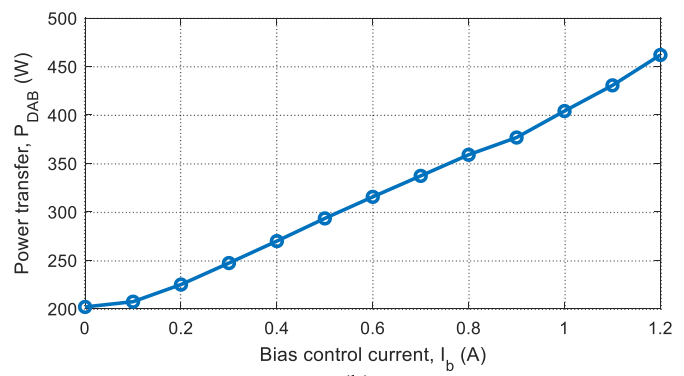

(b)

Fig. 26. Experimental results. Effect of inductance variation on power transfer in the DAB converter under buck operation mode. (a) Measured inductance and (b) power flow as a function of the bias control current.

the inductance measured from the main winding terminals. The decrease in the value of the inductance, in its turn, results in a decrease of the inductor impedance under a constant switching frequency of the converter, governed by (1). Specific to the DAB converter, the operation mode can vary depending on the levels of the input and the output voltage. The voltage conversion ratio $M$ has been previously defined to differentiate those modes of operation, such that $M=1$ refers to $V_{\text {in }}=V_{\text {out }} / r_{t}$, while $M<1$ refers to $V_{\text {in }}>V_{\text {out }} / r_{t}$ which is identified as the buck mode, and $M>1$ refers to $V_{\text {in }}<V_{\text {out }} / r_{t}$ which is identified as the boost mode.

Two experiments are thus carried out to identify the effect of the inductance variation on the power transfer in both latter conditions of operation. Based on this configuration, the power is transferred from the primary bridge to the secondary one, i.e., to the load, while the PS is kept constant at the maximum value of $\theta=90^{\circ}$. The dc bias current supplying the VI control windings is increased from 0 to $1.2 \mathrm{~A}$ in steps of $0.1 \mathrm{~A}$. The power transfer can, hence, be computed using the measurements of the voltage across the VI main winding, and the current flowing through it. The inductance value is, similarly, computed from the measured voltage and current, by using the rms of the first harmonic component of the waveforms [26].

Fig. 26 illustrates the measured inductance and the power transfer in the DAB as a function of the dc control current for the buck mode, along with the corresponding instantaneous voltage and current waveforms measured across the inductor main winding illustrated in Fig. 27. Meanwhile, the same experiment was repeated also for a higher loading condition, operating under the boost mode to reach a maximum power level of $1.8 \mathrm{~kW}$, as shown in Fig. 28. The peak inductor current is boosted from $5 \mathrm{~A}$ at no saturation to $15 \mathrm{~A}$ at full saturation of the magnetic core, as it can be observed in Fig. 29.

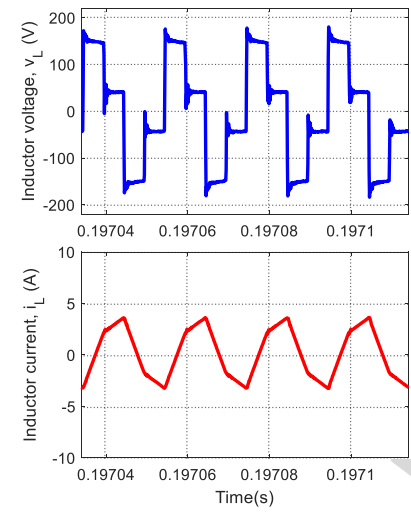

(a)

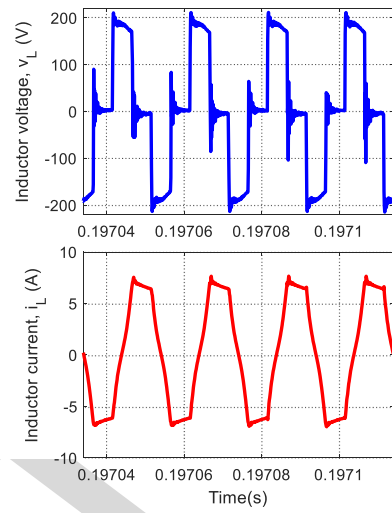

(b)
Fig. 27. Open-loop experimental results for DAB operating in buck mode the ac inductor voltage (top) and current (bottom) for three levels of dc bias control current: (a) $0 \mathrm{~A}$ and (b) $1.2 \mathrm{~A}$.

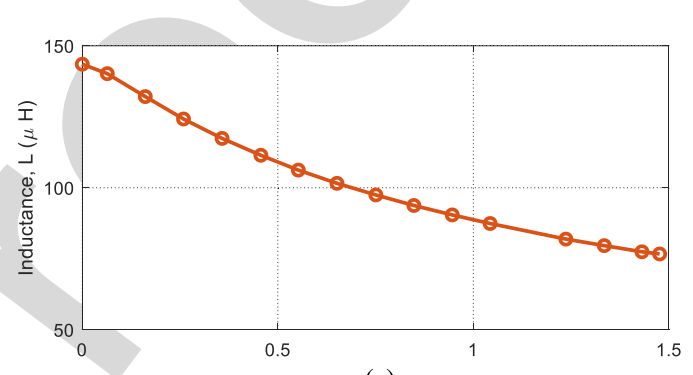

(a)

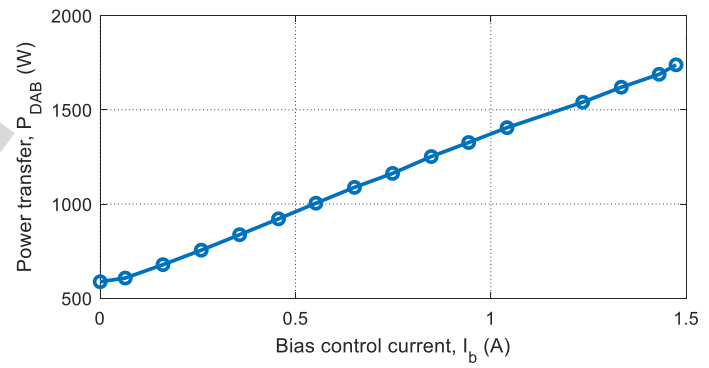

(b)

Fig. 28. Experimental results. Effect of inductance variation on power transfer in the DAB converter under boost operation mode. (a) Measured inductance and (b) power flow as a function of the bias control current.

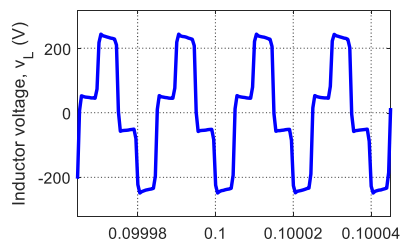

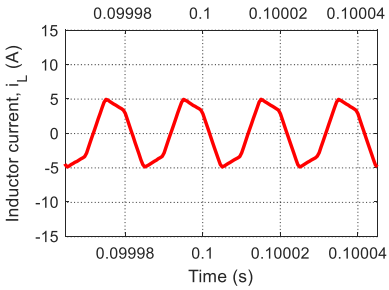

(a)
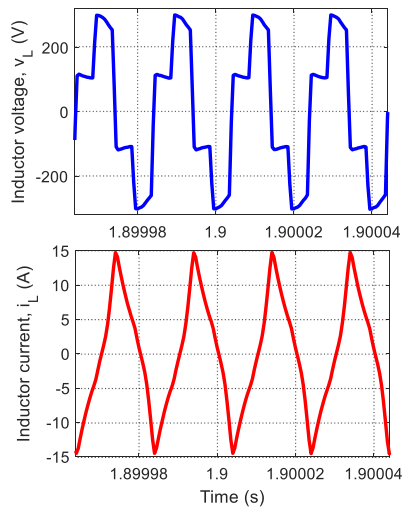

(b)
Fig. 29. Open-loop experimental results for DAB operating in boost mode The ac inductor voltage (top) and current (bottom) for three levels of dc bias control current: (a) $0 \mathrm{~A}$ and (b) $1.5 \mathrm{~A}$. 


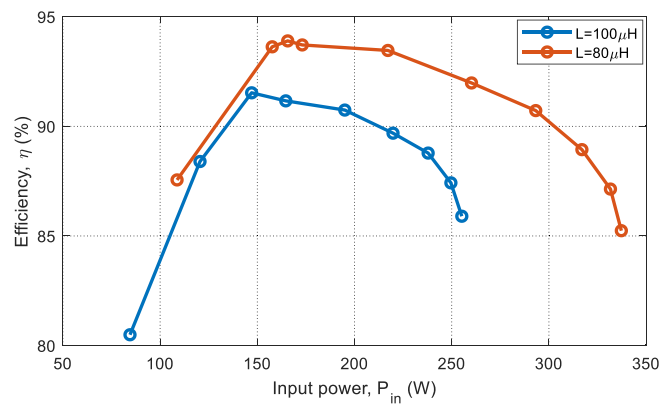

Fig. 30. Experimental results. Efficiency curves when using PS to control the power transfer in the DAB converter prototype.

\section{Efficiency Curves}

After analyzing the operation of the DAB converter prototype using the VI, the merits of using the variable inductance for improving the operation of the DAB converter are explored in this section. To achieve this, several measurements have been carried out for recording the efficiency curves of the full converter as a function of the power transferred. To conceive a comprehensive comparison, three operation scenarios are studied, as explained below.

1) PS Angle as the Primary Control Parameter: The first is the conventional scenario of controlling the power transfer in the DAB by means of varying the PS between the primary and the secondary bridges, while keeping the inductance value constant. The PS angle between the primary and secondary bridges is varied from $0^{\circ}$ to $90^{\circ}$ to transfer power from the input to the load, while the inductance value is kept constant at $100 \mu \mathrm{H}$.

For the experiments carried out herein, the DAB converter is operated in the buck mode, whereby the test conditions presented in the previous section are replicated. Fig. 30 illustrates the efficiency curves calculated for both experiments as a function of the input power. It can be observed that, for $80 \mu \mathrm{H}$ inductance, the efficiency value ranges from $85 \%$ to $94 \%$, while the peak value is shifted to $165 \mathrm{~W}$. Comparing both efficiency curves, it can be observed that using a lower inductance achieves a higher efficiency, especially as the level of power increases. This occurs at high loading condition, since reducing the inductance value allows transferring the same power with a smaller PS angle, which implies a smaller angle between the voltage and current applied to the transformer, and consequently, lower reactive power losses.

2) Inductance as the Primary Control Parameter: On the other hand, the second scenario studied is to control the power transfer in the DAB by means of variation of the inductance value through controlled saturation, as discussed previously, while in this case, the PS between the bridges is kept constant. Similar to the previous analysis, this experiment is repeated for two different values of the PS angle, $50^{\circ}$ and $90^{\circ}$. Fig. 31 illustrates the efficiency curves as a function of the input power for both values of PS angle. In this case, the efficiency curves are observed to be more flat, presenting smaller efficiency fluctuation between the maximum and minimum values experienced. As the PS angle is reduced from $90^{\circ}$ to $50^{\circ}$, the efficiency values are increased by

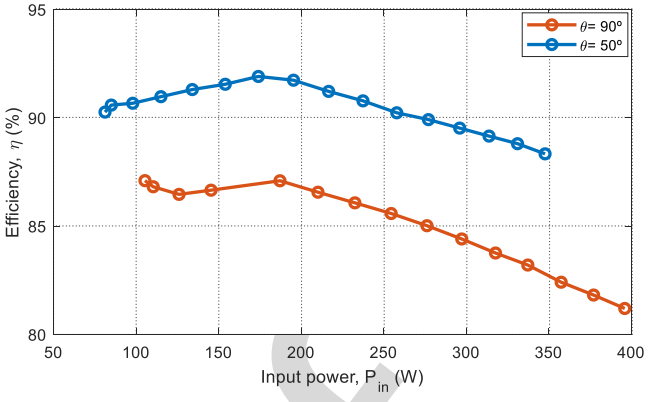

Fig. 31. Experimental results. Efficiency curves when using inductance to control the power transfer in the DAB converter prototype.

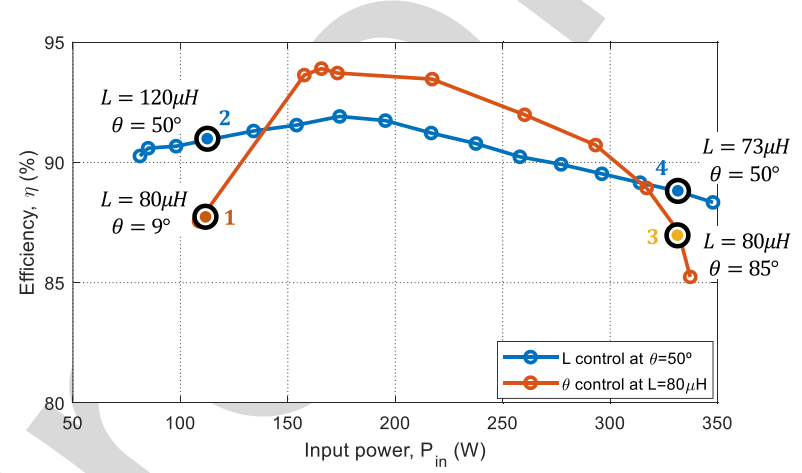

Fig. 32. Experimental results. Efficiency curves when combining inductance with PS to control the power transfer in the DAB converter prototype.

almost 6\%, in a nearly linear translation of the efficiency curve upward. This conclusion is, similarly, attributed to the reduction of the circulating currents and consequently, the reactive power losses, which eventually leads to higher efficiency.

3) Combining PS and Inductance as Control Parameters: Finally, both the previous scenarios are combined to compare the efficiency curves of the full DAB converter, with the aim of demonstrating the efficiency improvement accomplished by combining both parameters to control the power transfer. Fig. 32 illustrates two efficiency curves; one is measured at the operation scenario whereby the PS angle is used as the control parameter while keeping the inductance constant at $80 \mu \mathrm{H}$. The other curve is measured at the operation scenario whereby the inductance is used as the control parameter while keeping the PS constant at $50^{\circ}$. Those are the curves which presented the highest efficiency during the previous efficiency comparative tests.

Within the conditions of operation of the experiment carried out, at the specific input voltage of $100 \mathrm{~V}$ and load value of $50 \Omega$, the nominal value of the power transfer is approximately $200 \mathrm{~W}$, which can be calculated by (1). Around this value, it is apparent that using the PS as the control parameter achieves higher efficiencies. On the other hand, for low and high loading conditions, the use of the VI achieves higher efficiency values. To clarify this observation, two points are selected for comparison under each of the operation conditions such that the input power level is kept constant, and thereby, a constant voltage conversion ratio $M$. Hence, points $\mathbf{1}$ and $\mathbf{2}$ are compared for the low loading operation condition, while points $\mathbf{3}$ and $\mathbf{4}$ are compared for the high loading condition. 


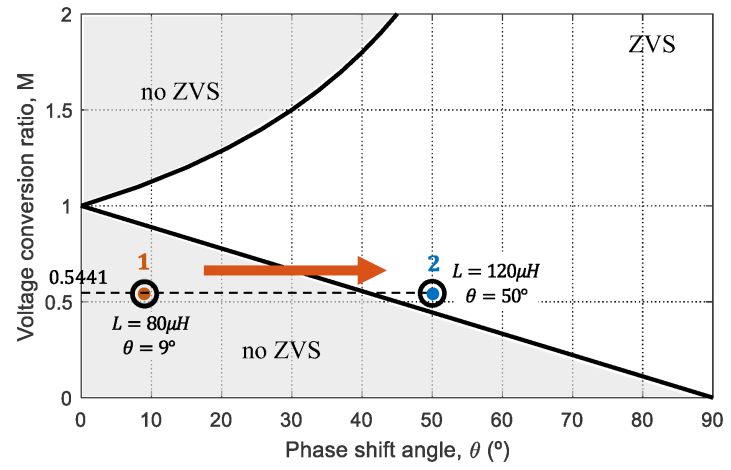

Fig. 33. Experimental results. The voltage conversion ratio $M$ as a function of the PS angle $\theta$ in degrees, illustrating the boundaries of ZVS in the DAB converter, for comparing the experimental operation points $\mathbf{1}$ and $\mathbf{2}$.
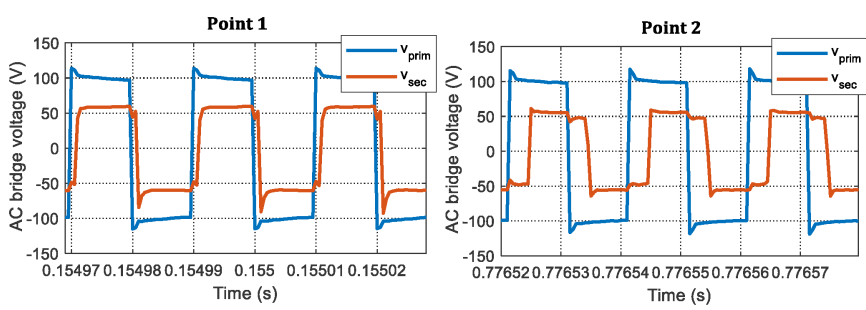

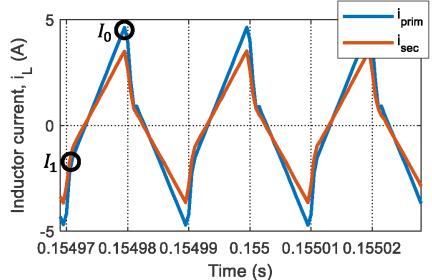

(a)

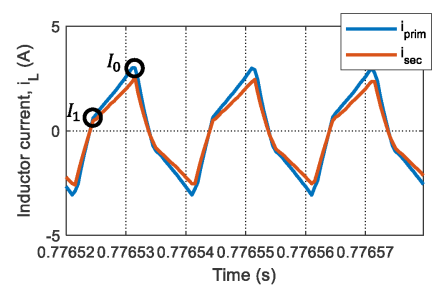

(b)

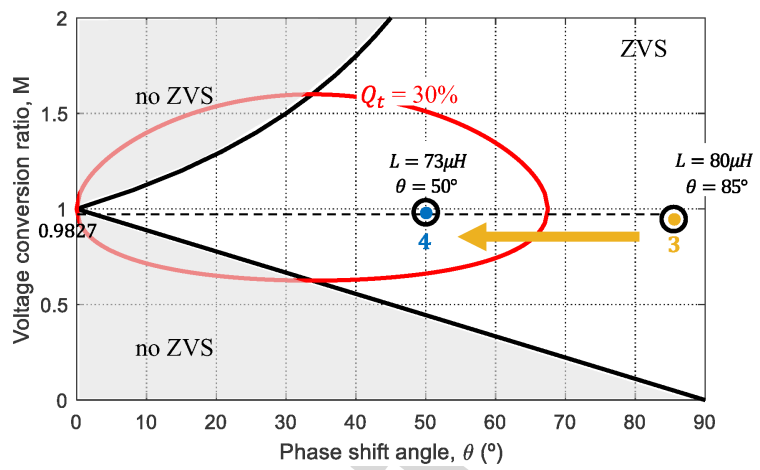

Fig. 35. Experimental results. The voltage conversion ratio $M$ as a function of the PS angle $\theta$ in degrees, illustrating the boundaries of ZVS in the DAB converter, for comparing the experimental operation points $\mathbf{3}$ and $\mathbf{4}$.
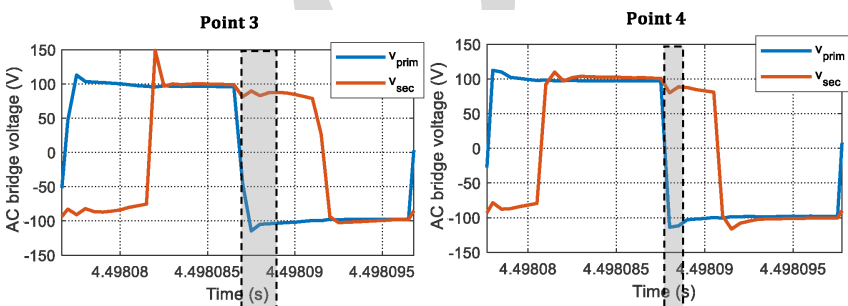

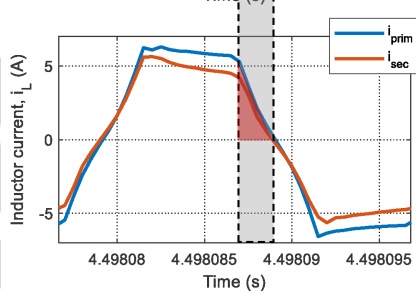

(a)

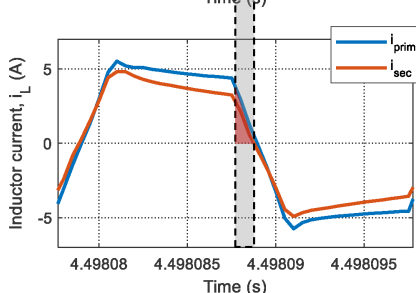

(b)

Fig. 34. Experimental results. The measured ac primary and secondary bridge voltages (top) and the corresponding inductor current measured at the primary and secondary (bottom), for comparing the experimental operation at: (a) point $\mathbf{1}$ and (b) point 2.

To compare the points $\mathbf{1}$ and $\mathbf{2}$, Fig. 33 is generated for $M$ as a function of $\theta$, in degrees, while illustrating the ZVS boundaries. This analysis is an experimental application of the theoretical study performed previously in Section II, precisely recalling Fig. 15(a). It can be observed that point 1 presents an operation condition of the converter at no ZVS.

This explains the low efficiency obtained when comparing to point $\mathbf{2}$. Therefore, moving to point $\mathbf{2}$ can allow to bring the converter operation again into the ZVS region. This is due to the fact that increasing the inductance value from $80 \mu \mathrm{H}$, at point $\mathbf{1}$, to $120 \mu \mathrm{H}$, at point $\mathbf{2}$, implies that the PS is required to increase as well, from $90^{\circ}$ to $50^{\circ}$, to achieve the same power level. The higher PS implies a larger time to charge or discharge the parasitic capacitances of the MOSFETs and thus, achieve ZVS again.

Fig. 34 further clarifies the idea by illustrating the measured ac primary and secondary bridge voltages, and the corresponding ac current in the inductor measured at primary and secondary sides of the transformer. It can be observed that during the operation at point 2 , the values of the currents $I_{0}$ and $I_{1}$ are positive, which is the initial condition for achieving ZVS, as discussed previously
Fig. 36. Experimental results. The measured ac primary and secondary bridge voltages (top), and the corresponding inductor current measured at the primary and secondary (bottom), for comparing the experimental operation at: (a) point 3 and (b) point 4

in Section II. However, on the other hand, during the operation at point 1 , the value of the current $I_{1}$ is negative, so the ZVS is not achieved in this case.

On the other hand, to compare points $\mathbf{3}$ and $\mathbf{4}$, Fig. 35 is generated, while additionally illustrating the boundaries for the reactive currents. It can be observed that point $\mathbf{3}$ presents an operation condition of the converter at high circulating currents, which exceeds the set boundary. This explains the low efficiency obtained when comparing to point 4 . Therefore, when moving to point 4 , as the inductance value is decreased from 80 to $73 \mu \mathrm{H}$, the PS is required to decrease as well, from $85^{\circ}$ at point 3 to $50^{\circ}$ at point 4 , to achieve the same power level. The lower PS angle implies a lower angle between the voltage and current applied to the transformer, thereby lower circulating currents, which brings the converter operation again within the boundaries of the reactive currents.

Fig. 36 further clarifies the idea by illustrating that the measured ac waveforms are similar to the previous case. The reactive current is marked by the red shaded areas on the plots. It can be observed that during the operation at point $\mathbf{3}$, the reactive current is higher than that occurring during the operation point 4. 
To conclude this discussion, it can be conceived that combining the PS angle with the inductance as two control parameters of the power flow in the DAB converter results in boosting the efficiency along the entire operation range.

\section{CONCLUSION}

A DAB converter using VI has been proposed. An electromagnetic model has been developed which describes the behavior of a VI using the reluctance equivalent circuit and including the device losses. Also, the VI was simulated within a DAB converter in a circuit-based time-domain simulation. Using these models, a new degree of freedom was explored for the control of power transfer in the DAB converter using VI, as well as studying the operational margins for combining both PS control with the variable inductance one. Moreover, the analysis opened several aspects for the improvement of DAB converter operation using the VI solution, such as extending the limits of the power transfer in the converter, linearization of the control function, and boosting the converter efficiency over a wider load range. The experimental results clarified the effect of the inductance variation on the power transfer in a DAB converter under maximum PS, and the overall efficiency of the proposed DAB exceeded the conventional converter by around 5\% at light and heavy loading conditions.

While it is true that the controlled saturation of the magnetic core allows handling a higher power with the same core size [18], however, at this point in the investigation it cannot be postulated, on an undisputed technical basis, that this also implies a reduction in the final size of the entire converter. To arrive at such a conclusion, the study herein can be extended to consider the extra size of the electronic components added for controlling the VI. Given the fact that these components should be rated for very small power, i.e., $<10 \mathrm{~W}$, therefore, it is quite feasible to optimize the size of the extra control converter [39]-[41]. It is also worth to note that scaling up the converter to higher power levels is not limited by the VI. The device can be designed for a higher power level by utilizing a core material which presents higher values of magnetic flux saturation, such as iron powder [14], [42]. Additionally, the VI design can be modified to allow higher inductance variation ranges.

As a future development, the shares of the losses among the different converter components can be studied and demonstrated for the full operation range.

\section{REFERENCES}

[1] H. Fan and H. Li, "High-frequency transformer isolated bidirectional DCDC converter modules with high efficiency over wide load range for 20 kVA solid-state transformer," IEEE Trans. Power Electron., vol. 26, no. 12, pp. 3599-3608, Dec. 2011.

[2] A. Burgio, D. Menniti, M. Motta, A. Pinnarelli, N. Sorrentino, and P. Vizza, "A laboratory model of a dual active bridge DC-DC converter for a smart user network," in Proc. IEEE 15th Int. Conf. Environ. Elect. Eng., Jun. 2015, pp. 997-1002.

[3] G. Oggier, G. O. García, and A. R. Oliva, "Modulation strategy to operate the dual active bridge DC-DC converter under soft switching in the whole operating range," IEEE Trans. Power Electron., vol. 26, no. 4, pp. 1228-1236, Apr. 2011.

[4] H. Bai and C. Mi, "Eliminate reactive power and increase system efficiency of isolated bidirectional dual-active-bridge DC-DC converters using novel dual-phase-shift control," IEEE Trans. Power Electron., vol. 23,no. 6, pp. 2905-2914, Nov. 2008.
[5] Y. Xie, J. Sun, and J. S. Freudenberg, "Power flow characterization of a bidirectional galvanically isolated high-power dc-dc converter over a wide operating range," IEEE Trans. Power Electron., vol. 25, no. 1,pp. 54-66, Jan. 2010.

[6] F. Krismer and J. W. Kolar, "Efficiency-optimized high-current dual active bridge converter for automotive applications," IEEE Trans. Ind. Electron., vol. 59, no. 7, pp. 2745-2760, Jul. 2012.

[7] F. Krismer and J. W. Kolar, "Accurate small-signal model for the digital control of an automotive bidirectional dual active bridge," IEEE Trans. Power Electron., vol. 24, no. 12, pp. 2756-2768, Dec. 2009.

[8] C. Calderon, A. Barrado, A. Rodriguez, A. Lazaro, C. Fernandez, and P. Zumel, "Dual active bridge with triple phase shift by obtaining soft switching in all operating range," in Proc. IEEE Energy Convers. Congr. Expo., 2017, pp. 1739-1744.

[9] J. Everts, "Closed-form solution for efficient ZVS modulation of DAB converters," IEEE Trans. Power Electron., vol. 32, no. 10, pp. 7561-7576, Oct. 2017

[10] S. Saeed, "Contributions to the design, operation and control of DAB bidirectional converters based on variable magnetic elements," Ph.D dissertation, 2020

[11] P. Garcia, S. Saeed, A. Navarro-Rodríguez, J. García, and H. Schneider, "Switching frequency optimization for a solid state transformer with energy storage capabilities," IEEE Trans. Ind. Appl., vol. 54, no. 6 , pp. 6223-6233, Nov./Dec. 2018

[12] Z. Qin, Y. Shen, P. C. Loh, H. Wang, and F. Blaabjerg, "A dual active bridge converter with an extended high-efficiency range by DC blocking capacitor voltage control," IEEE Trans. Power Electron., vol. 33, no. 7, pp. 5949-5966, Jul. 2018.

[13] A. Rodríguez, A. Vásquez, D. G. Lamar, M. M. Hernando, and J. Sebastian, "Different purpose design strategies and techniques to improve the performance of a dual active bridge with phase-shift control,' in Proc. IEEE 15th Workshop Control Model. Power Electron., Jun. 2014 pp. 1-10.

[14] M. S. Perdigão, M. Menke, A. R. Seidel, R. A. Pinto, and J. M. Alonso, "A review on variable inductors and variable transformers: Applications to lighting drivers," in Proc. IEEE Ind. Appl. Soc. Annu. Meeting, 2014, pp. 1-8.

[15] E. Orietti, P. Mattavelli, G. Spiazzi, C. Adragna, and G. Gattavari, "Twophase interleaved LLC resonant converter with current controlled inductor," in Proc. Braz. Power Electron. Conf., 2009, pp. 298-304.

[16] J. M. Alonso, M. S. Perdigao, D. Gacio, L. Campa, and E. S. Saraiva, "Magnetic control of DC-DC resonant converters provides constan frequency operation," Electron. Lett., vol. 46, no. 6, pp. 440-442, Mar. 2010.

[17] M. S. Perdigão, B. Baptista, H. Marques, E. S. Saraiva, and J. M. Alonso, "On the use of magnetic regulators in electronic ballasts," in Proc. 45th Int. Univ. Power Eng. Conf., 2010, pp. 1-6.

[18] M. S. Perdigão, J. Pedro F. Trovão, J. M. Alonso, and E. S. Saraiva, "Largesignal characterization of power inductors in EV bidirectional DC-DC converters focused on core size optimization," IEEE Trans. Ind. Electron., vol. 62, no. 5, pp. 3042-3051, May 2015.

[19] F. Krismer, S. Round, and J. W. Kolar, "Performance optimization of a high current dual active bridge with a wide operating voltage range," in Proc. 37th IEEE Power Electron. Spec. Conf., 2006, pp. 1-7.

[20] A. Rodríguez, J. Sebastian, D. G. Lamar, M. M. Hernando, and A. Vazquez, "An overall study of a dual active bridge for bidirectional DC/DC conversion," in Proc. IEEE Energy Convers. Congr. Expo., Sep. 2010, pp. 1129-1135.

[21] A. R. Alonso, "Análisis de arquitecturas multipuerto para la alimentación de sistemas electrónicos con múltiples flujos de energía," Ph.D. dissertation, Dept. Elect. Eng., Univ. Oviedo, Oviedo, Spain, 2015. [Online]. Available: http://hdl.handle.net/10651/20286

[22] S. Saeed, "Design and construction of an isolated DC to DC switching converter for integration of energy storage systems in power electronic applications," M.S. thesis, Dept. Elect. Eng., Univ. Oviedo, Oviedo, Spain, 2015. [Online]. Available: http://hdl.handle.net/10651/33257

[23] J. M. Alonso, G. Martínez, M. Perdigão, M. Cosetin, and R. N. do Prado, "Modeling magnetic devices using SPICE: Application to variable inductors," in Proc. IEEE Appl. Power Electron. Conf. Expo., 2016, pp. 1115-1122.

[24] S. Saeed, J. García, and R. Georgious, "Modeling of variable magnetic elements including hysteresis and eddy current losses," in Proc. IEEE Appl. Power Electron. Conf. Expo., 2018, pp. 1750-1755.

[25] D. Medini and S. Ben-Yaakov, "A current-controlled variable inductor for high frequency resonant power circuits," in Proc. IEEE Appl. Power Electron. Conf. Expo., 1994, pp. 219-225. 
[26] S. Saeed, R. Georgious, and J. Garcia, "Modeling of magnetic elements including losses-Application to variable inductor," Energies, vol. 13, no. 8, Apr. 2020, Art. no. 1865, doi: 10.3390/en13081865.

[27] ROHM. Datasheet of SiC MOSFET SCT2280KE. [Online]. Available: https://fscdn.rohm.com/en/products/databook/datasheet/discrete/sic/ mosfet/sct2280ke-e.pdf

[28] J. G. Tiemblo, "Investigations on reactive power and dead time compensation for a double active bridge with a planar transformer," Master's thesis, Univ. Oviedo, Oviedo, Spain, 2014. [Online]. Available: http://hdl.handle.net/10651/28158

[29] Texas Instruments ${ }^{\mathrm{TM}}$, TMS320F28335 digital signal controller. [Online]. Available: http://www.ti.com/lit/ds/symlink/tms320f28335.pdf?ts= 1588453618532

[30] Texas Instruments, Code Composer Studio integrated development environment for c2000 microcontrollers. [Online]. Available: http: //www.ti. $\mathrm{com} /$ tool/CCSTUDIO

[31] E. Cardelli, L. Fiorucci, and E. D. Torre, "Estimation of MnZn ferrite core losses in magnetic components at high frequency," IEEE Trans. Magn., vol. 37, no. 4, pp. 2366-2368, Jul. 2001.

[32] D. C. Jiles and D. L. Atherton, "Ferromagnetic hysteresis," IEEE Trans. Magn., vol. 19, no. 5, pp. 2183-2185, Sep. 1983.

[33] W. and Peter Reid, "Modelling and simulation of magnetic components in electric circuits," Ph.D. dissertation, Dept. Elect. Comput. Eng., Univ. Southampton, Southampton, U.K., Nov. 2001. [Online]. Available: https: //eprints.soton.ac.uk/368484/

[34] P. L. Dowell, "Effects of eddy currents in transformer windings," Proc. Inst. Elect. Eng., vol. 113, no. 8, pp. 1387-1394, Aug. 1966.

[35] A. Massarini and M. K. Kazimierczuk, "Self-capacitance of inductors," IEEE Trans. Power Electron., vol. 12, no. 4, pp. 671-676, Jul. 1997.

[36] B. Zhao, Q. Song, W. Liu, and Y. Sun, "Dead-time effect of the high frequency isolated bidirectional full-bridge dc-dc converter: Comprehensive theoretical analysis and experimental verification," IEEE Trans. Power Electron., vol. 29, no. 4, pp. 1667-1680, Apr. 2014

[37] M. Kim, M. Rosekeit, S. Sul, and R. W. A. A. De Doncker, "A dualphase-shift control strategy for dual-active-bridge DC-DC converter in wide voltage range," in Proc. 8th Int. Conf. Power Electron. - ECCE Asia, 2011, pp. 364-371.

[38] H. Fan and H. Li, "High-frequency transformer isolated bidirectional DCDC converter modules with high efficiency over wide load range for 20 kVA solid-state transformer," IEEE Trans. Power Electron., vol. 26, no. 12, pp. 3599-3608, Dec. 2011.

[39] M. W. Beraki, J. P. F. Trovão, M. S. Perdigão, and M. R. Dubois, "Variable inductor based bidirectional DC-DC converter for electric vehicles," IEEE Trans. Veh. Technol., vol. 66, no. 10, pp. 8764-8772, Oct. 2017.

[40] M. Beraki, M. Perdigao, F. Machado, and J.P. Trovão, "Auxiliary converter for variable inductor control in a DC-DC converter application," in Proc. 51st Int. Univ. Power Eng. Conf., 2016, pp. 1-6.

[41] J. M. Alonso, M. S. Perdigão, M. A. D. Costa, G. Martínez, and R. Osorio, "Analysis and experiments on a single-inductor half-bridge LED driver with magnetic control," IEEE Trans. Power Electron., vol. 32, no. 12, pp. 9179-9190, Dec. 2017.

[42] Y. Liu, H. A. Mantooth, J. C. Balda, and C. Farnell, "Realization of high-current variable AC filter inductors using silicon iron powder magnetic core," in Proc. IEEE Appl. Power Electron. Conf. Expo., 2017, pp. 855-860.

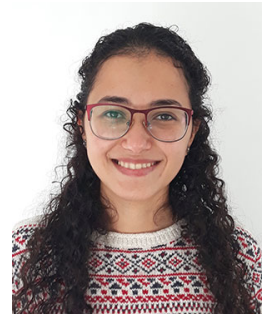

Sarah Saeed (Student Member, IEEE) received the B.Sc. degree in electrical engineering from Ain Shams University, Egypt, in 2013, and the M.Sc. degree in electrical energy conversion and power systems and the $\mathrm{Ph} . \mathrm{D}$. degree in energy and process control, both from the University of Oviedo, Spain, in 2015 and 2020, respectively.

She is a Postdoctoral Researcher with Lemur Research Group, University of Oviedo, Oviedo, Spain. She has coauthored five journal papers and 15 international conferences. Her research interests include the modeling and control of power electronic converters, design and construction of $\mathrm{dc} / \mathrm{dc}$ converter topologies, integration of energy storage systems in power electronic applications, and nonlinear magnetics.

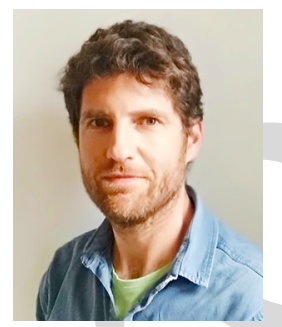

Jorge Garcia (Senior Member, IEEE) received the M.Sc. and Ph.D. degrees in electrical engineering from the University of Oviedo, Gijon, Spain, in 2000 and 2003, respectively.

In December 1999, he joined the Electrical and Electronic Engineering Department, University of Oviedo, where he is currently an Associate Professor. $\mathrm{He}$ is a Founding Member of the LEMUR Research Team. He has coauthored more than 50 journal papers and more than 100 international conference papers in power and industrial electronics. His research interests include analysis, modeling, and design of power electronics and control stages for industrial, grid support, and lighting applications.

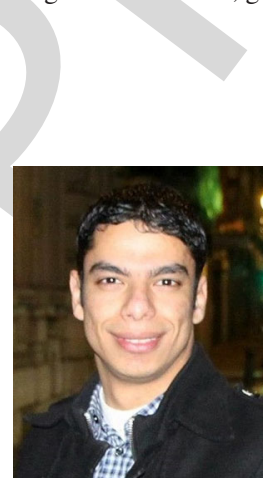

Ramy Georgious (Member, IEEE) received the B.Sc. degree in electrical engineering from Port Said University, Port Said, Egypt, in May 2010, and the M.Sc. degree in electrical energy conversion and power systems and the Ph.D. degree in electrical engineering from the University of Oviedo, Oviedo, Spain, in 2014 and 2018, respectively.

In July 2014, he joined LEMUR Research Team, University of Oviedo, Gijon, Spain. In 2015, he was a Visitor Researcher with Power Electronics, Machines, and Control Research Group, University of Nottingham, Nottingham, U.K. He is currently an Assistant Professor with the Department of Electrical, Computer, and Systems Engineering, University of Oviedo, and with the Electrical Engineering Department, Port Said University, and also a Director of the Hardware Research and Development Department, ENFASYS Engineering Company, Gijon, Spain. His research interests include energy storage systems, control systems, power electronic converters, microgrids, renewable energy, design, and simulation.
1081 1082 1083 1084 1085 1086 1087 1088 1089 1090 1091 1092 1093 1094 1095

1096 1097 1098 1099 1100 1101 1102 1103 1104 1105 1106 1107 1108 1109 


\title{
Dual-Active-Bridge Isolated DC-DC Converter With Variable Inductor for Wide Load Range Operation
}

\author{
Sarah Saeed ${ }^{\circledR}$, Student Member, IEEE, Jorge Garcia ${ }^{\circledR}$, Senior Member, IEEE, \\ and Ramy Georgious $\mathbb{D}^{\mathbb{D}}$, Member, IEEE
}

\begin{abstract}
This article explores the use of a variable inductor as a reactive element for energy transfer in a dual-active-bridge (DAB) converter. By using a controlled variable inductor, the optimal switching region in the operation of the phase-shift DAB can be extended, and thus, high efficiencies can be achieved over wider load ranges compared to the traditional solution. Moreover, the combined use of the variable inductance together with the phase shift, as two control parameters, allows for the linearization of the DAB converter transfer function, which improves the stability and the performance of the controller. And finally, due to the controlled saturation of the device, it is feasible to reduce the magnetic core size, yielding a size optimization of the full converter. This article develops a circuit-based model to simulate the converter system, aiming to study the proposed improvements. Furthermore, a 2-kW $\mathrm{SiC}$-based DAB converter prototype is constructed, including a controlled variable inductor. The experimental results presented in this article validate the studies carried out for the different operation conditions.
\end{abstract}

Index Terms-Dual-active-bridge, efficiency, hysteresis losses, variable magnetics, zero voltage switching. HE electrical utility network of today is facing a significant change driven by the need for improved efficiency, reliability, and safety. To achieve these requirements, there is a growing interest toward the smart grid systems which incorporate different systems using power electronics technologies. The most important of these systems is the solid-state transformer (SST)

Manuscript received October 12, 2020; accepted December 18, 2020. This work was supported in part by the European Union's H2020 Research and Innovation Programme under Grant 864459 (UE-19-TALENT-864459), in part by the Spanish Government (Innovation Development and Research Office-MEC) under Research Grant ENE2016-77919, "Conciliator" Project, "B2B" Project Nuevas vías hacia la gestión descentralizada de la energía de edificio a edificio PID2019-111051RB-100, and "Torres Quevedo" Grant PTQ2019-010579, and in part by the Government of the Principality of Asturias under Grant FC-GRUPIN-IDI/2018/000241, and in part by the "Severo Ochoa" Program of Predoctoral Grants for Training in Research and University Teaching under Grants PA-17-PF-BP16-133 and PA-13-PF-BP13-138. Recommended for publication by Associate Editor R. Ayyanar. (Corresponding author: Sarah Saeed.)

Sarah Saeed and Jorge Garcia are with the Lemur group, Electrical Engineering Department, University of Oviedo, 33204 Gijon, Spain (e-mail: saeedsarah@uniovi.es; garciajorge@uniovi.es).

Ramy Georgious is with the Lemur group, Electrical Engineering Department, University of Oviedo, 33203 Gijon, Spain, and also with the Electrical Engineering Department, Port Said University, Port Said 42526, Egypt (e-mail: eng.ramy.georgious@ @otmail.com).

Color versions of one or more figures in this article are available at https: //doi.org/10.1109/TPEL.2020.3048928.

Digital Object Identifier 10.1109/TPEL.2020.3048928 which can radically change the utility power distribution by more efficient routing of power, voltage regulation, and flexibility. The basic and most popular structure of the SST is the dual-active bridge (DAB). DAB converter allows bidirectional power flow by means of regulating the phase shift (PS) between the converter bridges. However, this modulation scheme can achieve high efficiencies only at voltage levels that match the transformer turns ratio; significant mismatches lead to transformer RMS currents, inductor and transformer copper losses, and increased semiconductor conduction losses [1]-[3]. Also, circulating currents take place due to the PS between the voltage and the current applied to the transformer [4]. Another limitation is the loss of zero-voltage switching (ZVS) at light loading conditions [5], [6]. For tackling those limitations, many alternative modulation schemes have been proposed in [3], [4], and [6]-[8]. As the complexity of the modulation scheme increases, it compromises the ease and time of implementation of the control function [9].

Another approach to tackle the limitations of the DAB operation is to use the variation in the switching frequency as an alternative parameter to control the power flow in the converter. However, the frequency variation has a significant effect on the converter efficiency specifically in the presence of energy storage systems (ESS) [10], [11]. The switching frequency variation range is also limited by the design of magnetic components, namely, the series inductor and the high-frequency (HF) transformer. Finally, it is not straightforward to change the frequency online during the converter operation since it implies a change in the sampling period of the programmer, as well as the interrupt periods for acquiring correct measurements of the sensors data. Consequently, to widen the soft-switching range and reduce circulating currents, most of the literature works have explored modulation strategies which maintained a fixed switching frequency [12]. To this motivation, the inductance variation, achieved throughout the use of variable inductors (VI), is proposed in this work as a promising control parameter of the power flow in the DAB converter [13].

VI is an electromagnetic device that is based on changing the inductive impedance of a winding placed around a saturable magnetic core [14]. This mechanism is achieved by changing the saturation level of the magnetic core [14]. These devices, therefore, allow for additional degrees of freedom in the design and control of power electronic converters (PECs) [14]. This is particularly useful in resonant converters, where the output power is generally controlled by varying the switching frequency due to the power versus frequency characteristic of 
the converter [15], [16]. This standard frequency control presents some drawbacks due to electromagnetic interference (EMI) issues, complex filtering, or variable sampling time, among others, especially for a wide range of variation. However, if variable magnetics are used, the same control margins can be obtained at a constant switching frequency, therefore, allowing for an optimization of the EMI filters and sampling procedures [14], [17].

Also for $\mathrm{dc}-\mathrm{dc}$ bridge converters, such as the DAB or the dual-half-bridge (DHB), the addition of a new degree of freedom to the control scheme by means of the VI can enhance the operational performance of the system, for instance, by increasing the soft-switching margins, as reported in preliminary works [1], [2]. Specifically, in [1], where the VI concept was proposed as the main energy transfer element for DHB converter, the study concluded that the ZVS operation region of such topologies is controlled by means of PS control by employing an adaptive inductor. Therefore, high efficiency can be achieved over wider load ranges compared to the conventional converter. On the other hand, for the DAB converter, the authors in [2] adopted a solution based on inserting an additional series inductor at light load conditions, aiming to decrease circulating currents and thus, allowing for an increase in the converter efficiency. Consequently, these studies reinforce the idea of introducing an adaptive power transfer inductor in the DAB converter to reduce the overall power losses. However, the solution proposed in [2] is based on mechanical switching of a combination of fixed inductance values. The obvious benefits of mechanical switching are the simplicity and low cost. However, it allows only stepwise control, and it is too slow to provide a stable operation. On the other hand, the magnetically controlled VI allows continuous variation of the inductance; therefore, it will be used in the proposed study herein.

The improvement of the DAB converter efficiency is not the only feature that the VI can provide, there are several potential features that can be explored. Another feature is the use of the inductance variation to linearize the control-to-output transfer function. This has implications in the static gain characteristic, which can allow several advantages to the converter control system. For example, in solar converters, the maximum power point tracking (MPPT) technique can be smoother without introducing dithering or instability due to nonlinear power flow characteristics.

Another potential improvement that the VI can add to the DAB converter is the possibility to extend the maximum power level, which results from decreasing the inductance as a function of the PS to allow linear phase-to-power transfer function. This implies that, by means of controlled saturation of the magnetic core, the same magnetic core size can be used for a higher power level. This could allow optimizing the size of the magnetic element. However, the possibility to boost the converter power density by using VI is limited by the additional volume and losses of the power electronic devices which are needed for controlling the magnetic core saturation [18].

Moreover, it is worth to notice that replacing the fixed power transfer inductance by a variable one, the dynamic performance of the control scheme can also be modified. In fact, it is

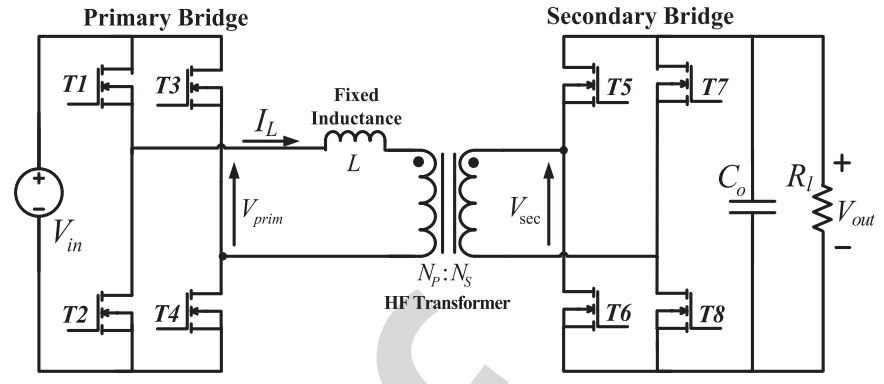

Fig. 1. Schematic of DAB converter

postulated that regarding the disturbance rejection upon closedloop control of the converter, the adaptive inductor adds an extra control parameter, which can be employed to assist the main control scheme in riding through the transient event. In all cases, it is necessary to investigate the overall PEC figures of merit incorporating the VI.

The aim of this article is to study the potential enhancements to the operation of the DAB converter by using a VI. The focus of this article is to present the improvements in the converter efficiency under light loads (by extending the ZVS range) and heavy loads (by reducing circulating currents), as well as the linearization of the power flow characteristics, and finally, the improvement of the dynamic response of the controlled system.

The analysis is carried out using an electromagnetic system simulation model which takes into consideration different component losses, such as switching and conduction losses, magnetic device core and winding losses, etc. In this context, Section II states the theoretical background that discusses the structure and the principle of operation of the conventional DAB converter. Also, the latter section provides a briefing about the VI structure, operation, and model. Section III explains the models for different system devices. The models are adjusted with the electrical characteristics of the test setup, in order to develop real simulations and particularize the analysis for the constructed prototype. In Section IV, several potential aspects are studied for extending the operation range of the DAB converter by using a VI. In Section V, the test setup and the experimental results are presented. Finally, Section VI concludes the article.

\section{PROPOSED DAB USING VI}

\section{A. Principle of Operation of Conventional DAB Converter}

The DAB converter is a bidirectional isolated dc-dc power conversion topology which is composed of two full-bridge inverter circuits, interfaced across a constant power transfer inductor that usually includes an HF transformer, as clarified by Fig. 1 .

The flexibility of the primary and secondary active full bridges in this topology allows for various modulation methods to minimize the losses and to maximize the efficiency over a wide load and voltage operation ranges [6], [19]. Using the simplest modulation technique, named single-PS (SPS) scheme, the full-bridge converters are operated at a constant switching frequency, with $50 \%$ maximum duty cycle. After a thorough 


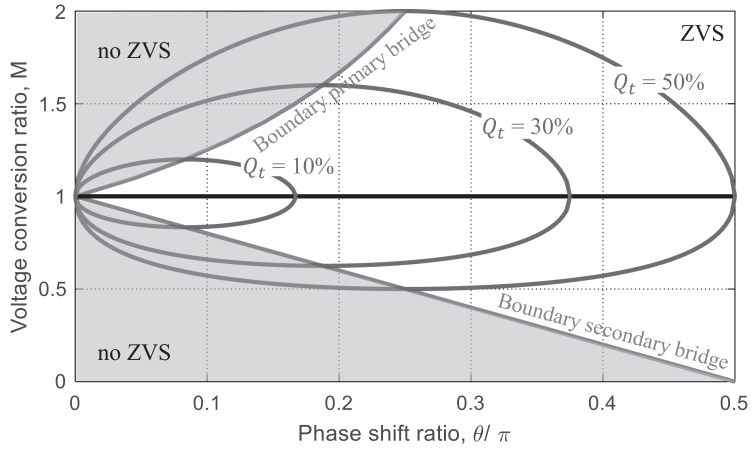

Fig. 2. ZVS boundaries of the DAB converter.

study of the circuit operation, it can be demonstrated that the bridge leading by a phase angle sends power to the lagging one [20]. The analytical expression for the power flow in the converter under SPS modulation scheme is

$$
P_{\mathrm{DAB}}=\frac{V_{\text {in }} V_{\text {out }}}{X_{L} r_{t}} \theta\left(1-\frac{\theta}{\pi}\right) .
$$

From (1), the control parameters that affect the power transfer are the input and output voltage levels, $V_{\text {in }}$ and $V_{\text {out }}$, respectively, the transformer turns ratio $r_{t}=N_{s} / N_{p}$, the PS between the primary and secondary bridges $\theta$, and the impedance $X_{L}=2 \pi f L$, $f$ being the switching frequency, and $L$ the inductance of the coil.

In case of having a constant voltage source at the output of the DAB converter, then the wide-voltage and wide-load are distinctive terms that need to be handled carefully. The voltage range indicates the relationship between the input and output voltage levels, while the loading condition indicates the amount of power demanded either by the input or the output sources, i.e., in forward or reverse direction. On the other hand, in the case of having a resistive load at the output, the resistance value determines the voltage level for a certain amount of demanded power, in this case, only to the resistive load, i.e., in the forward direction. In this latter case, the signification of the wide-voltage and wide-load terms is the same and can be interchangeable.

\section{B. Limitations of the Converter Operation Under Wide-Voltage and Wide-Load Ranges}

To achieve ZVS in the converter switching devices, the inductor current should be enough to charge and discharge the parasitic output capacitors of the switches [21]. Analyzing the inductor current waveform in analogy with the switching waveforms at both bridges, the ZVS conditions for the primary and the secondary bridges can be formulated in terms of the voltage conversion ratio $M$ and the PS ratio $\theta / \pi$ as explained in detail in [13], [20], and [21]. The ZVS boundaries of the DAB converter can thus be represented as shown in Fig. 2.

It can be observed that, ideally, for $M=1$, ZVS can be achieved over the full range of operation. For values of $M$ different than 1, the PS must be large enough to provide the required energy for achieving the ZVS condition. Operation under small PSs, i.e., upon low load conditions, is thus limited by the ZVS range, which implies that the converter should be designed to operate at the maximum PS in order to allow higher values of current and facilitate ZVS.

On the other hand, higher values of PS imply that the phase angle between the current and the voltage applied to one side of the HF transformer increases as well. Consequently, the reactive circulating currents are higher. Those currents result in an increase in the conduction losses and elevate the reactive power losses [4], [22] which eventually cause a drop in the converter efficiency. To emphasize the effect of the PS on the reactive currents, the total percentage reactive currents in the converter is formulated again in terms of $M$ and $\theta / \pi$, as explained in detail in [21].

It can be concluded that under mismatches in the voltage levels $(M \neq 1)$, the soft-switching range is significantly constrained, which limits the operation of the converter at low PS angles. Additionally, since the PS angle between the bridges is the only control parameter, therefore, under higher PS angles, the reactive currents are higher.

Consequently, to widen the ZVS range and reduce circulating currents, most of the literature has explored new modulation strategies other than the SPS. Those alternative modulation schemes consist in introducing extra degrees of freedom to control the power transfer. This is achieved by inserting an extra PS angle between the modulation pulses of two legs in a full bridge [3], [4], [6]-[8]. Therefore, the resulting ac voltages will be three-level with a zero-voltage period during the switching transitions, which allows increasing ZVS and reducing circulating currents. However, the increase in the number of variables, introduced by these modulation schemes, results in cumbersome and time-consuming computations which might restrict the resources of digital processors in real-time programming [9].

This article hereafter proposes a different solution instead of changing the operational margins, that is, to change the operation point of the converter itself. This can be carried out by introducing another control parameter, which is the inductance variation in combination with the PS angle. From (1), it is clear that replacing the fixed inductor by a variable one can serve as an extra control parameter for the power transfer in the converter.

\section{VI: Structure and Operation}

From the study of the state of the art regarding possible physical structures to implement the VI, the double E-core setup, depicted in Fig. 3(a), is considered to be the most appropriate and most comprehend in the literature [23]-[25]. The structure consists of two E-cores stacked on top of each other to form three magnetic arms with an air gap in the center arm. The main winding is wound around the center arm, and the control windings are wound around the lateral left and right arms; they are serially connected in opposite polarity so as to cancel out the ac voltages induced by the center leg [25].

In this arrangement, the current flowing through the main winding $N_{c}$ generates an ac flux $\phi_{C}$ which circulates through the center arm and splits to the outer left and right arms. Applying a relatively small dc current $I_{b}$ to the bias control windings $N_{b}$, a dc flux is produced in the right $\operatorname{arm} \phi_{R}$ and the left $\operatorname{arm} \phi_{L}$, which tends to circulate mainly through the outer, ungapped, 


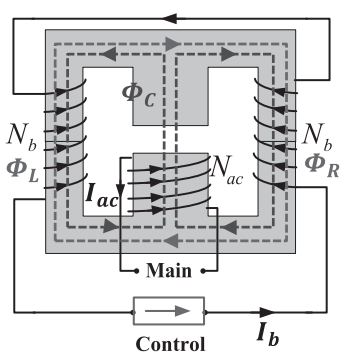

(a)

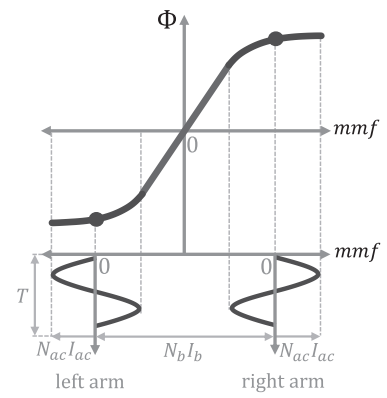

(b)

Fig. 3. Variable inductor based on double E-core structure. (a) Structure of the device and windings. (b) Operation of VI in nonlinear region of $B(H)$ curve.

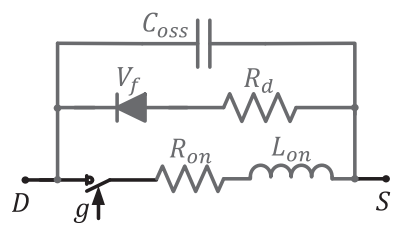

Fig. 4. SiC MOSFET model.

circumference of the core [25]. The dc flux will bias the operation point of the magnetic material within the $B(H)$ curve toward the nonlinear region, causing the inductance seen from the main winding terminals to vary as a function of the dc bias current [23], [26]. The operation is clarified by Fig. 3(b), which shows the operation points on the $B(H)$ curve for the left and right arms. The constant dc control current will produce a constant $m m f$ in the left and right arms, which will bring these sections into the nonlinear region of the magnetization curve.

\section{EleCtromagnetic System Model}

The following paragraphs aim to model the full system, which includes the DAB converter, the VI, and the HF transformer. Also, specific details and electrical characteristics of the devices used in the constructed prototype are added to the model. This will allow an accurate representation of the full system in order to analyze the performance of the converter when the conventional inductor is replaced by a variable one.

\section{A. Power Switches Model}

To account for the switching and conduction losses, the $\mathrm{SiC}$ MOSFETS are modeled as shown by the circuit diagram in Fig. 4. Specific to this application, the SCT2280KE devices are used [27], with the characteristic parameters indicated in Table I.

\section{B. HF Transformer Model}

The transformer used for the study is an HF planar transformer. The specifications and ratings of the transformer are stated in Table I. To characterize the transformer, the classic transformer circuit is used. The measurements and characterization were done as part of previous works [22], [28]. Nevertheless, a summary of the procedure and the obtained parameters are presented hereafter for convenience.
TABLE I

SPECIFICATIONS OF DIFFERENT DEVICES

\begin{tabular}{|c|c|c|}
\hline \multirow{6}{*}{$\begin{array}{l}\text { Power } \\
\text { Switch }\end{array}$} & Commercial Reference & SCT2280KE [27] \\
\hline & ON Resistance, $R_{o n}$ & $280 \mathrm{~m} \Omega$ \\
\hline & ON Inductance, $L_{o n}$ & $2.5 \mu \mathrm{H}$ \\
\hline & Diode Resistor, $\boldsymbol{R}_{d}$ & $300 \mathrm{~m} \Omega$ \\
\hline & Forward Voltage, $V_{f}$ & $4 \mathrm{~V}$ \\
\hline & Output Capacitance, $C_{\text {oss }}$ & $27 \mathrm{pF}$ \\
\hline \multirow{10}{*}{$\begin{array}{c}\text { HF } \\
\text { Transformer }\end{array}$} & Commercial Reference & Himag planar 500 \\
\hline & Rated power & $10 \mathrm{~kW}$ \\
\hline & Transformer Ratio, $N_{p} / N_{s}$ & $18: 22$ \\
\hline & Primary Series Resistance, $\boldsymbol{R}_{s p}$ & $0.2 \Omega$ \\
\hline & Primary Series Inductance, $L_{s p}$ & $2.5 \mu \mathrm{H}$ \\
\hline & Secondary Series Resistance, $\boldsymbol{R}_{s s}$ & $0.2 /(22 / 18)^{2} \Omega$ \\
\hline & Secondary Series Inductance, $L_{s s}$ & $2.5 /(22 / 18)^{2} \mu \mathrm{H}$ \\
\hline & Magnetizing Inductance, $L_{m}$ & $7.044 \mathrm{mH}$ \\
\hline & Magnetizing Resistance, $\boldsymbol{R}_{m}$ & $188.2 \mathrm{k} \Omega$ \\
\hline & Magnetizing Capacitance, $C_{m}$ & $0.2388 \mathrm{nF}$ \\
\hline \multirow{5}{*}{$\begin{array}{l}\text { Variable } \\
\text { Inductor }\end{array}$} & Magnetic Core Size & ETD49/25/16 \\
\hline & Magnetic Core Material & N87 \\
\hline & Main winding no. of Turns, $N_{c}$ & 23 turns \\
\hline & Bias winding no. of Turns, $N_{b}$ & 55 turns \\
\hline & Inductance Range & $70-140 \mu \mathrm{H}$ \\
\hline
\end{tabular}

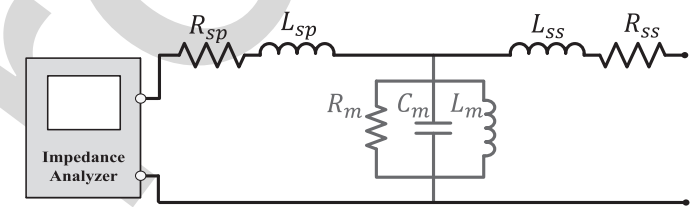

Fig. 5. No-load test to estimate magnetizing impedances of the transformer.

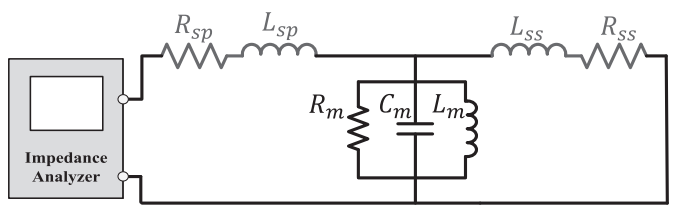

Fig. 6. Short-circuit test to estimate the series impedances of the transformer.

To determine the characteristic parameters of the transformer, the conventional method is used which consists in performing open and short-circuit tests on the transformer windings. During the open-circuit test, also referred to as the no-load test, the impedance analyzer is connected to the terminals of the primary winding with the secondary winding open-circuited. Fig. 5 explains the process. The objective of this test is to estimate the magnetizing impedance of the transformer. Table I states the values of the obtained parameters. On the other hand, the short-circuit test was carried out by connecting the impedance analyzer to the primary winding with the secondary winding terminals short-circuited, as shown in Fig. 6. The objective of this test is to estimate the series inductance and resistance of primary and secondary windings. It is worth to note that the magnetizing impedance is much higher than that of the leakage path; therefore, the parallel magnetization branch can be neglected in the calculation. Table I states the values of the obtained parameters. Also, Fig. 7 illustrates the transformer circuit model along with the Bode plots illustrated in Fig. 8 . 


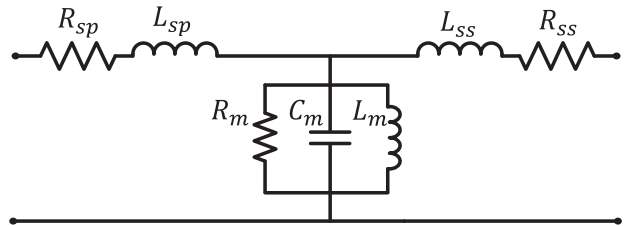

Fig. 7. HF transformer impedance model.

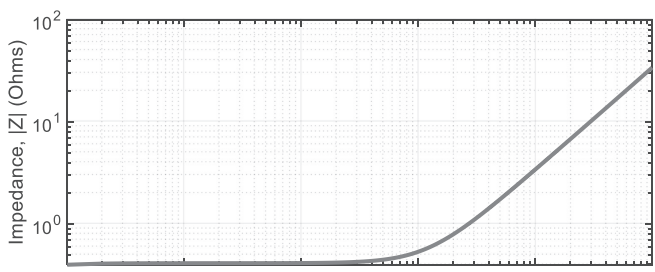

(a)

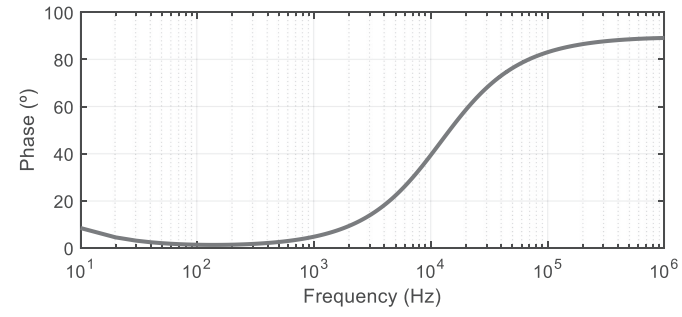

(b)

Fig. 8. Bode plots of transformer impedance model illustrating the (a) amplitude and (b) angle.

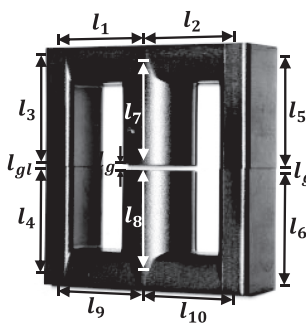

(a)

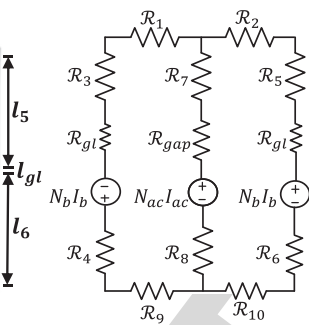

(b)

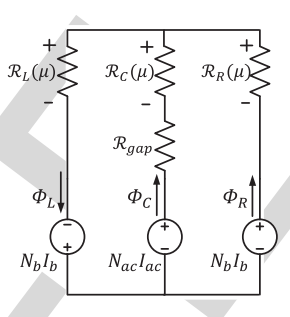

(c)

Fig. 9. Reluctance circuit of double E-core VI shown in Fig. 3. (a) Lengths of reluctance paths. (b) Corresponding circuit model. (c) Reluctance equivalent circuit.

\section{VI Model}

The VI is modeled based on the reluctance equivalent circuit [23], [24]. The magnetic core is modeled using three main elements. First, the constant reluctance, which models the nonferromagnetic material, such as the air gap, $\mathcal{R}_{\text {gap }}$. Second, the variable reluctance, which models the nonlinear behavior of ferromagnetic material, such as the left, right, and center arms, represented by $\mathcal{R}_{C}(\mu), \mathcal{R}_{L}(\mu)$, and $\mathcal{R}_{R}(\mu)$, respectively. And finally, the magnetomotive source, which models the winding electrical and magnetic interaction, such as the control windings represented by the duplicated sources $N_{b} . I_{b}$, and the main winding represented by $N_{c} . I_{c}$. The equivalent reluctance circuit model corresponding to the double-E core VI is illustrated in Fig. 9.

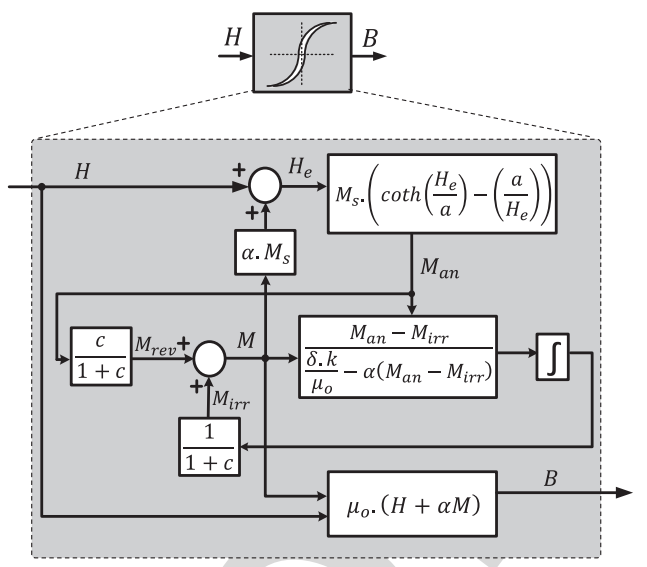

Fig. 10. Schematic of the implementation of JA model.

Therefore, the inductance can be calculated based on the reluctances of different paths in the magnetic circuit, as shown by

$$
L=\frac{N_{\mathrm{ac}}{ }^{2}}{\mathcal{R}_{C}(\mu)+\mathcal{R}_{\text {gap }}+\mathcal{R}_{L}(\mu) / / \mathcal{R}_{R}(\mu)} .
$$

The fully developed model is described in detail in previous works by the authors [24], [26]. The latter model takes into consideration a detailed description and modeling of the device losses, mainly core losses and winding eddy current losses. The necessary highlights of this loss model are presented below.

1) Model of Core Losses: The core losses in a magnetic device are divided into hysteresis loss and eddy currents loss. Previous studies in the literature [21] have shown that for a ferrite magnetic material operating in a range of frequency up to $100 \mathrm{kHz}$, the eddy current losses are a very small part of the total core losses. Therefore, for the range of frequencies under study herein, the eddy current losses in the magnetic core will be neglected for simplicity.

To calculate the hysteresis losses, the Jiles-Atherton (JA) hysteresis model is used [31]. The main strengths of the JA model compared to its counterpart models are — being the most suitable for development from a circuital simulation perspective, besides having good convergence, and acceptable accuracy among a variety of materials and operation conditions [33].

Fig. 10 shows a block diagram of the detailed implementation of the JA equations [26]. The parameters of the model are mainly five: $M_{s}, a, k, c$, and $\alpha$, which are initially estimated by an iterative procedure to fit the model to the magnetic material $B(H)$ curve data provided by the manufacturer [26]. Following this block diagram, the model equations have been implemented in Simulink. Therefore, for a given core size and magnetic material, the instantaneous magnetic flux density $(B)$ can be estimated for a certain instantaneous magnetic field intensity $(H)$ applied to the magnetic core.

2) Model of Winding Losses: The winding losses in a magnetic component are due to the resistance of the copper wire. At dc operation, or current waveforms of relatively low frequencies, this resistive component $R_{\mathrm{dc}}$ can be considered constant, and is a function of the resistivity of copper material, the temperature and 


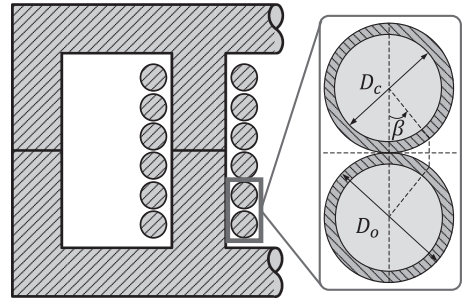

(a)

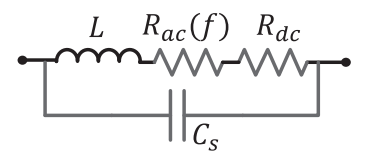

(b)

Fig. 11. Variable inductor winding model. (a) Configuration of the windings. (b) Full circuit model.

the geometry of the wire. However, as the switching frequency increases, two effects start to appear, which affects the resistance, the skin effect and proximity effect, both caused by eddy currents induced in the windings. These two effects alter the resistance of the winding and significantly contribute to the overall winding losses. It is necessary in this case to calculate the ac resistance of the winding.

Dowell provided a method that computes the equivalent winding resistance using a 1-D analytical approach [34]. It estimates the ac resistance $R_{\mathrm{ac}}$ by scaling the dc winding resistance $R_{\mathrm{dc}}$ by a factor

$$
R_{\mathrm{ac}}=R_{\mathrm{dc}}\left(M^{\prime}+\frac{\left(m^{2}-1\right) D^{\prime}}{3}\right)
$$

where $M^{\prime}$ and $D^{\prime}$ are coefficients defined based on the geometrical dimensions of the winding, material characteristics, and frequency of operation, and $m$ is the number of layers.

In order to attain a full, comprehensive model of the device, the stray capacitance of the winding is added to the model. The calculation of the stray capacitance is based on an analytical approach that divides the winding into partitions [35]. It considers turn-to-turn and turn-to-core capacitances of the inductor. The overall total stray capacitance of the coil converges to the expression: $C_{s} \cong 1.366 C_{t t}$, where $C_{t t}$ is the turn-to-turn capacitance of the coil, defined by

$$
C_{t t}=\varepsilon_{0} l_{t}\left[\frac{\varepsilon_{r} \beta^{*}}{\ln \left(D_{o} / D_{c}\right)}+\cot \left(\frac{\beta^{*}}{2}\right)-\cot \left(\frac{\pi}{12}\right)\right]
$$

where $l_{t}$ is the turn length, $\beta^{*}$ is the angular coordinate, $\varepsilon_{0}$ and $\varepsilon_{r}$ are the permittivity of air and relative permittivity of the insulation medium, respectively, and $D_{0}$ and $D_{C}$ are the diameters of the wire with and without the insulation coating, respectively. The VI winding configuration is illustrated in Fig. 11(a).

Also, the full impedance model of the winding in the magnetic device is expressed by the circuit diagram in Fig. 11(b).

It is worth to note that the design of a VI for handling an ac high-frequency main current, as the case of the DAB converter in context, is different in two aspects specifically; first, the winding losses in the device will be different. The absence

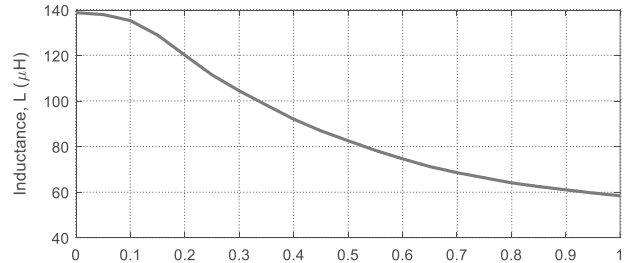

(a)

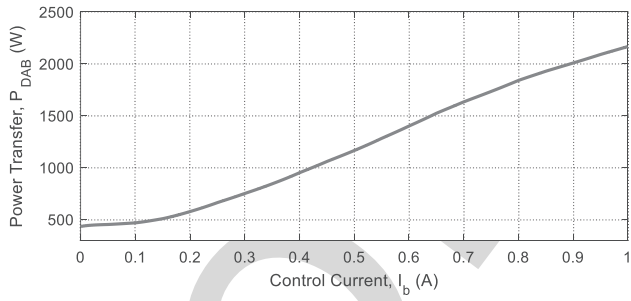

(b)

Fig. 12. Effect of inductance variation on power transfer in the proposed DAB converter. (a) Inductance as a function of bias current. (b) RMS power transfer in $\mathrm{DAB}$ converter as a function of bias current.

of a dc component in the current will imply lower dc power losses, while, on the other hand, a higher frequency current waveform would contribute to increase the eddy current losses due to skin and proximity effects. And second, the ac current implies that an induced voltage could be reflected from the ac main winding and generated across the dc control windings which would drastically affect the design of those windings to be able to handle higher levels of induced voltage. This, therefore, constrains the arrangement of the VI windings such that the two dc control windings be serially connected in opposite polarity to cancel the induced voltages due to the ac main winding.

In conclusion, once the significant elements of the electromagnetic circuit are defined, the developed model can thus be used to study the full dc-dc power converter system.

\section{Proposed Power Flow Scheme}

This section is intended to demonstrate the proposed structure of power flow in the DAB converter by using the variable magnetic element as well as PS modulation. Special emphasis is given to the improvements brought about by replacing the fixed inductor in a conventional DAB converter by a variable one.

\section{A. Control of Power Transfer Using VI}

As mentioned in the previous section, the value of the effective inductance in the converter can be varied by means of biasing the operation point on the $B(H)$ curve of the magnetic core, using a $\mathrm{dc}$ bias control current flowing through the control windings. For reference, Fig. 12(a) depicts the simulated inductance variation as a function of the bias control current, when varying the current from 0 to $1 \mathrm{~A}$ in steps of $0.1 \mathrm{~A}$. The inductance value is observed to decrease from a maximum value of $140 \mu \mathrm{H}$ at no saturation, to $60 \mu \mathrm{H}$ at full saturation, which is around $60 \%$ variation range. Fig. 12(b), in turn, shows the resulting variation in the power transfer in the DAB converter as a function of the dc bias control current, the power level varies from the nominal value of 500 to $2100 \mathrm{~W}$, which is nearly three times greater than the nominal. 


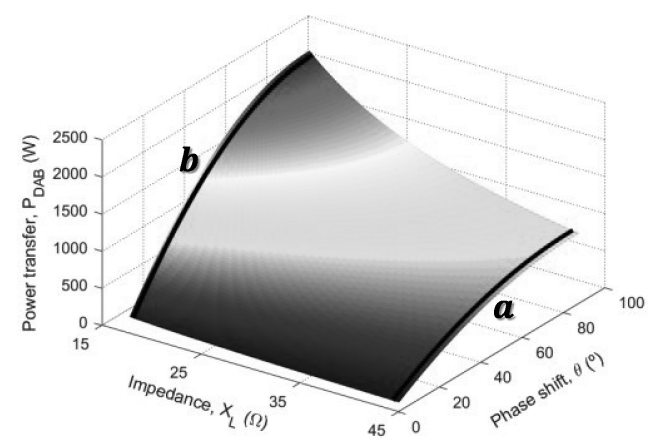

Fig. 13. Power transfer in a DAB converter as a function of inductance and PS.

This illustrates that the VI can serve as a control parameter for the power transfer in the converter.

Fig. 13 further adds a third dimension to depict the effect of PS as a control parameter, in combination with the inductance variation. As it can be observed, at a certain inductance value, as the PS increases, the power transfer in the DAB converter increases correspondingly, based on (1). However, this increase of the power becomes less significant as the inductance value increases.

This can be clarified by comparing the operation at the two different curves $\boldsymbol{a}$ and $\boldsymbol{b}$. At $0 \mathrm{~A}$ bias current, where $L$ is maximum, as the PS increases, the power transfer follows the curve $\boldsymbol{a}$. A relatively small variation in the power is possible. However, at $1 \mathrm{~A}$ bias, where $L$ is minimum, as the PS increases, the power transfer follows the curve $\boldsymbol{b}$, which shows more than double the variation of the power transfer compared to that at curve $\boldsymbol{a}$.

From another side, the figure also defines the limitations imposed by the PS control parameter on the variable inductance one. The figure clarifies that for higher PS, the decrease of the inductance can achieve larger output power compared to lower PS. In other words, the effect of the bias control current of the VI is increased as the PS between bridges increases toward the maximum. Therefore, selecting the inductor value for a specific operation range is a critical process in a $\mathrm{DAB}$ converter design.

\section{B. Linearization of the Control Function}

As explained in the previous section, the power transfer of a DAB converter is regulated by (1), as a function of several parameters. Fig. 14(a) shows the power transfer characteristics in the DAB converter as a function of the PS. It can be observed that the power transfer increase is approximately linear with the increase of PS until a certain limit, after which the rate of increase of power drops following a sinusoidal waveform until reaching the maximum power transfer at $90^{\circ} \mathrm{PS}$ angle. This characteristic operation is intrinsic to the PS DAB converter, and thus, to control the power transfer in the converter, the PS is used as the primary control parameter. By manipulating (1), the following expression is obtained:

$$
\theta=\frac{\pi}{2}\left(1 \pm \sqrt{1-\frac{4 P_{\mathrm{DAB}} X_{L} r_{t}}{\pi V_{\mathrm{in}} V_{\mathrm{out}}}}\right)
$$
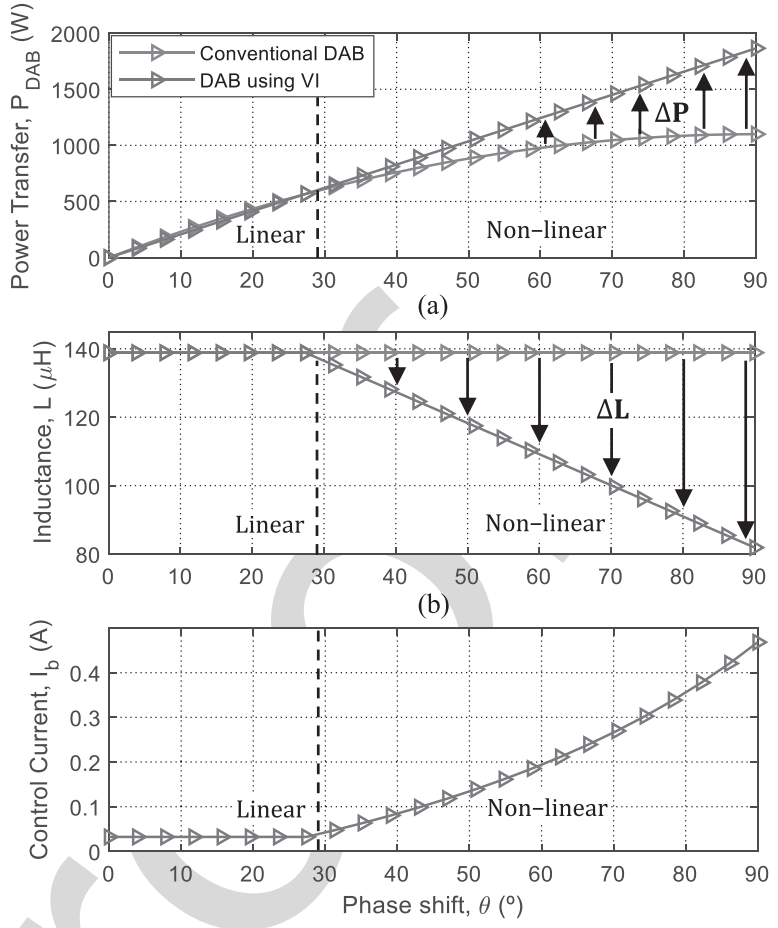

(c)

Fig. 14. Linearization of power transfer characteristics in the proposed DAB converter using VI. (a) Power transfer, (b) inductance, and (c) bias control current command as a function of PS angle.

which explicitly defines the PS angle $\theta$ as a function of the output power $P_{\mathrm{DAB}}$. As it can be observed, (5) presents a nonlinear equation, introducing difficulty in implementation and excessive processing time, as well as affecting the dynamic performance of the digital controller. Therefore, it is promising to use the inductance as an extra control parameter to allow the linearization of the power transfer function, and thus achieving a linear control function, the merits of which will be discussed as follows.

Fig. 14(a) illustrates the nonlinear power transfer curve of the conventional DAB converter, compared to the linearized one achieved by making use of the variation in the inductance. As it can be observed, the linear power transfer curve coincides, approximately, with the nonlinear curve in the range of $\theta=$ $0^{\circ}-30^{\circ}$, which is illustrated on the plots as the linear region.

Therefore, the phase-to-power relationship of the linear power curve can be defined by the slope of the straight line. Consequently, by substituting the values of the power at $0^{\circ}$ and $30^{\circ}$ in (1), the linear power-to-phase relationship can be defined by

$$
\theta_{\text {lin }}=\frac{X_{L} \cdot I_{\mathrm{out}} \cdot r_{t}}{V_{\mathrm{in}}(5 / 6)}
$$

The inductance is constant during the linear region, as demonstrated in Fig. 14(b), while in order to increase the power transfer by $\Delta P$ and keep the linearity of the function, the inductance is reduced by $\Delta L$, as illustrated in the figure. The inductance is computed as a function of the power transfer and PS angle, as 


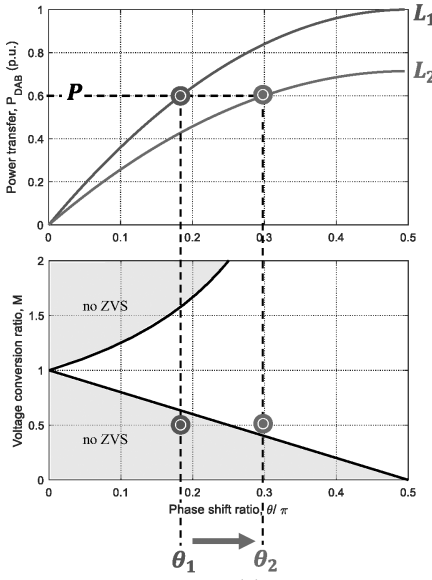

(a)

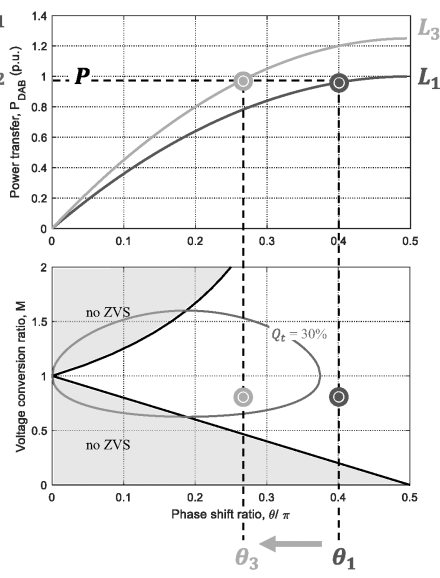

(b)
Fig. 15. Power transfer in the DAB converter (top plot), and the corresponding voltage conversion ratio (bottom plot), showing two operation conditions: (a) light load and (b) heavy load.

shown in the following, which is derived from (1):

$$
L=\frac{V_{\mathrm{in}} V_{\mathrm{out}}}{2 \pi f \cdot r_{t} \cdot P_{\mathrm{DAB}}} \theta\left(1-\frac{\theta}{\pi}\right) .
$$

In order to achieve the calculated inductance variation in the magnetic device, the bias current is increased, as shown in Fig. 14(c).

\section{Combination of Inductance Variation With Modulation Schemes to Improve the Converter Efficiency}

At light loading condition, i.e., $V_{\text {out }} \ll r_{t} V_{\text {in }}$, two issues take place which contribute significantly to increase the power losses and thus to reduce the efficiency in this operation region. First, the loss of ZVS due to insufficient energy required to charge and discharge the MOSFET capacitors causing elevated switching losses [1]. And second, relatively small PS values interfere with the deadtime range causing a phenomenon called "voltage polarity reversal" [5], [36] and limiting the minimum power transfer. Those two issues cause an increase in the reactive power component and consequently, reduce the efficiency at this load range. In addition, at high loading condition, i.e., $V_{\text {out }} \gg r_{t} V_{\text {in }}$, relatively large circulating currents take place, causing an increase in conduction losses and elevating reactive power losses [4], [22].

In this context, varying the inductance to have a relatively higher value at light load allows for increasing the PS and maintaining ZVS for a wider range, as well as avoiding the interference with the deadtime. And, on the other hand, decreasing the inductance value at heavy load allows for reducing the circulating currents.

To demonstrate the idea, Fig. 15 explains the two conditions of low and high loading. The figure shows the power transfer in the DAB represented in per unit (p.u.) values, and the corresponding voltage conversion ratio. The different boundaries of ZVS and circulating currents are indicated to clarify the sought operation zone. Fig. 15(a) shows that at a low loading, a small PS angle $\theta_{1}$ could lead the converter to operate in a no ZVS region. However,

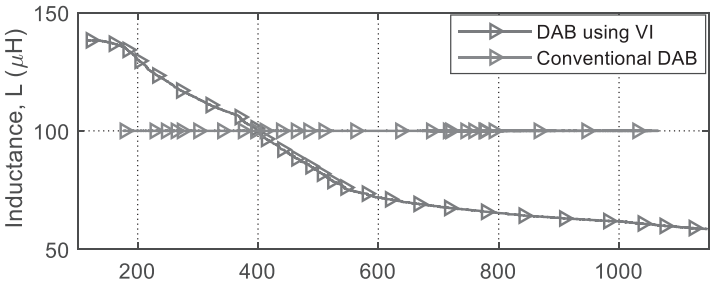

(a)

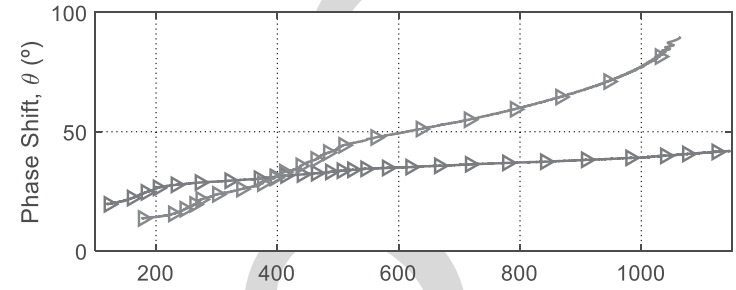

(b)

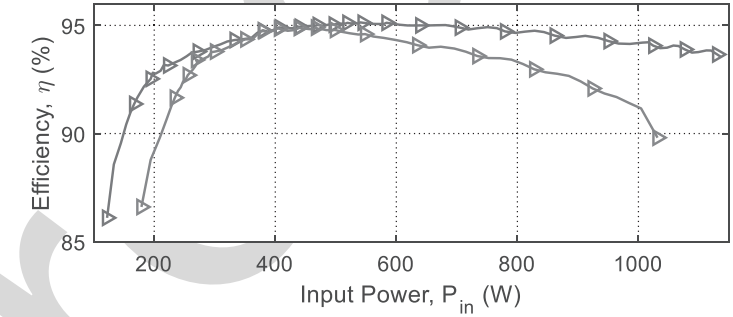

(c)

Fig. 16. Efficiency of DAB converter using VI. (a) Inductance value, (b) PS, and (c) converter efficiency as a function of output power transfer.

to achieve the same power level $P$, the inductance value can be increased from $L_{1}$ to $L_{2}$. This allows increasing the PS angle to $\theta_{2}$ to be able to operate under ZVS once again. In the same manner, Fig. 15(b) shows that at a high loading, a large PS angle $\theta_{1}$ could lead the converter to operate under high circulating currents, exceeding the required percentage reactive currents boundary. However, to achieve the same power level $P$, the inductance value can be decreased from $L_{1}$ to $L_{3}$. This allows decreasing the PS angle to $\theta_{3}$ to be able to operate within the reactive boundaries once again.

Using the simulation model, this analysis is verified by comparing the efficiency of the full DAB converter using the conventional constant inductance against that using the variable inductance. Fig. 16 illustrates the simulation results. Fig. 16(a) compares the inductance in a conventional DAB converter to that of the proposed DAB using VI. While the conventional inductance is observed to be constant at $100 \mu \mathrm{H}$, the VI changes its value from the maximum of $144 \mu \mathrm{H}$ to the minimum of around $50 \mu \mathrm{H}$. The PS angle is calculated in the simulation model by substituting $L$ in (5), as illustrated in Fig. 16(b). It can be observed that, in the case of the constant inductance, the PS varies between the minimum and maximum values to adjust the power transfer. The small PS angles applied at light loading implicate a loss in the ZVS and interference with the deadtime. While at a high loading condition, the large PS angles elevate the reactive power losses. On the other hand, observing the PS curve in the case of using variable inductance, it can be observed that the variation is limited to a small range, $20^{\circ}<\theta<50^{\circ}$. This 


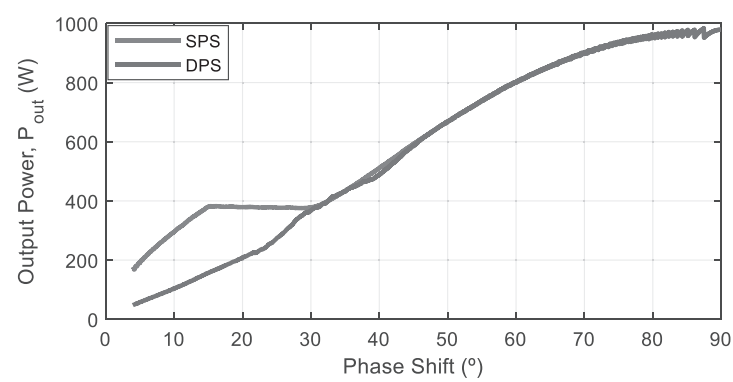

Fig. 17. Output power delivered by the DAB converter as a function of the PS angle, comparing SPS to DPS modulation strategies.

enhances the efficiency under the latter conditions of operation, as observed in Fig. 16(c).

Another feature is that the variation of the inductance can allow for achieving ZVS over the full operation range if combined with a modulation scheme such as the dual-PS (DPS) [37], [4]. This can be observed in Fig. 17, which compares the power flow in the DAB converter for the cases of SPS and DPS modulations.

More complicated modulation schemes are studied in some literature works [3], [6], [7], which aim at achieving ZVS over the full operation range of the converter. An example of those strategies is the triple-PS (TPS) [8]. Although the TPS is reported to provide the widest ZVS operation [8], it is also reported to pose more variables to the control function. This implies a more complicated implementation of the control calculations and requires higher processor capabilities [9]. In this manner, the use of VI can provide a good compromise between complexity and, on the other hand, the ease and time of implementation of the control function.

Therefore, based on the previous analysis, the proposed model takes advantage of combining the DPS modulation scheme with the variation of inductance in order to extend the range of the ZVS to the full operation of the converter.

\section{Power Flow Schemes Based on the Operation Mode}

It is necessary when using several control parameters to decide the combination of those parameters in the system control function. In the case of the DAB converter, the objective is to control the power transferred between the input and output ports. Specific to this converter, the characteristics of the power flow impose some restrictions on the operation over wide load ranges, as it has been discussed in the previous sections. Therefore, two modes of operation can be applied to combine the inductance with the PS as control parameters.

In the first mode, Mode I, the inductance value is used as the primary control parameter. This is a simplified mode where the power transfer can be adjusted throughout the full operation range by means of the variation of the inductance between the maximum and the minimum values. This mode assumes that the load, which the converter will supply, has a known (or predictable) profile as a function of time, which is the case in some applications, for example, in steel rolling mills. In such a condition, also the bias control current, which is responsible for adjusting the inductance value, can be defined as a fixed profile

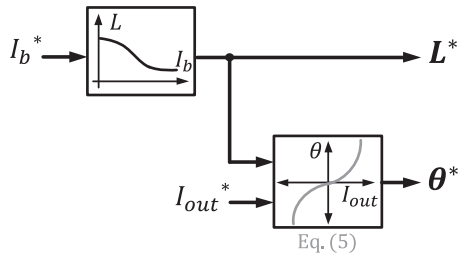

Fig. 18. Combination of variable inductance with PS to control the power flow in the DAB converter: Mode I.
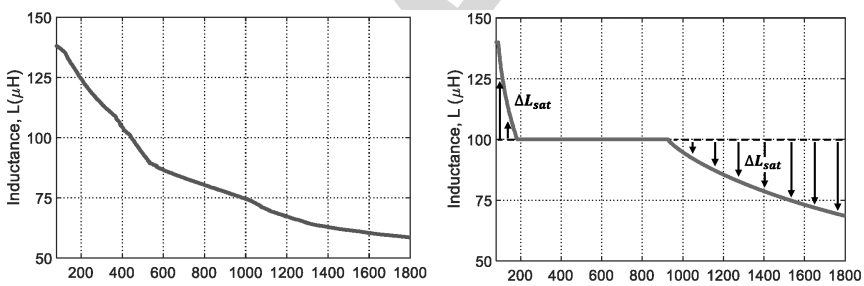

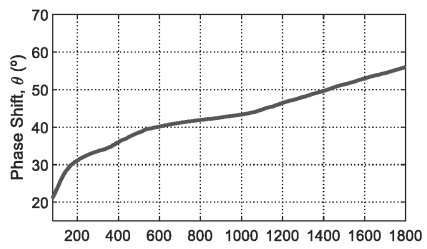

Output Power, $\mathrm{P}_{\text {out }}(\mathrm{W})$

(a)

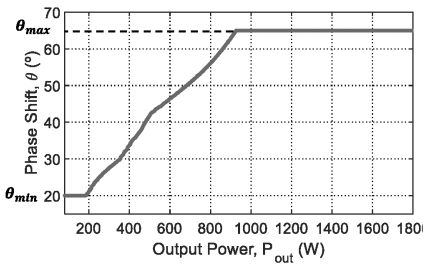

(b)
Fig. 19. Inductance (top) and PS angle (bottom) as a function of the output power in the DAB converter using VI. (a) Mode I. (b) Mode II.

as a function of time. This can allow disregarding the dc-dc converter required to control the bias current of the inductor and instead use a programmable source. Such simplification can be advantageous in some applications to reduce the number of sensors and unnecessary digital control computations. However, since the operation is based merely on open-loop calculations, some differences might occur between the required power and the provided one. This difference can be provided by the secondary control parameter, which is the PS angle between the converter bridges. To achieve a specific power, the required PS is, therefore, computed based on the current-to-phase relationship derived from (5) by replacing $P_{\mathrm{DAB}}$ by $V_{\text {out }} I_{\text {out }}$. Therefore, this mode of operation can be represented by the simplified block diagram in Fig. 18. The resulting waveforms are illustrated in Fig. 19(a). In this case, the load profile simulated is a linear ramp increasing as a function of time, which implies that the bias control current is a ramp proportional to the load profile. As $I_{b}$ is increased linearly, the inductance value is observed to reduce from maximum to minimum values, as it can be seen in the top plot. The variation of the PS angle, shown on the bottom plot, is observed to be bound in a small range, since it compensates the power difference between the designed and the required inductance values at each operation point.

If the inductor is well designed for the application, then, besides the simplicity of implementation, this mode will achieve two advantages. First, the phase-to-current relationship is always in the linear region, as it was mentioned in the previous section. And second, the value of the PS angle, compared to the conventional DAB, is higher at light loads to keep ZVS, and lower at 


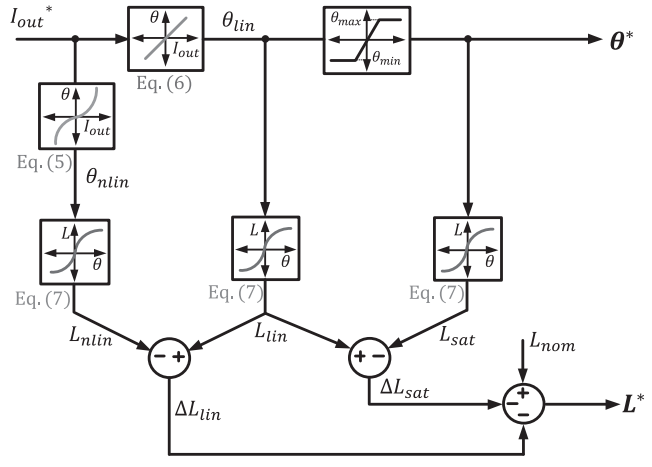

Fig. 20. Combination of variable inductance with PS to control the power flow in the DAB converter: Mode II.

heavy loads to reduce circulating currents. A better efficiency can thus be achieved over a wider load range.

On the other hand, in the second mode, Mode II, the PS angle is considered as the primary control parameter. This is more generic where the power transfer in the DAB converter is adjusted by means of the PS angle, while the inductance is used to achieve some enhancements to the power flow characteristics. This mode assumes that the load can vary in wide and unpredictable profiles, for example, in grid-tied applications, such as the SST-based microgrid paradigm under study, where the system exhibits disturbances of the demanded power and is susceptible to fault conditions. In such a condition, the reference PS angle $\theta_{l \text { in }}$ is calculated as a function of the demanded output current, $I_{\text {out }}^{*}$. This is done by using the linear current-to-phase relationship, previously expressed in (6), to keep the simplicity in digital implementation. The nonlinearity is thus compensated by the inductance variation. For this, the required variation of the inductance $\Delta L_{l i n}$ value is computed by using the phase-toinductance relationship in (7). Meanwhile, the reference of the PS angle is always saturated to a minimum and a maximum value $\theta_{\min }$ and $\theta_{\max }$, respectively, whether to the limits of ZVS and reactive currents or during transient events.

The minimum PS limit is defined by the deadtime and the minimum phase angle that achieves ZVS [35]. In this case, since the proposed model applies DPS to achieve ZVS at light load, then the minimum PS is simply the angle corresponding to the deadtime $1 \mu \mathrm{s}$, which corresponds to $18^{\circ}$ at $50 \mathrm{kHz}$ switching frequency. And, the maximum PS is $65^{\circ}$. Previous works [21] in the literature have limited the PS angle to $65^{\circ}$, due to the nonlinearity of the phase-power function, as it can be recalled from Fig. 14(a), where it can be observed that after $65^{\circ}$, the rate of increase of power drops. The amount of saturation in the PS angle is, hence, compensated by the inductance variation $\Delta L_{\text {sat }}$, which is computed by using (7). Fig. 20 shows a block diagram of the operation in Mode II. Also, the resulting waveforms are illustrated in Fig. 19(b), which when compared to Fig. 19(a), it can be observed that the PS is, similarly, limited by $\theta_{\min }$ and $\theta_{\max }$, and has a linear relationship as a function of current. Compared to Mode I, this mode involves more computations. However, it provides the two formerly mentioned advantages keeping a linear relation between the PS and the current as well

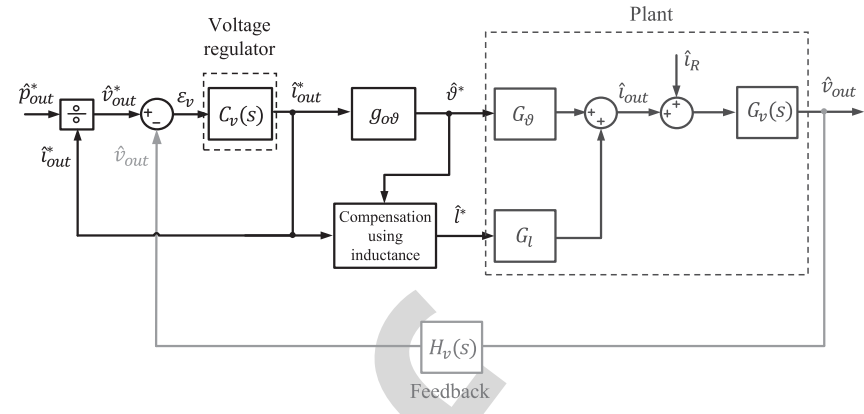

Fig. 21. Closed-loop control of DAB converter using inductance and PS as two control parameters.
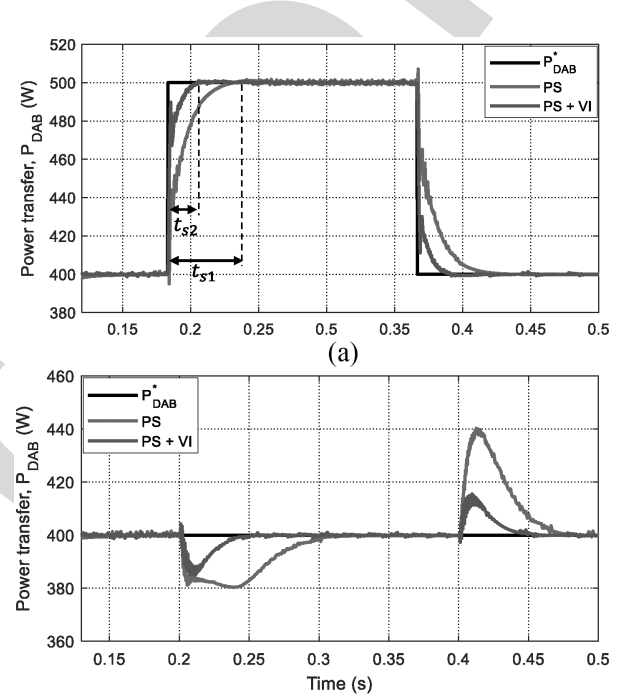

(b)

Fig. 22. Dynamics of PI controller comparing the control using the PS to that combining PS and VI.

as enhancing the efficiency over a wide load range. And, furthermore, making use of this combination of control parameters to design a closed-loop control for the system could allow to address unexpected system transients or disturbance events in an improved manner, as it will be demonstrated in the following section.

\section{E. Closed-Loop Control}

The closed-loop structure is based on the combination of the variable inductance and the PS as two control parameters. The structure is based on Mode II being the generalized operation mode, as shown in Fig. 21. The phase-current and inductancephase relationships are implemented based on (1). The dynamic performance of the controller is tested by introducing a step in the reference power. The step response of the control combining PS and VI is compared against that using PS only. Fig. 22 shows that the time response is smaller in case of adding the VI as a control parameter. This is due to the use of VI to compensate the saturation and linearization of the phase-to-current relationship as explained in the previous sections. Referring to the previous analysis, emphasis can be given to the advantages of using these modes of operation compared to the conventional DAB 


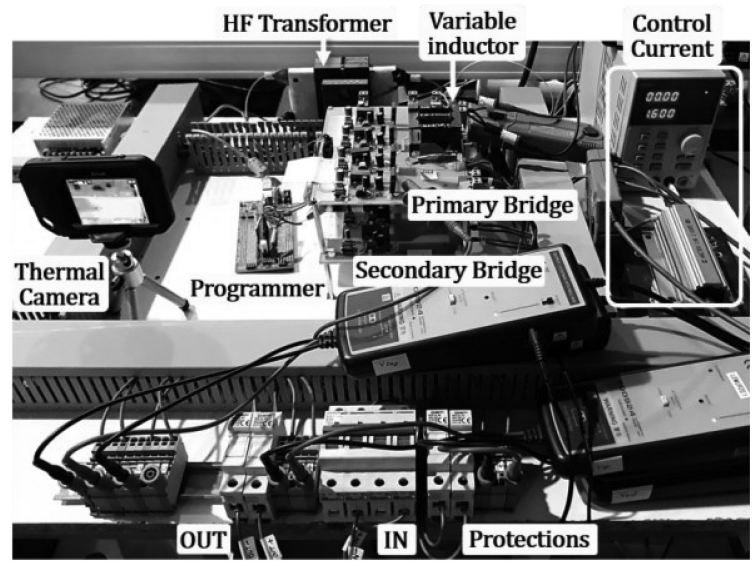

Fig. 23. Experimental setup.

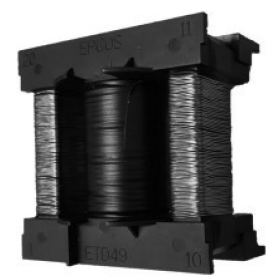

Fig. 24. Variable inductor prototype.

converter. First, the PI controller is tuned based on the linear phase-to-current relationship, which simplifies the mathematical operations and thus, decreases the processing time of the implemented control function. Second, the PS limitation does not affect the minimum power that can be transferred by the converter, since the inductance value is increased to compensate this. Third, circulating current can be significantly reduced due to the reduction of the PS angle especially at higher power, which is compensated by the decrease of the inductance value. And finally, the ZVS at light loads could be maintained by allowing the storage of enough energy in the inductor to charge and discharge the switches' capacitors.

\section{EXPERIMENTAL RESULTS}

\section{A. Experimental Platform}

A test platform has been developed to validate the electromagnetic model and the system analysis. The platform consists of a power stage and a programmer stage. The power stage includes the DAB converter as well as the VI. The DAB is composed of two full-bridge converters implemented using SiC MOSFETs. Each bridge is implemented on a separate PCB. The PCBs for the primary and secondary bridges are stacked on top of each other, with the VI prototype mounted on the primary bridge PCB, as seen in Fig. 23. The specific details of the design and operation parameters are summarized in Table II. Also, Fig. 24 shows the developed VI prototype. To supply the control winding of the VI, a variable dc voltage source is used in series with a resistor to have a maximum of $1.5 \mathrm{~A}$ dc bias control current. Additionally, a film capacitor is connected in parallel with the source terminals
TABLE II

SPECIFICATIONS OF THE TEST SETUP

\begin{tabular}{|c|c|c|}
\hline \multicolumn{3}{|c|}{ Power Stage } \\
\hline \multirow{6}{*}{$\begin{array}{c}\text { DAB } \\
\text { Converter }\end{array}$} & Rated power & $2 \mathrm{~kW}$ \\
\hline & Input voltage & $200 \mathrm{~V}$ \\
\hline & Output voltage & $250 \mathrm{~V}$ \\
\hline & Peak inductor current & $15 \mathrm{~A}$ \\
\hline & Switching frequency & $50 \mathrm{kHz}$ \\
\hline & Deadtime & $1 \mu \mathrm{s}$ \\
\hline \multirow{6}{*}{$\begin{array}{l}\text { Variable } \\
\text { Inductor }\end{array}$} & Core shape and size & ETD49/25/16 \\
\hline & Magnetic material & N87 \\
\hline & Inductance variation range & $144 \mu \mathrm{H}-50 \mu \mathrm{H}$ \\
\hline & DC voltage source & $0-30 \mathrm{~V}$ \\
\hline & Current limiting resistor & $10 \Omega$ \\
\hline & Peak DC control current & $3 \mathrm{~A}$ \\
\hline \multicolumn{3}{|c|}{ Programmer Stage } \\
\hline Hardware & $\mathrm{C2000}^{\mathrm{TM}}$ Real-time MCU & TMS320F28335 [29] \\
\hline Software & $\begin{array}{c}\text { Integrated development } \\
\text { environment }\end{array}$ & Code Composer Studio [30] \\
\hline
\end{tabular}

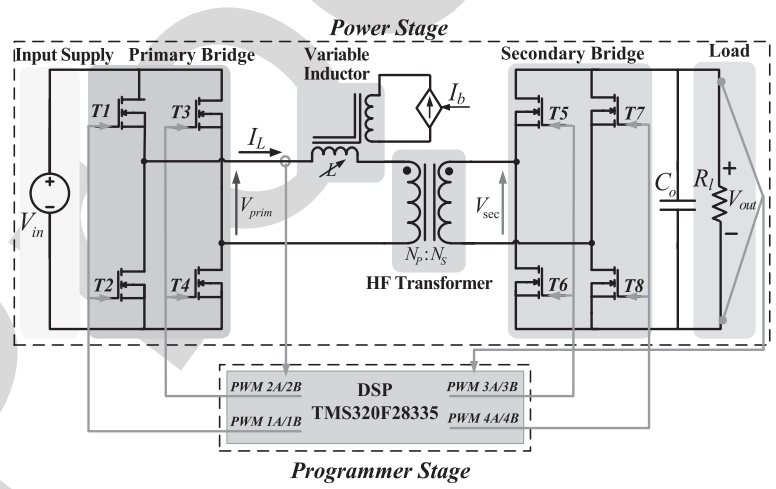

Fig. 25. Schematic of experimental setup.

to ensure that the voltage, and consequently the dc current, will be as smooth as possible.

The HF isolation is achieved by an HF planar transformer. The transformer ratings as well as the measured model parameters have been already detailed in Section III. The tests carried out consider the DAB converter feeding a resistive load. The load is connected to the output dc-link of the DAB converter.

On the other hand, the digital control stage consists of a TMS320F28335 controlCARD from Texas Instruments, which is a C2000 32-bit microcontroller unit (MCU) [29]. The controlCARD is mounted on a basis peripheral board for hardware development. The software platform used is the Code Composer Studio (CCS) from Texas Instruments [30]. The programmed code is configured to realize two main tasks-providing the switch modulation and processing the feedback measurements.

The hardware connections of the two stages, in addition to the firing pulses, are illustrated in Fig. 25.

\section{B. Impact of Inductance on Power Transfer in DAB Converter}

The inductance value is varied by means of adjusting the dc bias control current $I_{b}$. As the control current increases, the magnetic core will approach saturation. The permeability of the magnetic material will thus decrease, causing an increase in the reluctance and eventually, a decrease in the value of 


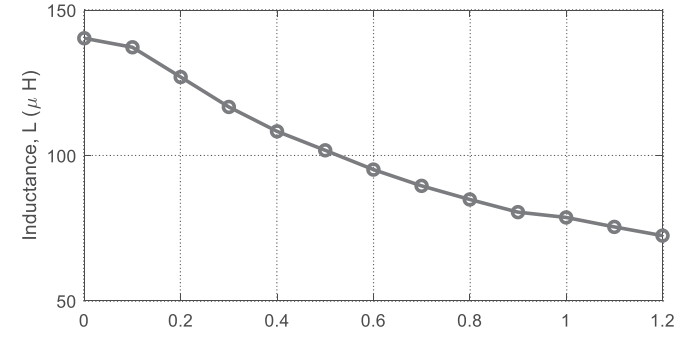

(a)

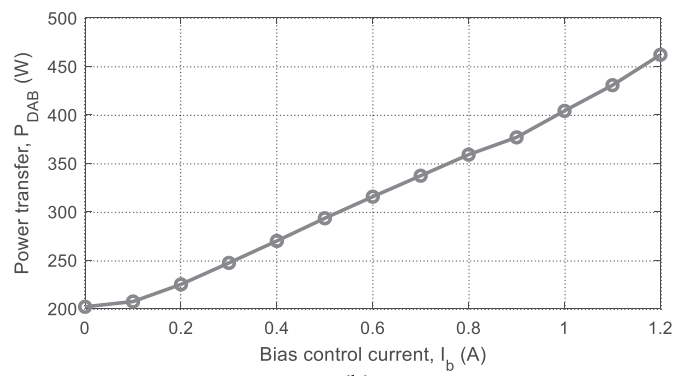

(b)

Fig. 26. Experimental results. Effect of inductance variation on power transfer in the DAB converter under buck operation mode. (a) Measured inductance and (b) power flow as a function of the bias control current.

the inductance measured from the main winding terminals. The decrease in the value of the inductance, in its turn, results in a decrease of the inductor impedance under a constant switching frequency of the converter, governed by (1). Specific to the DAB converter, the operation mode can vary depending on the levels of the input and the output voltage. The voltage conversion ratio $M$ has been previously defined to differentiate those modes of operation, such that $M=1$ refers to $V_{\text {in }}=V_{\text {out }} / r_{t}$, while $M<1$ refers to $V_{\text {in }}>V_{\text {out }} / r_{t}$ which is identified as the buck mode, and $M>1$ refers to $V_{\text {in }}<V_{\text {out }} / r_{t}$ which is identified as the boost mode.

Two experiments are thus carried out to identify the effect of the inductance variation on the power transfer in both latter conditions of operation. Based on this configuration, the power is transferred from the primary bridge to the secondary one, i.e., to the load, while the PS is kept constant at the maximum value of $\theta=90^{\circ}$. The dc bias current supplying the VI control windings is increased from 0 to $1.2 \mathrm{~A}$ in steps of $0.1 \mathrm{~A}$. The power transfer can, hence, be computed using the measurements of the voltage across the VI main winding, and the current flowing through it. The inductance value is, similarly, computed from the measured voltage and current, by using the rms of the first harmonic component of the waveforms [26].

Fig. 26 illustrates the measured inductance and the power transfer in the DAB as a function of the dc control current for the buck mode, along with the corresponding instantaneous voltage and current waveforms measured across the inductor main winding illustrated in Fig. 27. Meanwhile, the same experiment was repeated also for a higher loading condition, operating under the boost mode to reach a maximum power level of $1.8 \mathrm{~kW}$, as shown in Fig. 28. The peak inductor current is boosted from $5 \mathrm{~A}$ at no saturation to $15 \mathrm{~A}$ at full saturation of the magnetic core, as it can be observed in Fig. 29.

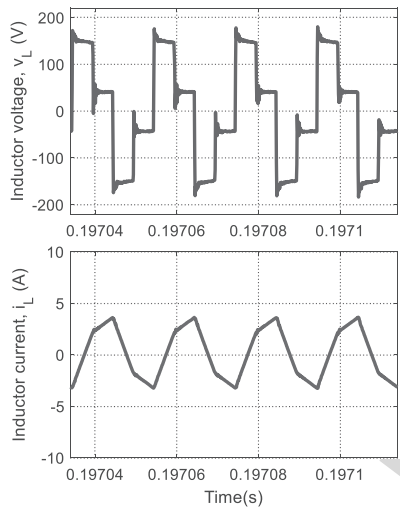

(a)

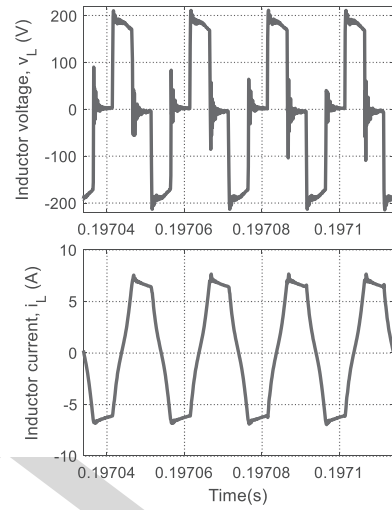

(b)
Fig. 27. Open-loop experimental results for DAB operating in buck mode the ac inductor voltage (top) and current (bottom) for three levels of dc bias control current: (a) $0 \mathrm{~A}$ and (b) $1.2 \mathrm{~A}$.

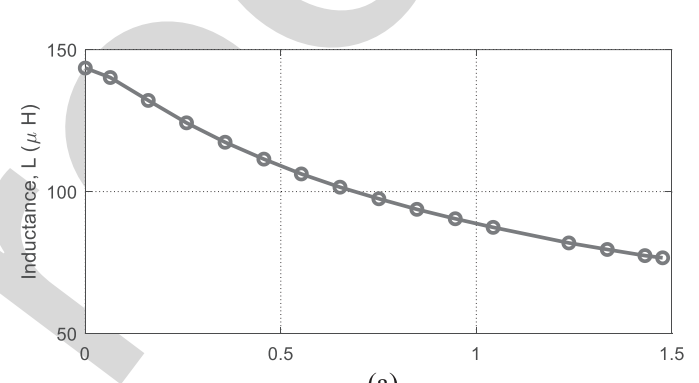

(a)

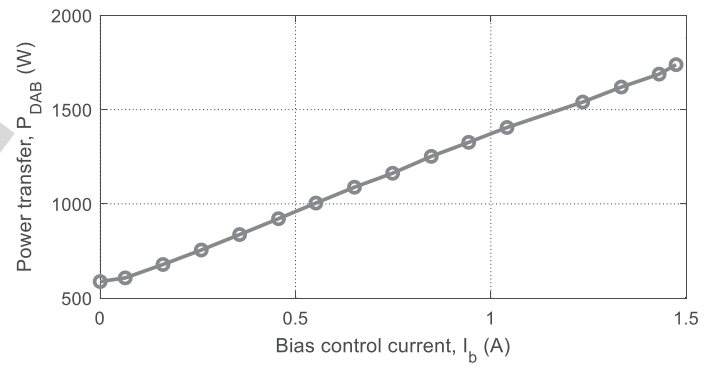

(b)

Fig. 28. Experimental results. Effect of inductance variation on power transfer in the DAB converter under boost operation mode. (a) Measured inductance and (b) power flow as a function of the bias control current.

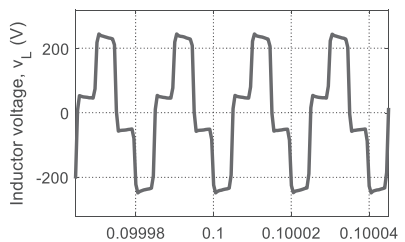

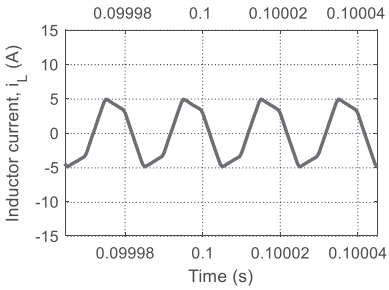

(a)
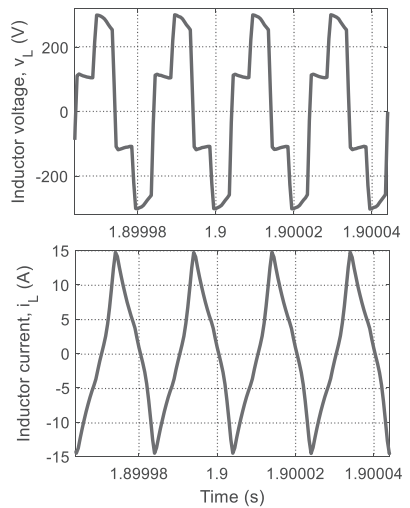

(b)
Fig. 29. Open-loop experimental results for DAB operating in boost mode. The ac inductor voltage (top) and current (bottom) for three levels of dc bias control current: (a) $0 \mathrm{~A}$ and (b) $1.5 \mathrm{~A}$. 


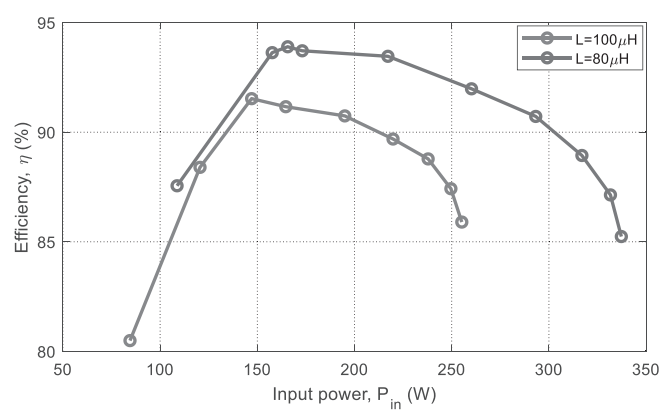

Fig. 30. Experimental results. Efficiency curves when using PS to control the power transfer in the DAB converter prototype.

\section{Efficiency Curves}

After analyzing the operation of the $\mathrm{DAB}$ converter prototype using the VI, the merits of using the variable inductance for improving the operation of the $\mathrm{DAB}$ converter are explored in this section. To achieve this, several measurements have been carried out for recording the efficiency curves of the full converter as a function of the power transferred. To conceive a comprehensive comparison, three operation scenarios are studied, as explained below.

1) PS Angle as the Primary Control Parameter: The first is the conventional scenario of controlling the power transfer in the DAB by means of varying the PS between the primary and the secondary bridges, while keeping the inductance value constant. The PS angle between the primary and secondary bridges is varied from $0^{\circ}$ to $90^{\circ}$ to transfer power from the input to the load, while the inductance value is kept constant at $100 \mu \mathrm{H}$.

For the experiments carried out herein, the DAB converter is operated in the buck mode, whereby the test conditions presented in the previous section are replicated. Fig. 30 illustrates the efficiency curves calculated for both experiments as a function of the input power. It can be observed that, for $80 \mu \mathrm{H}$ inductance, the efficiency value ranges from $85 \%$ to $94 \%$, while the peak value is shifted to $165 \mathrm{~W}$. Comparing both efficiency curves, it can be observed that using a lower inductance achieves a higher efficiency, especially as the level of power increases. This occurs at high loading condition, since reducing the inductance value allows transferring the same power with a smaller PS angle, which implies a smaller angle between the voltage and current applied to the transformer, and consequently, lower reactive power losses.

2) Inductance as the Primary Control Parameter: On the other hand, the second scenario studied is to control the power transfer in the DAB by means of variation of the inductance value through controlled saturation, as discussed previously, while in this case, the PS between the bridges is kept constant. Similar to the previous analysis, this experiment is repeated for two different values of the PS angle, $50^{\circ}$ and $90^{\circ}$. Fig. 31 illustrates the efficiency curves as a function of the input power for both values of PS angle. In this case, the efficiency curves are observed to be more flat, presenting smaller efficiency fluctuation between the maximum and minimum values experienced. As the PS angle is reduced from $90^{\circ}$ to $50^{\circ}$, the efficiency values are increased by

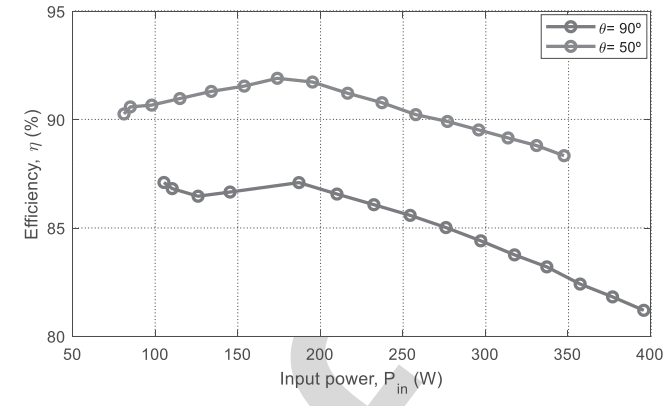

Fig. 31. Experimental results. Efficiency curves when using inductance to control the power transfer in the DAB converter prototype.

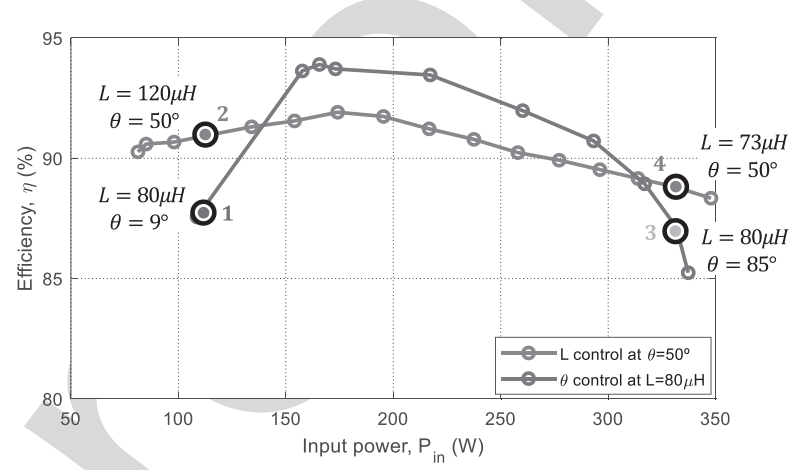

Fig. 32. Experimental results. Efficiency curves when combining inductance with PS to control the power transfer in the DAB converter prototype.

almost $6 \%$, in a nearly linear translation of the efficiency curve upward. This conclusion is, similarly, attributed to the reduction of the circulating currents and consequently, the reactive power losses, which eventually leads to higher efficiency.

3) Combining PS and Inductance as Control Parameters: Finally, both the previous scenarios are combined to compare the efficiency curves of the full DAB converter, with the aim of demonstrating the efficiency improvement accomplished by combining both parameters to control the power transfer. Fig. 32 illustrates two efficiency curves; one is measured at the operation scenario whereby the PS angle is used as the control parameter while keeping the inductance constant at $80 \mu \mathrm{H}$. The other curve is measured at the operation scenario whereby the inductance is used as the control parameter while keeping the PS constant at $50^{\circ}$. Those are the curves which presented the highest efficiency during the previous efficiency comparative tests.

Within the conditions of operation of the experiment carried out, at the specific input voltage of $100 \mathrm{~V}$ and load value of $50 \Omega$, the nominal value of the power transfer is approximately $200 \mathrm{~W}$, which can be calculated by (1). Around this value, it is apparent that using the PS as the control parameter achieves higher efficiencies. On the other hand, for low and high loading conditions, the use of the VI achieves higher efficiency values. To clarify this observation, two points are selected for comparison under each of the operation conditions such that the input power level is kept constant, and thereby, a constant voltage conversion ratio $M$. Hence, points $\mathbf{1}$ and $\mathbf{2}$ are compared for the low loading operation condition, while points $\mathbf{3}$ and $\mathbf{4}$ are compared for the high loading condition. 


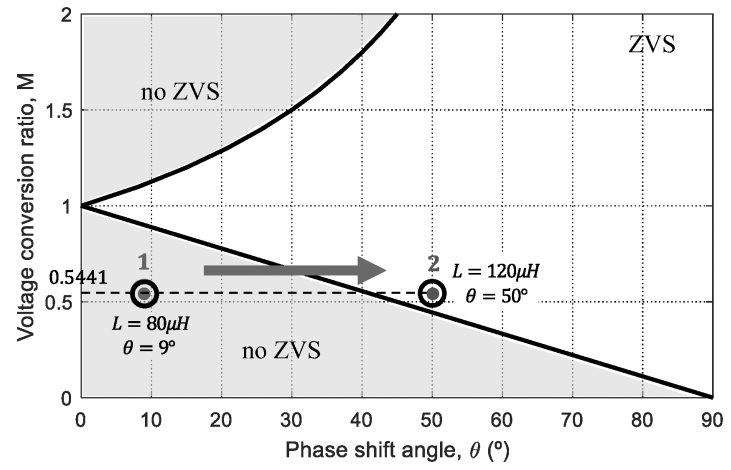

Fig. 33. Experimental results. The voltage conversion ratio $M$ as a function of the PS angle $\theta$ in degrees, illustrating the boundaries of ZVS in the DAB converter, for comparing the experimental operation points $\mathbf{1}$ and $\mathbf{2}$.
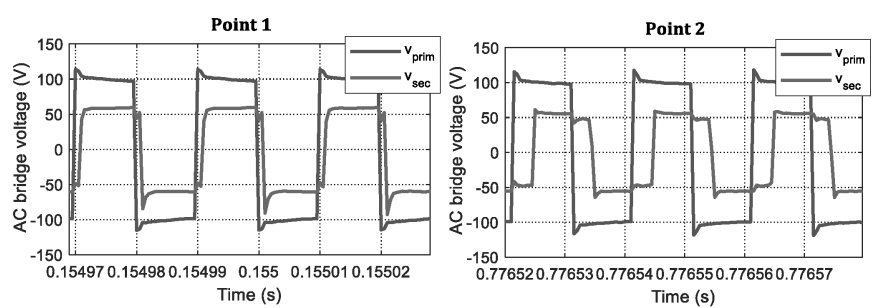

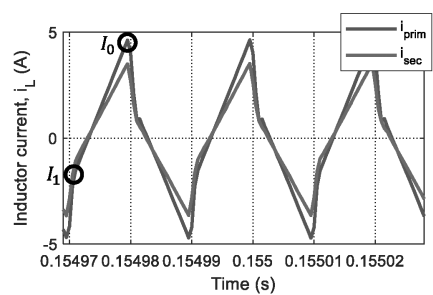

(a)

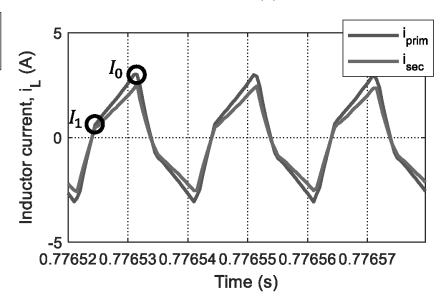

(b)

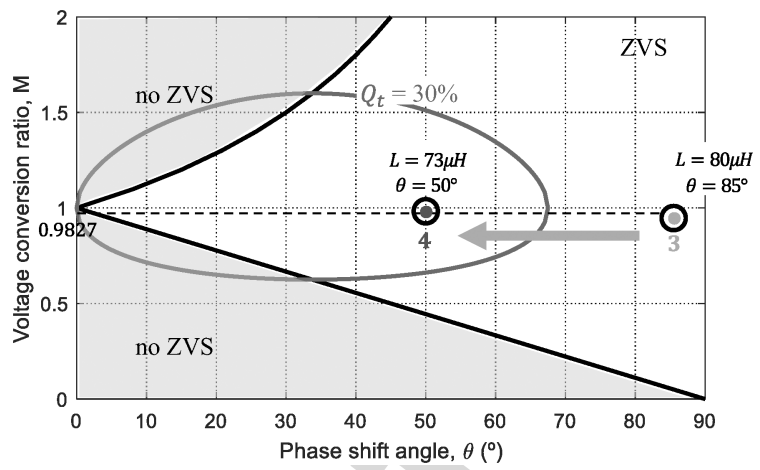

Fig. 35. Experimental results. The voltage conversion ratio $M$ as a function of the PS angle $\theta$ in degrees, illustrating the boundaries of ZVS in the DAB converter, for comparing the experimental operation points $\mathbf{3}$ and $\mathbf{4}$.
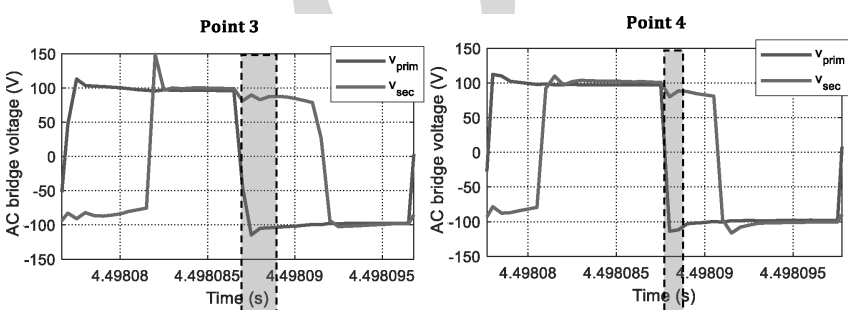

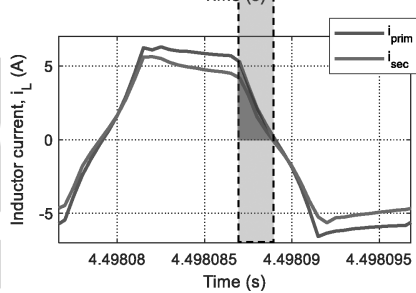

(a)

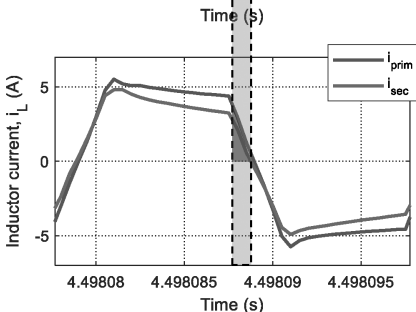

(b)

Fig. 34. Experimental results. The measured ac primary and secondary bridge voltages (top) and the corresponding inductor current measured at the primary and secondary (bottom), for comparing the experimental operation at: (a) point $\mathbf{1}$ and (b) point 2.

To compare the points $\mathbf{1}$ and $\mathbf{2}$, Fig. 33 is generated for $M$ as a function of $\theta$, in degrees, while illustrating the ZVS boundaries. This analysis is an experimental application of the theoretical study performed previously in Section II, precisely recalling Fig. 15(a). It can be observed that point 1 presents an operation condition of the converter at no ZVS.

This explains the low efficiency obtained when comparing to point $\mathbf{2}$. Therefore, moving to point $\mathbf{2}$ can allow to bring the converter operation again into the ZVS region. This is due to the fact that increasing the inductance value from $80 \mu \mathrm{H}$, at point $\mathbf{1}$, to $120 \mu \mathrm{H}$, at point $\mathbf{2}$, implies that the PS is required to increase as well, from $90^{\circ}$ to $50^{\circ}$, to achieve the same power level. The higher PS implies a larger time to charge or discharge the parasitic capacitances of the MOSFETs and thus, achieve ZVS again.

Fig. 34 further clarifies the idea by illustrating the measured ac primary and secondary bridge voltages, and the corresponding ac current in the inductor measured at primary and secondary sides of the transformer. It can be observed that during the operation at point 2 , the values of the currents $I_{0}$ and $I_{1}$ are positive, which is the initial condition for achieving ZVS, as discussed previously
Fig. 36. Experimental results. The measured ac primary and secondary bridge voltages (top), and the corresponding inductor current measured at the primary and secondary (bottom), for comparing the experimental operation at: (a) point 3 and (b) point 4

in Section II. However, on the other hand, during the operation at point $\mathbf{1}$, the value of the current $I_{1}$ is negative, so the ZVS is not achieved in this case.

On the other hand, to compare points $\mathbf{3}$ and $\mathbf{4}$, Fig. 35 is generated, while additionally illustrating the boundaries for the reactive currents. It can be observed that point $\mathbf{3}$ presents an operation condition of the converter at high circulating currents, which exceeds the set boundary. This explains the low efficiency obtained when comparing to point 4 . Therefore, when moving to point 4 , as the inductance value is decreased from 80 to $73 \mu \mathrm{H}$, the PS is required to decrease as well, from $85^{\circ}$ at point 3 to $50^{\circ}$ at point 4 , to achieve the same power level. The lower PS angle implies a lower angle between the voltage and current applied to the transformer, thereby lower circulating currents, which brings the converter operation again within the boundaries of the reactive currents.

Fig. 36 further clarifies the idea by illustrating that the measured ac waveforms are similar to the previous case. The reactive current is marked by the red shaded areas on the plots. It can be observed that during the operation at point $\mathbf{3}$, the reactive current is higher than that occurring during the operation point 4. 
To conclude this discussion, it can be conceived that combining the PS angle with the inductance as two control parameters of the power flow in the $\mathrm{DAB}$ converter results in boosting the efficiency along the entire operation range.

\section{CONCLUSION}

A DAB converter using VI has been proposed. An electromagnetic model has been developed which describes the behavior of a VI using the reluctance equivalent circuit and including the device losses. Also, the VI was simulated within a DAB converter in a circuit-based time-domain simulation. Using these models, a new degree of freedom was explored for the control of power transfer in the DAB converter using VI, as well as studying the operational margins for combining both PS control with the variable inductance one. Moreover, the analysis opened several aspects for the improvement of DAB converter operation using the VI solution, such as extending the limits of the power transfer in the converter, linearization of the control function, and boosting the converter efficiency over a wider load range. The experimental results clarified the effect of the inductance variation on the power transfer in a DAB converter under maximum PS, and the overall efficiency of the proposed DAB exceeded the conventional converter by around 5\% at light and heavy loading conditions.

While it is true that the controlled saturation of the magnetic core allows handling a higher power with the same core size [18], however, at this point in the investigation it cannot be postulated, on an undisputed technical basis, that this also implies a reduction in the final size of the entire converter. To arrive at such a conclusion, the study herein can be extended to consider the extra size of the electronic components added for controlling the VI. Given the fact that these components should be rated for very small power, i.e., $<10 \mathrm{~W}$, therefore, it is quite feasible to optimize the size of the extra control converter [39]-[41]. It is also worth to note that scaling up the converter to higher power levels is not limited by the VI. The device can be designed for a higher power level by utilizing a core material which presents higher values of magnetic flux saturation, such as iron powder [14], [42]. Additionally, the VI design can be modified to allow higher inductance variation ranges.

As a future development, the shares of the losses among the different converter components can be studied and demonstrated for the full operation range.

\section{REFERENCES}

[1] H. Fan and H. Li, "High-frequency transformer isolated bidirectional DCDC converter modules with high efficiency over wide load range for 20 kVA solid-state transformer," IEEE Trans. Power Electron., vol. 26, no. 12, pp. 3599-3608, Dec. 2011.

[2] A. Burgio, D. Menniti, M. Motta, A. Pinnarelli, N. Sorrentino, and P. Vizza, "A laboratory model of a dual active bridge DC-DC converter for a smart user network," in Proc. IEEE 15th Int. Conf. Environ. Elect. Eng., Jun. 2015, pp. 997-1002.

[3] G. Oggier, G. O. García, and A. R. Oliva, "Modulation strategy to operate the dual active bridge DC-DC converter under soft switching in the whole operating range," IEEE Trans. Power Electron., vol. 26, no. 4, pp. 1228-1236, Apr. 2011.

[4] H. Bai and C. Mi, "Eliminate reactive power and increase system efficiency of isolated bidirectional dual-active-bridge DC-DC converters using novel dual-phase-shift control," IEEE Trans. Power Electron., vol. 23,no. 6, pp. 2905-2914, Nov. 2008.
[5] Y. Xie, J. Sun, and J. S. Freudenberg, "Power flow characterization of a bidirectional galvanically isolated high-power dc-dc converter over a wide operating range," IEEE Trans. Power Electron., vol. 25, no. 1,pp. 54-66, Jan. 2010.

[6] F. Krismer and J. W. Kolar, "Efficiency-optimized high-current dual active bridge converter for automotive applications," IEEE Trans. Ind. Electron., vol. 59, no. 7, pp. 2745-2760, Jul. 2012.

[7] F. Krismer and J. W. Kolar, "Accurate small-signal model for the digital control of an automotive bidirectional dual active bridge," IEEE Trans. Power Electron., vol. 24, no. 12, pp. 2756-2768, Dec. 2009.

[8] C. Calderon, A. Barrado, A. Rodriguez, A. Lazaro, C. Fernandez, and P. Zumel, "Dual active bridge with triple phase shift by obtaining sof switching in all operating range," in Proc. IEEE Energy Convers. Congr Expo., 2017, pp. 1739-1744.

[9] J. Everts, "Closed-form solution for efficient ZVS modulation of DAB converters," IEEE Trans. Power Electron., vol. 32, no. 10, pp. 7561-7576, Oct. 2017

[10] S. Saeed, "Contributions to the design, operation and control of DAB bidirectional converters based on variable magnetic elements," $\mathrm{Ph} . \mathrm{D}$ dissertation, 2020

[11] P. Garcia, S. Saeed, A. Navarro-Rodríguez, J. García, and H. Schneider "Switching frequency optimization for a solid state transformer with energy storage capabilities," IEEE Trans. Ind. Appl., vol. 54, no. 6 pp. 6223-6233, Nov./Dec. 2018.

[12] Z. Qin, Y. Shen, P. C. Loh, H. Wang, and F. Blaabjerg, "A dual active bridge converter with an extended high-efficiency range by DC blocking capacitor voltage control,' IEEE Trans. Power Electron., vol. 33, no. 7, pp. 5949-5966, Jul. 2018.

[13] A. Rodríguez, A. Vásquez, D. G. Lamar, M. M. Hernando, and J. Sebastian, "Different purpose design strategies and techniques to improve the performance of a dual active bridge with phase-shift control,' in Proc. IEEE 15th Workshop Control Model. Power Electron., Jun. 2014 pp. $1-10$.

[14] M. S. Perdigão, M. Menke, A. R. Seidel, R. A. Pinto, and J. M. Alonso, "A review on variable inductors and variable transformers: Applications to lighting drivers," in Proc. IEEE Ind. Appl. Soc. Annu. Meeting, 2014, pp. $1-8$.

[15] E. Orietti, P. Mattavelli, G. Spiazzi, C. Adragna, and G. Gattavari, "Twophase interleaved LLC resonant converter with current controlled inductor," in Proc. Braz. Power Electron. Conf., 2009, pp. 298-304.

[16] J. M. Alonso, M. S. Perdigao, D. Gacio, L. Campa, and E. S. Saraiva "Magnetic control of DC-DC resonant converters provides constan frequency operation," Electron. Lett., vol. 46, no. 6, pp. 440-442, Mar. 2010.

[17] M. S. Perdigão, B. Baptista, H. Marques, E. S. Saraiva, and J. M. Alonso, "On the use of magnetic regulators in electronic ballasts," in Proc. 45th Int. Univ. Power Eng. Conf., 2010, pp. 1-6.

[18] M. S. Perdigão, J. Pedro F. Trovão, J. M. Alonso, and E. S. Saraiva, "Largesignal characterization of power inductors in EV bidirectional DC-DC converters focused on core size optimization," IEEE Trans. Ind. Electron., vol. 62, no. 5, pp. 3042-3051, May 2015.

[19] F. Krismer, S. Round, and J. W. Kolar, "Performance optimization of a high current dual active bridge with a wide operating voltage range," in Proc. 37th IEEE Power Electron. Spec. Conf., 2006, pp. 1-7.

[20] A. Rodríguez, J. Sebastian, D. G. Lamar, M. M. Hernando, and A. Vazquez, "An overall study of a dual active bridge for bidirectional DC/DC conversion," in Proc. IEEE Energy Convers. Congr. Expo., Sep. 2010, pp. 1129-1135.

[21] A. R. Alonso, "Análisis de arquitecturas multipuerto para la alimentación de sistemas electrónicos con múltiples flujos de energía," Ph.D. dissertation, Dept. Elect. Eng., Univ. Oviedo, Oviedo, Spain, 2015. [Online] Available: http://hdl.handle.net/10651/20286

[22] S. Saeed, "Design and construction of an isolated DC to DC switching converter for integration of energy storage systems in power electronic applications," M.S. thesis, Dept. Elect. Eng., Univ. Oviedo, Oviedo, Spain 2015. [Online]. Available: http://hdl.handle.net/10651/33257

[23] J. M. Alonso, G. Martínez, M. Perdigão, M. Cosetin, and R. N. do Prado, "Modeling magnetic devices using SPICE: Application to variable inductors," in Proc. IEEE Appl. Power Electron. Conf. Expo., 2016 pp. 1115-1122.

[24] S. Saeed, J. García, and R. Georgious, "Modeling of variable magnetic elements including hysteresis and eddy current losses," in Proc. IEEE Appl. Power Electron. Conf. Expo., 2018, pp. 1750-1755.

[25] D. Medini and S. Ben-Yaakov, "A current-controlled variable inductor for high frequency resonant power circuits," in Proc. IEEE Appl. Power Electron. Conf. Expo., 1994, pp. 219-225. 
[26] S. Saeed, R. Georgious, and J. Garcia, "Modeling of magnetic elements including losses-Application to variable inductor," Energies, vol. 13, no. 8, Apr. 2020, Art. no. 1865, doi: 10.3390/en13081865.

[27] ROHM. Datasheet of SiC MOSFET SCT2280KE. [Online]. Available: https://fscdn.rohm.com/en/products/databook/datasheet/discrete/sic/ mosfet/sct2280ke-e.pdf

[28] J. G. Tiemblo, "Investigations on reactive power and dead time compensation for a double active bridge with a planar transformer," Master's thesis, Univ. Oviedo, Oviedo, Spain, 2014. [Online]. Available: http://hdl.handle.net/10651/28158

[29] Texas Instruments ${ }^{\mathrm{TM}}$, TMS320F28335 digital signal controller. [Online]. Available: http://www.ti.com/lit/ds/symlink/tms320f28335.pdf?ts= 1588453618532

[30] Texas Instruments, Code Composer Studio integrated development environment for c2000 microcontrollers. [Online]. Available: http: //www.ti. $\mathrm{com} /$ tool/CCSTUDIO

[31] E. Cardelli, L. Fiorucci, and E. D. Torre, "Estimation of MnZn ferrite core losses in magnetic components at high frequency," IEEE Trans. Magn., vol. 37, no. 4, pp. 2366-2368, Jul. 2001.

[32] D. C. Jiles and D. L. Atherton, "Ferromagnetic hysteresis," IEEE Trans. Magn., vol. 19, no. 5, pp. 2183-2185, Sep. 1983.

[33] W. and Peter Reid, "Modelling and simulation of magnetic components in electric circuits," Ph.D. dissertation, Dept. Elect. Comput. Eng., Univ. Southampton, Southampton, U.K., Nov. 2001. [Online]. Available: https: //eprints.soton.ac.uk/368484/

[34] P. L. Dowell, "Effects of eddy currents in transformer windings," Proc. Inst. Elect. Eng., vol. 113, no. 8, pp. 1387-1394, Aug. 1966.

[35] A. Massarini and M. K. Kazimierczuk, "Self-capacitance of inductors," IEEE Trans. Power Electron., vol. 12, no. 4, pp. 671-676, Jul. 1997.

[36] B. Zhao, Q. Song, W. Liu, and Y. Sun, "Dead-time effect of the high frequency isolated bidirectional full-bridge dc-dc converter: Comprehensive theoretical analysis and experimental verification," IEEE Trans. Power Electron., vol. 29, no. 4, pp. 1667-1680, Apr. 2014

[37] M. Kim, M. Rosekeit, S. Sul, and R. W. A. A. De Doncker, "A dualphase-shift control strategy for dual-active-bridge DC-DC converter in wide voltage range," in Proc. 8th Int. Conf. Power Electron. - ECCE Asia, 2011, pp. 364-371.

[38] H. Fan and H. Li, "High-frequency transformer isolated bidirectional DCDC converter modules with high efficiency over wide load range for 20 kVA solid-state transformer," IEEE Trans. Power Electron., vol. 26, no. 12, pp. 3599-3608, Dec. 2011.

[39] M. W. Beraki, J. P. F. Trovão, M. S. Perdigão, and M. R. Dubois, "Variable inductor based bidirectional DC-DC converter for electric vehicles," IEEE Trans. Veh. Technol., vol. 66, no. 10, pp. 8764-8772, Oct. 2017.

[40] M. Beraki, M. Perdigao, F. Machado, and J.P. Trovão, "Auxiliary converter for variable inductor control in a DC-DC converter application," in Proc. 51st Int. Univ. Power Eng. Conf., 2016, pp. 1-6.

[41] J. M. Alonso, M. S. Perdigão, M. A. D. Costa, G. Martínez, and R. Osorio, "Analysis and experiments on a single-inductor half-bridge LED driver with magnetic control," IEEE Trans. Power Electron., vol. 32, no. 12, pp. 9179-9190, Dec. 2017.

[42] Y. Liu, H. A. Mantooth, J. C. Balda, and C. Farnell, "Realization of high-current variable AC filter inductors using silicon iron powder magnetic core," in Proc. IEEE Appl. Power Electron. Conf. Expo., 2017, pp. 855-860.

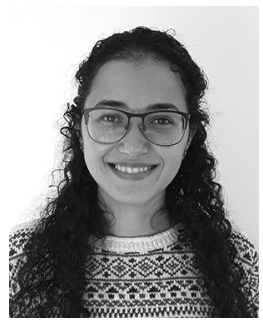

Sarah Saeed (Student Member, IEEE) received the B.Sc. degree in electrical engineering from Ain Shams University, Egypt, in 2013, and the M.Sc. degree in electrical energy conversion and power systems and the $\mathrm{Ph} . \mathrm{D}$. degree in energy and process control, both from the University of Oviedo, Spain, in 2015 and 2020, respectively.

She is a Postdoctoral Researcher with Lemur Research Group, University of Oviedo, Oviedo, Spain. She has coauthored five journal papers and 15 international conferences. Her research interests include the modeling and control of power electronic converters, design and construction of dc/dc converter topologies, integration of energy storage systems in power electronic applications, and nonlinear magnetics.

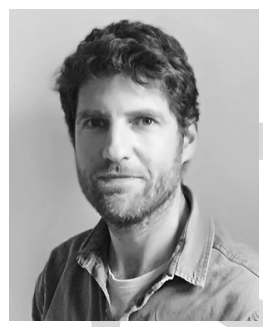

Jorge Garcia (Senior Member, IEEE) received the M.Sc. and Ph.D. degrees in electrical engineering from the University of Oviedo, Gijon, Spain, in 2000 and 2003, respectively.

In December 1999, he joined the Electrical and Electronic Engineering Department, University of Oviedo, where he is currently an Associate Professor. $\mathrm{He}$ is a Founding Member of the LEMUR Research Team. He has coauthored more than 50 journal papers and more than 100 international conference papers in power and industrial electronics. His research interests include analysis, modeling, and design of power electronics and control stages for industrial, grid support, and lighting applications.

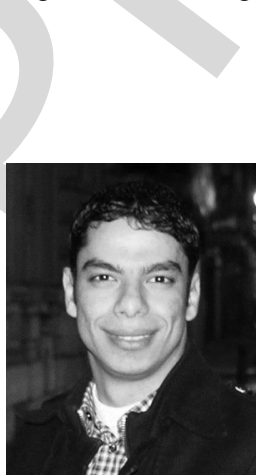

Ramy Georgious (Member, IEEE) received the B.Sc. degree in electrical engineering from Port Said University, Port Said, Egypt, in May 2010, and the M.Sc. degree in electrical energy conversion and power systems and the Ph.D. degree in electrical engineering from the University of Oviedo, Oviedo, Spain, in 2014 and 2018, respectively.

In July 2014, he joined LEMUR Research Team, University of Oviedo, Gijon, Spain. In 2015, he was a Visitor Researcher with Power Electronics, Machines, and Control Research Group, University of Nottingham, Nottingham, U.K. He is currently an Assistant Professor with the Department of Electrical, Computer, and Systems Engineering, University of Oviedo, and with the Electrical Engineering Department, Port Said University, and also a Director of the Hardware Research and Development Department, ENFASYS Engineering Company, Gijon, Spain. His research interests include energy storage systems, control systems, power electronic converters, microgrids, renewable energy, design, and simulation.
1081 1082 1083 1084 1085 1086 1087 1088 1089 1090 1091 1092 1093 1094 1095

1096 1097 1098 1099 1100 1101 1102 1103 1104 1105 1106 1107 1108 1109

1110 1111 1112 1113 1114 1115 1116 1117 1118 1119 1120 1121 1122 1123 1124 1125 1126 1127 